\title{
A new comprehensive set of elemental abundances in DLAs
}

\section{Data analysis and chemical variation studies $\star, \star \star$}

\author{
M. Dessauges-Zavadsky ${ }^{1}$, J. X. Prochaska ${ }^{2}$, S. D’Odorico ${ }^{3}$, F. Calura ${ }^{4}$, and F. Matteucci ${ }^{4,5}$
}

\author{
1 Observatoire de Genève, 51 Ch. des Maillettes, 1290 Sauverny, Switzerland \\ e-mail: miroslava.dessauges@obs.unige.ch \\ 2 UCO/Lick Observatory, University of California at Santa Cruz, Santa Cruz, CA 95064, USA \\ 3 European Southern Observatory, Karl-Schwarzschildstr. 2, 85748 Garching bei München, Germany \\ ${ }^{4}$ Dipartimento di Astronomia-Universitá di Trieste, via G. B. Tiepolo 11, 34131 Trieste, Italy \\ 5 INAF, Osservatorio Astronomico di Trieste, via G. B. Tiepolo 11, 34131 Trieste, Italy
}

Received 6 April 2005 / Accepted 5 August 2005

\section{ABSTRACT}

We present new, comprehensive sets of elemental abundances for seven damped Ly $\alpha$ systems (DLAs) in the redshift range $z_{\text {abs }}=1.8-2.5$. These were derived from UVES/VLT spectra combined with existing HIRES/Keck spectra. We detected 54 metal-line transitions, and obtained the column density measurements of 30 ions from 22 elements, - B, C, N, O, Mg, Al, Si, P, S, Cl, Ar, Ti, Cr, Mn, Fe, Co, Ni, Cu, Zn, Ge, As, Kr. Together with the four DLAs analyzed in Dessauges-Zavadsky et al. (2004, A\&A, 416, 79), we have a sample of eleven DLA galaxies with uniquely comprehensive and homogeneous abundance measurements. These observations allow one to study in detail the abundance patterns of a wide range of elements and the chemical variations in the interstellar medium of galaxies outside the Local Group. Comparing the gas-phase abundance ratios of these high redshift galaxies, we found that they show low rms dispersions, reaching only up 2-3 times the statistical errors for the majority of elements. This uniformity is remarkable given that the quasar sightlines cross gaseous regions with H I column densities spanning over one order of magnitude and metallicities ranging from 1/55 to 1/5 solar. The uniformity is also remarkable since DLAs are expected (and observed at low redshift) to be associated with a wide range of galaxy types. This implies the respective star formation histories seem to have conspired to yield one set of relative abundances. We examined the gas-phase abundance patterns of interstellar medium "clouds" within the DLA galaxies detected along the velocity profiles. By considering all the clouds of all the DLAs studied together, we observe a high dispersion in several abundance ratios, indicating that chemical variations seem to be more confined to individual clouds within the DLA galaxies than to integrated profiles. We found unambiguous correlations between $[\mathrm{Si} / \mathrm{Fe}],[\mathrm{S} / \mathrm{Fe}]$ and $[\mathrm{S} / \mathrm{Si}]$ versus $[\mathrm{Zn} / \mathrm{Fe}]$, and anti-correlations between $[\mathrm{Si} / \mathrm{Zn}]$ and $[\mathrm{S} / \mathrm{Zn}]$ versus $[\mathrm{Zn} / \mathrm{Fe}]$. These trends are primarily the result of differential dust depletion effects, which also explain the cloud abundance ratio dispersion. The signature of the nucleosynthesis enrichment contribution is observed in the $[\alpha / \mathrm{Fe}, \mathrm{Zn}]$ ratios at low dust depletion levels, $0 \leq[\mathrm{Zn} / \mathrm{Fe}] \leq 0.2$, and is characterized by an $\alpha$-enhancement in individual clouds. Quite surprisingly, however, while the $[\mathrm{Si} / \mathrm{Fe}]$ ratios are supersolar in clouds with low depletion level, the $[\mathrm{S} / \mathrm{Zn}]$ ratios remain almost solar, suggesting that [S/Zn] may not be a reliable tracer of nucleosynthesis enrichment. Analysis of the cloud-to-cloud chemical variations within seven individual DLA systems reveals that five of them show statistically significant variations, higher than 0.2 dex at more than $3 \sigma$, but only two DLAs show extreme variations. The sources of these variations are both the differential dust depletion and/or ionization effects; however, no evidence for variations due to different star formation histories could be highlighted. These observations place large constraints on the mixing timescales of protogalaxies and on scenarios of galaxy formation within the CDM hierarchical theory. Finally, we provide an astrophysical determination of the oscillator strength of the Ni II $\lambda 1317$ transition.

Key words. cosmology: observations - galaxies: quasars: absorption lines - galaxies: abundances - line: profiles

\section{Introduction}

One of the most exciting developments in observational cosmology over the last decade has been the ability to extend

\footnotetext{
* Based on UVES observations made with the European Southern Observatory VLT/Kueyen telescope, Paranal, Chile, collected during the programme ID No. 70.B-0258(A).

$\star \star$ Tables 2-8, Figs. 1-14, and Appendix A are only available in electronic form at http://www. edpsciences.org
}

studies of elemental abundances from the local Universe to high redshift. This is a fundamental step toward a better understanding of the formation and evolution of galaxies. It has been made possible by the studies of absorption line systems detected in optical spectra of quasars (QSOs), and specifically via the damped Ly $\alpha$ systems (DLA; e.g. Wolfe et al. 1986). These systems with H I column densities higher than $2 \times 10^{20} \mathrm{~cm}^{-2}$ dominate the neutral gas content of the Universe available for star formation and are, therefore, widely believed to be 
progenitors of present-day galaxies (Storrie-Lombardi \& Wolfe 2000, and references therein). The DLA systems provide the best opportunity to accurately measure the gas-phase chemical abundances of many elements for a variety of galactic systems spanning a wide redshift interval. These objects are by far the best laboratories for studying galaxies at high redshifts, in their early stages of evolution.

In Paper I (Dessauges-Zavadsky et al. 2004), we demonstrated the efficiency and power of this approach. Indeed, we obtained comprehensive sets of elemental abundances for four DLA systems, namely the abundance measurements of 15 elements - N, O, Mg, Al, Si, P, S, Cl, Ar, Ti, Cr, Mn, Fe, Ni, and $\mathrm{Zn}$ - and the column density measurements of up to 21 ions. This large chemical dataset allowed us to study each galaxy individually, while previously the DLA galaxy population had been analyzed as a whole. We also provided the first constraints on their star formation history, age, and star formation rate through a detailed comparison with a grid of chemical evolution models for spiral and dwarf irregular galaxies by Calura et al. (2003). These exciting results encouraged us to further extend our sample. We obtained high quality spectra of seven additional DLAs, bringing the full sample of DLA galaxies to eleven systems.

Three main sources influence the chemical gas-phase abundance composition of these high redshift galaxies: (i) the star formation history (SFH) through its implications for nucleosynthesis processes; (ii) differential dust depletion effects, since part of elements may be removed from the gas to the solid phase (Savage \& Sembach 1996); and (iii) photoionization effects, because we are only able to measure one or a few ionization states of a given element (Viegas 1995; Vladilo et al. 2001). The contribution of each of these three sources can theoretically be disentangled through specific abundance ratios and is best constrained through comprehensive sets of elemental abundances within a DLA. There is a degeneracy between these effects that is particularly high for the routinely detected elements (Fe, Si, Cr, Ni, Al, sometimes $\mathrm{Zn}$ ). The specific abundance ratios which should allow to break the degeneracy are: for (i) the ratios involving two elements released in the interstellar medium on different timescales, in particular the $\alpha$ over iron-peak element ratios; for (ii) the ratios of two elements with the same nucleosynthesis origin, but with different dust depletion levels, like [ $\mathrm{Zn} / \mathrm{Fe}]$; and for (iii) the ratios of two elements preferentially with the same nucleosynthesis origin, but with one element being more sensitive to ionization than the other, like $[\mathrm{O} / \mathrm{Si}]$.

Low redshift $\left(z_{\mathrm{abs}}<1\right)$ deep imaging reveals that the DLA galaxies have a variety of morphological types (e.g. Le Brun et al. 1997; Nestor et al. 2002; Rao et al. 2003; Chen \& Lanzetta 2003). If the high redshift DLA galaxy population also samples galaxies with different star formation histories, different stages of chemical evolution, and different interstellar medium (ISM) conditions, we could expect to see the impact of these differences in the chemical abundances, and highlight them through the study of variations in the specific abundance ratios described above. A range of at least $0.3 \mathrm{dex}$ is, for example, observed in the $\alpha$ over iron-peak element ratios, when comparing the abundance measurements of stars in the Milky Way with those of the Small and Large Magellanic Clouds (SMC, LMC) and dwarf spheroidal galaxies and when comparing the abundance measurements of stars within a given galaxy, due to different star formation histories (Venn 1999; Shetrone et al. 2003; Tolstoy et al. 2003). Similarly there is a greater than 0.5 dex range in the gas-phase abundances of different lines of sight crossing the Milky Way and Magellanic Clouds due to different physical conditions, e.g. various dust-to-gas ratios, volume densities and ionization states within the ISM (Savage \& Sembach 1996; Welty et al. 1999, 2001).

Even though DLA galaxies have H I column densities spanning an order of magnitude and metallicities spread over two orders of magnitude, previous studies show that DLAs have similar abundance ratios suggesting that these protogalaxies have common enrichment histories (Prochaska \& Wolfe 2002). In the same way, the abundance patterns of DLA "clouds" for a given system also show uniformity suggesting that the gas of these protogalaxies has a similar enrichment history and uniform differential dust depletion (Prochaska \& Wolfe 1996; Lopez et al. 2002; Prochaska 2003). If confirmed, these results have important implications for the ISM of high redshift galaxies and the enrichment of gas in the early Universe.

In this paper, we further investigate the issue of chemical variations in the abundance ratios of both DLA galaxies and DLA gas along the velocity profiles. We focus on our sample of eleven DLAs with comprehensive sets of elemental abundances. This sample comprises the four DLAs studied in Paper I and the seven new DLAs presented here (Sects. 2 and 3). We discuss the global abundance patterns of DLAs in Sect. 4, and in Sects. 5 and 6 we study the abundance patterns of clouds within the DLA galaxies, which we regard as individual entities. First, we consider all the clouds of all the DLAs from our sample together (Sect. 5), and second we focus on the cloud-to-cloud chemical analysis within a single DLA (Sect. 6). In Sect. 7 we summarize our results. Finally, we present an astrophysical determination of the oscillator strength of the Ni II $\lambda 1317$ transition in Appendix A. In two future papers, Paper III and Paper IV of this series, we will further analyze this sample of DLAs. In Paper III, we will study their star formation history, age, and star formation rate through a detailed comparison with chemical evolution models, and in Paper IV we plan to analyze in detail their ionization state through comparisons of the observed ionic ratios against photoionization models. Two DLAs of our sample are likely to have among the highest ionization levels of all previously studied DLA galaxies.

\section{Observations and data reduction}

The selected quasars Q0450-13, Q0841+129, Q1157+014, Q1210+17, Q2230+02, and Q2348-1444 with seven intervening DLAs in the redshift range $z_{\mathrm{abs}}=1.8-2.5$ are relatively bright with $V=16.5-18.5$. Their spectra have already been reported in the literature, and four of them have been studied by Prochaska \& Wolfe (1999) and Prochaska et al. (2001) using high resolution, high quality spectra obtained with the HIRES echelle spectrograph on the Keck I 10 m telescope at Mauna Kea, Hawaii. More details on these DLA systems and the 
Table 1. Journal of observations.

\begin{tabular}{|c|c|c|c|c|c|c|c|}
\hline Quasar & $\begin{array}{c}V \\
{[\mathrm{mag}]}\end{array}$ & $z_{\mathrm{em}}$ & $z_{\mathrm{abs}}$ & Observing date & UVES Setting & $\begin{array}{l}\text { Wavelength coverage } \\
{[\mathrm{nm}]}\end{array}$ & $\begin{array}{c}\text { Exposure time } \\
{[\mathrm{s}]}\end{array}$ \\
\hline \multirow[t]{2}{*}{ Q0450-13 } & 16.5 & 2.30 & 2.067 & November 2002 & dic1 (B346+R580) & $306-387 / 478-681$ & 6000 \\
\hline & & & & November 2002 & $\operatorname{dic} 2(\mathrm{~B} 346+\mathrm{R} 860)$ & $306-387 / 671-1000$ & 9000 \\
\hline Q0841+129 & 18.5 & 2.50 & $2.375 / 2.476$ & December 2002 & $\operatorname{dic} 2(\mathrm{~B} 390+\mathrm{R} 860)$ & $330-452 / 671-1000$ & 10800 \\
\hline \multirow[t]{3}{*}{ Q1157+014 ${ }^{\dagger}$} & 17.0 & 1.99 & 1.944 & April 2000 & dic1 (B346+R550) & $310-387 / 448-651$ & 7200 \\
\hline & & & & June 2001/January 2002 & dic1 (B380+R580) & $319-441 / 478-681$ & 5400 \\
\hline & & & & June 2001 & $\operatorname{dic} 2(B 380+R 750)$ & $319-441 / 558-939$ & 7200 \\
\hline Q1210+17 & 17.4 & 2.54 & 1.892 & February 2003 & $\operatorname{dic} 2(\mathrm{~B} 346+\mathrm{R} 860)$ & $325-381 / 671-1000$ & 30500 \\
\hline Q2230+02 & 18.0 & 2.15 & 1.864 & October 2003 & $\operatorname{dic} 2(B 346+R 860)$ & $310-387 / 671-1000$ & 13500 \\
\hline Q2348-1444 & 16.9 & 2.94 & 2.279 & November 2002 & $\operatorname{dic} 2(\mathrm{~B} 390+\mathrm{R} 800)$ & $344-452 / 609-990$ & 10350 \\
\hline
\end{tabular}

${ }^{\dagger}$ Based on UVES observations made with the European Southern Observatory VLT/Kueyen telescope obtained from the ESO/ST-ECF Science Archive Facility (programme ID No. 65.O-0063, 67.A-0078, and 68.A-0461).

existing data can be found in Sect. 3. This sample of six QSOs completes our first sample of QSOs, - Q0100+13, Q1331+17, Q2231-00, and Q2343+12 -, with the same characteristics in redshift and magnitude as studied in Paper I. The combination of these two sets of observations provides an impressive sample of eleven damped Ly $\alpha$ systems with comprehensive elemental abundances.

We used the unique capability of the Ultraviolet-Visual Echelle Spectrograph UVES (D'Odorico et al. 2000) on the VLT $8.2 \mathrm{~m}$ Kueyen ESO telescope at Cerro Paranal, Chile, to obtain high resolution, high signal-to-noise ratio spectra of our QSOs in the total optical spectral range, or to complete the existing HIRES/Keck spectra in the blue $\lambda=3060-4500 \AA$ and in the far-red $\lambda=6700-10000 \AA$. The observations were performed in service mode in period 70 from October 2002 to April 2003 (programme ID No. 70.B-0258) under good seeing conditions (between $0.5^{\prime \prime}$ and $1^{\prime \prime}$ ). For one object, Q1157+014, we used spectra from the ESO UVES/VLT archive (programme ID No. 65.O-063, 67.A-0078, and 68.A-0461). For each science exposure, slit widths of $1^{\prime \prime}$ in the blue and of $0.9^{\prime \prime}$ in red were used with a CCD binning of $2 \times 2$, resulting in a resolution $F W H M \simeq 6.9 \mathrm{~km} \mathrm{~s}^{-1}$ and $6.4 \mathrm{~km} \mathrm{~s}^{-1}$ on average, respectively. Relevant details on the observations are collected in Table 1. The total exposure times of each QSO were split in multiple exposures of 3600 or $4500 \mathrm{~s}$.

The spectra were reduced using the ESO data reduction package MIDAS and the UVES pipeline in an interactive mode available as a MIDAS context. A detailed description of the pipeline can be found in Ballester et al. (2000). To optimize the results, we made a systematic check of each step of the pipeline reduction. Once reduced, the wavelengths of the resulting one-dimensional spectra were converted to a vacuumheliocentric scale. For QSOs with multiple exposures, the individual spectra were co-added using their signal-to-noise ratio as weights. The spectra were normalized by smoothly connecting regions free from absorption features with a spline function. In the Ly $\alpha$ forest, the continuum was fitted by using small regions deemed to be free from absorptions and by interpolating between these regions with a spline. An average signal-to-noise ratio per pixel of $\sim 25,45$, and 40 was achieved in the final spectra at $\lambda \sim 3700,7000$, and $9000 \AA$, respectively.

\section{Data analysis and ionic column densities}

By combining our UVES/VLT spectra with the existing HIRES/Keck spectra of Q0841+129, Q1210+17, Q2230+02, and Q2348-1444, we covered the total spectral range from 3060 to $10000 \AA$ for the six selected QSOs. This gave us access to several metal-line transitions of 22 elements for each of their intervening DLAs. In this section we present the derived ionic column density measurements.

The ionic column densities were obtained using the Voigt profile fitting technique. This technique consists of fitting theoretical Voigt profiles to the observed DLA absorption profiles. These profiles are described well as a complex of components, each defined by a redshift $z$, a Doppler parameter $b$, a column density $N$ and the corresponding errors. The fits were performed using an $\chi^{2}$ minimization routine FITLYMAN in MIDAS (Fontana \& Ballester 1995). We assumed that metal species with similar ionization potentials can be fitted using identical component fitting parameters, i.e. the same $b$ (which means that macroturbulent motions dominate over thermal broadening) and the same $z$ in the same component, and allowing for variations from metal species to metal species in $N$ only. We distinguish three categories of metal species with similar ionization potentials: the low-ion transitions (i.e. the neutral and singly ionized species), the intermediate-ion transitions (e.g. Fe III, Al III), and the high-ion transitions (e.g. C IV, Si IV). By using relatively strong (but not saturated) lines to fix the component fitting parameters (the $b$ and $z$ values for each component), we then obtained excellent fitting results even for weak metal lines and for metal lines in the Ly $\alpha$ forest, where the probability of blending is high, by allowing only the column density to vary. We had a sufficient number of relatively strong metal lines to accurately constrain the fitting parameters in the seven DLAs studied that exhibited multicomponent velocity structures.

In Tables 2-8 we present the results of the component per component ionic column density measurements for the fitting model solutions of the low- and intermediate-ion transitions for the seven DLA systems analyzed. The reported errors are the $1 \sigma$ errors on the fits computed by FITLYMAN. These errors do not take the uncertainties on the continuum level determination into account, unless it is mentioned in Sects. 3.1-3.7. For the 
saturated components, the column densities are listed as lower limits. The values reported as upper limits are either cases with significant line blendings by $\mathrm{H}$ I lines in the Ly $\alpha$ forest or telluric lines or cases of non-detection corresponding to $4 \sigma$ limits. By adopting a conservative $4 \sigma$ upper limit based on the statistical error, we account - in part - the continuum error. In Figs. 2-14 (even numbers), we show the best fitting solutions of all the low- and intermediate-ion transitions detected in the seven DLAs studied. In these velocity plots, $v=0$ corresponds to an arbitrary component, and all the identified components are marked by small vertical bars. The thin solid line represents the best fit. The telluric lines have been identified thanks to the spectrum of a hot, fast rotating star taken on the same night as the science exposures.

The neutral hydrogen column densities were measured from the fit of the Ly $\alpha$ damping line profile. The $b$-value was fixed at $20 \mathrm{~km} \mathrm{~s}^{-1}$, and the redshift $z$ was left as a free parameter or fixed at the redshift of the strongest component of the metalline profile depending on the DLA system (see the comments in the following sub-sections). When other lines of higher members of the Lyman series were accessible in our spectra, they were used in parallel to the $\operatorname{Ly} \alpha$ line to derive the H I column density. In Figs. 1-13 (odd numbers), we show the best H I fitting solutions in the seven DLAs studied. The small vertical bar corresponds to the redshift used in the best-fitting solution, and the thin solid line represents the best fit.

Throughout the analysis we have adopted the list of atomic data, laboratory wavelengths and oscillator strengths, compiled by J.X. Prochaska and collaborators (version 0.5) and presented on the web site "the HIRES Damped Ly $\alpha$ Abundance Database" 1 . The most recent measurements of $\lambda_{\text {rest }}$ and $f$-values of the metal-line transitions that impact the abundances of DLAs and their references are reported there. In Appendix A we present an updated value of the Ni II $\lambda 1317$ oscillator strength derived from different $\mathrm{Ni}$ II transitions detected in the DLAs studied. We adopt the solar meteoritic abundances from Grevesse \& Sauval (1998).

\section{1. $Q 0450-13, z_{a b s}=2.067$}

This quasar was discovered by C. Hazard and was first investigated by Jaunsen et al. (1995). The presence of the DLA system at $z_{\text {abs }}=2.067$ on its line of sight was communicated to us by J.X. Prochaska (private communication). This is the first detailed analysis of its chemical composition. The high quality UVES/VLT spectra cover the total spectral range from 3060 to $10000 \AA$. These data permit analysis of some 39 metal-line transitions and help to obtain accurate column density measurements of Fe II, Si II, Al II, S II, N I, P II, Mg II, C II* , Al III, Fe III, and N II. In addition, we were able to put very reliable upper limits to the column densities of Ni II, Cr II, and Ar I and to provide a lower limit to the column density of O I.

The low-ion absorption line profiles of this DLA are characterized by 13 components spread over $230 \mathrm{~km} \mathrm{~s}^{-1}$ in velocity space (see Fig. 2). Their redshifts, $b$-values, and column densities are presented in Table 2. Only the components 4-9 are

\footnotetext{
${ }^{1}$ http://kingpin.ucsd.edu/ hiresdla/
}

detected in weak metal-line transitions, they contain approximately $80 \%$ of the total column density obtained by summing the contribution of the 13 components. The accessible Ni II lines at $\lambda_{\text {rest }}=1741,1751 \AA$, Cr II lines at $\lambda_{\text {rest }}=2056,2062$, $2066 \AA$, and Ar I lines at $\lambda_{\text {rest }}=1048,1066 \AA$ are all so weak that we consider their derived column densities as upper limits. In the context of upper limits, the column density of $\mathrm{P}^{+}$is a borderline case. However, the P II $\lambda 1152$ line has a slightly higher optical depth than the Ni II and Cr II lines, we thus assumed it is a detection. In contrast, the OI line at $\lambda_{\text {rest }}=1302 \AA$ is saturated in the components 4-7, so we only got a lower limit to its total column density. The $\mathrm{Mg}^{+}$column density was obtained from the unsaturated $\operatorname{Mg}$ II $\lambda$ 1239, 1240 lines. Because these Mg II transitions are located in the red wing of the DLA Ly $\alpha$ damping profile, it is difficult to obtain an accurate column density measurement of $\mathrm{Mg}^{+}$. To estimate the column density, we first normalized the spectra within the damped Ly $\alpha$ profile near the Mg II lines according to the fit of the Ly $\alpha$ damping wing profile. We applied the same procedure to the N I triplet at $\lambda_{\text {rest }} \simeq 1200 \AA$, which is located in the blue wing of the DLA Ly $\alpha$ damping profile. The $1 \sigma$ errors on the measured $N\left(\mathrm{Mg}^{+}\right)$ and $N\left(\mathrm{~N}^{0}\right)$ column densities were estimated by varying the continuum level by $5 \%$. No Zn II line was detected in this DLA system. We provide a $4 \sigma$ upper limit to the $\mathrm{Zn}^{+}$column density of $\log N(\mathrm{Zn}$ II $)<11.60$.

The intermediate-ion absorption line profiles show a different velocity structure than the low-ion line profiles (see Fig. 2). Their fitting solution is presented in the second part of Table 2. However, in velocity space the positions of the 6 components characterizing the intermediate-ion line profiles correspond very closely to the positions of components $1,3,4,6$, and 8 of low-ion lines. When considering the column density ratios of different ionization species of the same element, we find the following very interesting results: $\log N\left(\mathrm{Al}^{++}\right) / N\left(\mathrm{Al}^{+}\right)=-0.77 \pm 0.08, \log N\left(\mathrm{Fe}^{++}\right) / N\left(\mathrm{Fe}^{+}\right)$ $=+0.04 \pm 0.04$ larger than -1 , and $\log N\left(\mathrm{~N}^{+}\right) / N\left(\mathrm{~N}^{0}\right)=+0.88 \pm$ 0.04 larger than -0.2 . Those ratios give a qualitative "firstlook" analysis of the ionization state in a DLA. According to the photoionization diagnostics described by Prochaska et al. (2002a), we have clear evidence in this DLA of a very high ionization level. This will be further discussed in Paper IV of this series. In Sect. 6 we present additional indications toward the presence of strong ionization in this system.

Finally, Fig. 1 shows the fitting solution of two Lyman lines of this DLA, Ly $\alpha$ and $\operatorname{Ly} \beta$. The fits were obtained by fixing the $b$-value at $20 \mathrm{~km} \mathrm{~s}^{-1}$ and the redshift at $z=2.06666$, i.e. at the redshift of one of the stronger metal-line components (the component 6 at $v=-14 \mathrm{~km} \mathrm{~s}^{-1}$ ). The derived H I column density is $\log N(\mathrm{HI})=20.53 \pm 0.08$. This is a relatively high value, especially in light of the evidence mentioned above for the high ionization level in this DLA (Vladilo et al. 2001).

\section{2. $Q 0841+129, z_{a b s}=2.375$}

The two DLA systems at $z_{\mathrm{abs}}=2.375$ and at $z_{\mathrm{abs}}=2.476$ toward the quasar Q0841+129 were identified by C. Hazard and were first studied at low resolution by Pettini et al. (1997). 
More recently, high resolution spectra obtained with HIRES/Keck and UVES/VLT were analyzed by Prochaska \& Wolfe (1999), Prochaska et al. (2001), Centurión et al. (2003), Ledoux et al. (2003), and Vladilo et al. (2003).

In the case of the first DLA at $z_{\mathrm{abs}}=2.375$, we confirm the Prochaska \& Wolfe (1999) and Prochaska et al. (2001) column density measurements of Fe II, Si II, Ni II, Cr II, and Zn II. However, our column density measurements of Fe II, Zn II, S II, and Ar I differ by almost 0.1 dex from those of Centurión et al. (2003) and Vladilo et al. (2003). This discrepancy is likely to be the result of the lower signal-to-noise ratio in their UVES/VLT spectra and an underestimation of their uncertainties. In the cases of $\mathrm{Fe}^{+}, \mathrm{Zn}^{+}$, and $\mathrm{Ar}^{0}$, we used at least two different metalline transitions to derive the column densities; e.g. for $N\left(\mathrm{Fe}^{+}\right)$ 9 transitions are considered (see Fig. 4). We, nevertheless, confirm the Centurión et al. (2003) $N\left(\mathrm{~N}^{0}\right)$ measurement, obtained in our analysis using both the N I triplet at $\lambda_{\text {rest }} \sim 1134$ and $\sim 1200 \AA$, except the N I $\lambda 1134.165$ line which is blended with $\mathrm{H}$ I lines in the Ly $\alpha$ forest.

From a total of 46 metal-line transitions detected and analyzed (see Fig. 4), we obtained the column density measurements of Mg II, Mn II, P II, C II*, Al III, and Fe III, in addition to the ions discussed above. We also derived lower limits to the column densities of $\mathrm{O}$ I and $\mathrm{Al}$ II and upper limits to $N(\mathrm{C}$ I $)$ and $N(\mathrm{~S}$ III).

The low-ion absorption line profiles of this DLA are characterized by a simple velocity structure composed of 6 components spread over $80 \mathrm{~km} \mathrm{~s}^{-1}$. Their properties are described in Table 3. Only the components 3 and 4 are detected in the weaker metal-line transitions. They contain, however, $~ 95 \%$ of the total column density of the fully integrated profile. Due to the high H I column density of this DLA system and the proximity of the second DLA at $z_{\mathrm{abs}}=2.476$ on the same QSO line of sight, the N I triplet at $\lambda_{\text {rest }} \sim 1200 \AA$ is located in the blue wing of the DLA Ly $\alpha$ damping line profile, the $\operatorname{Mg}$ II $\lambda 1239,1240$ lines in the red wing of the DLA Ly $\alpha$ line, and the S II $\lambda 1259$ line, the only unblended S II line available, in the red wing of the $\operatorname{Ly} \alpha$ damping line profile of the second DLA. Consequently, we renormalized the damped Ly $\alpha$ profiles of both DLAs according to their best values, in order to derive accurate column densities of $\mathrm{N}^{0}, \mathrm{Mg}^{+}$, and $\mathrm{S}^{+}$. The $1 \sigma$ errors on the measured column densities are estimated by varying the continuum level by 5\%. The S II $\lambda 1259$ line shows a slight asymmetry in its profile compared to other low-ion metal lines, which is not perfectly modeled in our solution. The Mg I $\lambda 2026$ line in Fig. 4 is plotted to illustrate that there is no contamination of $\mathrm{Zn}$ II $\lambda 2026$ by $\mathrm{Mg}^{0}$.

The intermediate-ion line profiles of this DLA are composed of only two components with very similar characteristics to the main components 3 and 4 of the low-ion lines (see the second part of Table 3 ). The derived $\mathrm{S}^{++}$column density measurement is a borderline case between a detection and an upper limit due to possible blends with $\mathrm{HI}$ lines in the Ly $\alpha$ forest. To be conservative, we assumed it is an upper limit. Following the Prochaska et al. (2002a) photoionization diagnostics, we used the column density ratios of different ionization species of the same element measured in this DLA to infer the ionization state. Specifically the ratios $-\log N\left(\mathrm{Al}^{++}\right) / N\left(\mathrm{Al}^{+}\right)<-1.22, \log N\left(\mathrm{Fe}^{++}\right) / N\left(\mathrm{Fe}^{+}\right)=-0.94 \pm$ 0.04 , and $\log N\left(\mathrm{~S}^{++}\right) / N\left(\mathrm{~S}^{+}\right)<-0.53$ - indicate that this system has a low ionization level, with an ionization fraction, defined as the ratio of $\mathrm{H}^{+}$over $\left(\mathrm{H}^{0}+\mathrm{H}^{+}\right)$, lower than $10 \%$.

The determination of the H I column densities from the Ly $\alpha$ damping lines of the two DLAs toward Q0841+129 was particularly difficult in this case because of the proximity of the two systems (separated by $9040 \mathrm{~km} \mathrm{~s}^{-1}$ only). The detections of the $\operatorname{Ly} \beta$ line in the DLA at $z_{\mathrm{abs}}=2.375$ and the $\operatorname{Ly} \beta$ and Ly $\gamma$ lines in the DLA at $z_{\mathrm{abs}}=2.476$ in our UVES/VLT spectra provide an indirect check on the fits. In Figs. 3 and 5, we show the derived results for the two DLAs. In both systems, we fitted all the lines of the Lyman series simultaneously, and we fixed the $b$-value at $20 \mathrm{~km} \mathrm{~s}^{-1}$ and the redshift at $z=2.37452$ and $z=2.47621$, respectively, i.e. at the redshift of the strongest metal-line component (the component 4 and 2, respectively). The derived H I column densities, $\log N(\mathrm{HI})=20.99 \pm 0.08$ for the first DLA and $\log N(\mathrm{HI})=20.78 \pm 0.08$ for the second DLA, are in a very good agreement with the values from Centurión et al. (2003) and Vladilo et al. (2003).

\section{3. $Q 0841+129, Z_{a b s}=2.476$}

As mentioned in Sect. 3.2, this DLA system toward Q0841+129 has already been studied by several authors at high resolution. Using our UVES/VLT spectra combined with the existing HIRES/Keck spectra, we obtained a high signalto-noise ratio and a total optical wavelength coverage, which allowed us to analyze 45 different metal-line transitions (see Fig. 6). We obtained the column density measurements of Fe II, Si II, N I, S II, O I, Ar I, Mg II, P II, Mn II, Ni II, Cr II, Zn II, and Al III. We also derived lower limits to the column densities of C II and Al II from saturated lines, and upper limits to the column densities of C II* ${ }^{*}$ Fe III, N II, and S III. Discrepancies between 0.05 and 0.1 dex are observed between our column density measurements and those obtained by Prochaska \& Wolfe (1999), Prochaska et al. (2001), Centurión et al. (2003), and Vladilo et al. (2003). In the majority of cases, we used a larger number of metal-line transitions to measure the column density of a given ion, which appreciably increases the accuracy of the measurement, especially in those cases where the transitions lie outside the Ly $\alpha$ forest. In addition, in the case of the N I, S II, and Ar I lines, we benefited from a signal-to-noise ratio that is twice as high as the spectra used by Centurión et al. (2003) and Vladilo et al. (2003).

The low-ion absorption line profiles of this DLA are characterized by only 3 components spread over $20 \mathrm{~km} \mathrm{~s}^{-1}$ (see Table 4). The component 1 is only observed in the stronger metal-line transitions and it contributes negligibly to the total column density. In weak metal-line transitions, the component 3 is only marginally detected, like for instance in ArI, P II, Mn II, and Ni II (see Fig. 6). Thus, for several ions we adopted large errors on their component 3 column density measurements. The column density measurement of $\mathrm{O}^{0}$ is a borderline case between a detection and a limit. It was derived from the saturated $\mathrm{O}$ I $\lambda 1039$ line, which gives a constraint on the lower limit to $N\left(\mathrm{O}^{0}\right)$ and the O I $\lambda 950$ line far in the blue, 
exposed to several possible line blendings, which provides a constraint on the upper limit to $N\left(\mathrm{O}^{0}\right)$. By fitting these two lines simultaneously, we assumed that we get a reliable column density measurement of $\mathrm{O}^{0}$. Granted the large uncertainty, we adopted a conservative error estimate. The determination of $N\left(\mathrm{P}^{+}\right)$was also challenging. However, the value derived from the P II $\lambda 963$ line, far in the blue (perhaps blended with H I lines in the Ly $\alpha$ forest), agrees perfectly with the weak P II $\lambda 1152$ line. Therefore, we assumed the measured $N\left(\mathrm{P}^{+}\right)$value is a good detection. Finally, the measurements of the column densities of $\mathrm{Mg}^{+}$and $\mathrm{Zn}^{+}$are borderline cases between detections and upper limits. Indeed, the observed Zn II $\lambda 2026,2062$ and $\operatorname{Mg}$ II $\lambda 1239,1240$ lines are very weak, detected at $3 \sigma$ only. Therefore, we report conservatively large errors for their column density measurements.

In the intermediate-ion line profiles of this DLA, we detected a single component which has the same characteristics as the strongest component of low-ion lines, component 2 (see Table 4). We obtained the column density measurements of four intermediate-ions, $\mathrm{Al}^{++}, \mathrm{Fe}^{++}, \mathrm{N}^{+}$, and $\mathrm{S}^{++}$. To be conservative, we considered three of them, $-N\left(\mathrm{Fe}^{++}\right), N\left(\mathrm{~N}^{+}\right)$, and $N\left(\mathrm{~S}^{++}\right)-$, as upper limits due to possible blends with $\mathrm{H}$ I lines in the Ly $\alpha$ forest (see Fig. 6). These measurements lead to the following column density ratios for different ionization species of the same element: $\log N\left(\mathrm{Al}^{++}\right) / N\left(\mathrm{Al}^{+}\right)<-0.78$, $\log N\left(\mathrm{Fe}^{++}\right) / N\left(\mathrm{Fe}^{+}\right)<-0.95, \log N\left(\mathrm{~N}^{+}\right) / N\left(\mathrm{~N}^{0}\right)<-0.40$, and $\log N\left(\mathrm{~S}^{++}\right) / N\left(\mathrm{~S}^{+}\right)<-0.76$. According to the photoionization diagnostics of Prochaska et al. (2002a), these ratios show the gas is predominantly neutral.

\section{4. $Q 1157+014, Z_{\mathrm{abs}}=1.944$}

This DLA system was first identified by Wolfe \& Briggs (1981) at $21 \mathrm{~cm}$. They obtained an estimation of its $\mathrm{H}^{0}$ column density of $\log N(\mathrm{HI})=21.80 \pm 0.10$. This value is an important reference to check our measurement of $N(\mathrm{HI})$ derived from UVES/VLT high resolution spectra. In Fig. 7 we show the Ly $\alpha$ line of the DLA system. Only the blue wing is plotted, because of the very uncertain normalization of the red Ly $\alpha$ damping wing, given its proximity to the QSO Ly $\alpha$ emission peak $(\lambda \sim 3635 \AA)$. By fixing the $b$-value at $20 \mathrm{~km} \mathrm{~s}^{-1}$ and the redshift at $z=1.94366$, i.e. at the redshift of the strongest metalline component (the component 4 ), we obtained a good fit of the blue Ly $\alpha$ damping wing profile. The measured H I column density, $\log N(\mathrm{HI})=21.60 \pm 0.10$, is consistent with the value obtained by Wolfe \& Briggs (1981). In addition, our measurement is in a very good agreement with the $\mathrm{H}^{0}$ column density, $\log N(\mathrm{H} \mathrm{I})=21.70 \pm 0.10$, also measured from high resolution spectra by Ledoux et al. (2003).

This DLA, with its high H I column density and high metallicity $\left(>10^{21} \mathrm{~cm}^{-2},[\mathrm{Zn} / \mathrm{H}]=-1.27 \pm 0.12\right)$, belongs to the category of "metal-strong" DLA systems defined by Prochaska et al. (2003). These systems show strong metal absorption lines and, as a consequence, allow the ionic column density measurements of a very large number of ions and elements. Indeed, in this DLA system, for instance, 54 metal-line transitions were detected (see Fig. 8). We obtained accurate column density measurements of Fe II, Si II, Mg II, P II, C II*, Mn II, Ni II, Cr II, Zn II, Ti II, Co II, Ge II, Mg I, and Al III. We also derived lower limits to the column densities of $\mathrm{Al}$ II and $\mathrm{N} \mathrm{I}$ and upper limits to the column densities of C I, B II, Cl I, Cu II, Kr I, and As II.

The low-ion absorption line profiles of this DLA are characterized by 7 components spread over $145 \mathrm{~km} \mathrm{~s}^{-1}$. Their redshifts, $b$-values, and column densities are presented in Table 5. The components 1 and 7 are only observed in the stronger metal lines and make a negligible contribution to the total ionic column densities. The problem of contamination of the Zn II $\lambda 2026$ line by the MgI $\lambda 2026$ line was first raised by Prochaska et al. (2001). To control the impact of this blend on the $\mathrm{Zn}^{+}$column density measurement, we needed to know the level of the contribution of $\mathrm{Mg}^{0}$ in the $\mathrm{Zn}$ II line. For this purpose, we used the $\operatorname{Mg} \mathrm{I} \lambda 2852$ line, which gives an accurate estimate of the contamination at $2026 \AA$ (see Fig. 8). One drawback of the high H I column density of this DLA is that the blue Ly $\alpha$ damping line wing overlaps the N I triplet at $\lambda_{\text {rest }} \sim$ $1200 \AA$ A. Therefore, we locally renormalized the spectrum with the fit of the Ly $\alpha$ damping wing profile to derive a column density of $\mathrm{N}^{0}$. An additional difficulty related to the $N(\mathrm{~N} \mathrm{I})$ measurement is that the profile is probably saturated. Indeed, the components 5 and 6 look almost saturated even in the weaker N I transitions, e.g. in the lines at $\lambda_{\text {rest }}=1134.415,1134.980$, and $1200.710 \AA$, and the weakest N I line at $\lambda_{\text {rest }}=1134.165 \AA$ is strongly blended with $\mathrm{HI}$ lines in the Ly $\alpha$ forest to be relevant. We are thus inclined to assume that the derived $\mathrm{N}^{0}$ column density is a lower limit. In contrast, the P II column density measurement is a borderline case between a detection and an upper limit. Indeed, the P II $\lambda 1152$ line shows signs of possible blends with $\mathrm{HI}$ lines in the Ly $\alpha$ forest mainly toward the blue edge. However, its fit agrees perfectly with the weaker P II $\lambda 1532$ line (see Fig. 8). We thus assume that the measured $N\left(\mathrm{P}^{+}\right)$is a detection.

The high H I column density and the relatively high metallicity of this DLA allowed us to obtain column density measurements and reliable upper limits of some very weak metalline transitions. We obtained a very accurate column density measurement of Ti II from the detection and analysis of several Ti II lines at $\lambda_{\text {rest }}=1910.609,1910.938,3067$, and $3073 \AA$. More exceptionally, at $3 \sigma$ we detected the Co II $\lambda 2012$ and Ge II $\lambda 1237$ lines (see Fig. 8). Only two detections of $\mathrm{Co}^{+}$in DLAs have been reported by Ellison et al. (2001) and Rao et al. (2005) and one previous measurement of $\mathrm{Ge}^{+}$by Prochaska et al. (2003). We are concerned that the observed Co and Ge profiles do not show significant optical depth at $v=0$, but we suppose that this is within the statistical error adopted on their column density measurement. We also obtained very reliable $4 \sigma$ upper limits to the column densities of $\mathrm{B}^{+}, \mathrm{Cl}^{0}, \mathrm{Cu}^{+}, \mathrm{Kr}^{0}$, and $\mathrm{As}^{+}$from the undetected but clean, i.e. free from blending and with a high signal-to-noise ratio, B II $\lambda 1362, \mathrm{Cl}$ I $\lambda 1347$, Cu II $\lambda$ 1358, KrI $\lambda$ 1164, and As II $\lambda 1355$ lines. Previously, only Prochaska et al. (2003) have put meaningful upper limits to the column densities of these ions in another metal-strong DLA system.

The intermediate-ion line profiles have the same shape and characteristics as the low-ion line profiles in this DLA (see Fig. 8 and Table 5). We obtained the column density 
measurement of $\mathrm{Al}$ III and an upper limit to $N(\mathrm{Fe}$ III) from the Fe III $\lambda 1122$ line located in the $\operatorname{Ly} \alpha$ forest and probably blended with $\mathrm{HI}$ lines, by using the same fitting parameters as those defined for the low-ion lines. The measured very low column density ratio, $\log N\left(\mathrm{Al}^{++}\right) / N\left(\mathrm{Al}^{+}\right)<-1.82$, clearly shows that the ionization is negligible in this DLA, strongly self-shielded by the high neutral hydrogen column density gas (Prochaska et al. 2002a).

\section{5. $Q 1210+17, Z_{a b s}=1.892$}

This DLA system toward Q1210+17 was discovered by Wolfe et al. (1995) and is a member of the Large Bright QSO Survey. It has already been studied at high resolution by Prochaska et al. (2001). Using our UVES/VLT spectra combined with their HIRES/Keck spectra, we confirm their column density measurements of Fe II, Si II, Ni II, Cr II, and Zn II at 0.05 dex. In addition, we obtained the column density measurements of S II, $\mathrm{N}$ I, Mn II, Ti II, and Al III, lower limits to the column density of $\mathrm{Al}$ II and $\mathrm{Mg}$ II, and an upper limit to the column density of P II from a total of 45 metal-line transitions analyzed (see Fig. 10).

The low-ion absorption line profiles of this DLA are characterized by a simple velocity structure, composed of 7 components spread over $130 \mathrm{~km} \mathrm{~s}^{-1}$. Their properties are described in Table 6. Only the components 3, 4, and 5 were detected in the weak metal-line transitions. They contain nearly all of the column density of the profile ( $\sim 95 \%)$. The derived column density of $\mathrm{N}^{0}$ is a borderline case between a detection and an upper limit due to possible blends with $\mathrm{H}$ I lines in the Ly $\alpha$ forest. All the available $\mathrm{N}$ I lines seem to be blended, although luckily not over the whole profile. In this way, we succeeded in constraining the column densities of components 3,4 , and 5 by fitting them to the various $\mathrm{N}$ I lines. We believe that we obtained a reliable $N\left(\mathrm{~N}^{0}\right)$ but report a conservatively large error estimate. The measurement of the column density of $\mathrm{Mg}^{+}$is also tricky. The saturated Mg II $\lambda 2796,2803$ lines provide a lower limit to $N\left(\mathrm{Mg}^{+}\right)$, while the $\mathrm{Mg}$ II $\lambda 1239,1240$ lines in the Ly $\alpha$ forest give an upper limit to the column density of the component 4 . The upper limit to the column density of $\mathrm{P}^{+}$was obtained from the P II $\lambda$ 1152, 1532 lines. The $\mathrm{Mg}$ I $\lambda 2026$ line in Fig. 10 illustrates that the $\mathrm{Zn}$ II $\lambda 2026$ line is not contaminated by $\mathrm{Mg}^{0}$.

The intermediate-ion lines of this DLA show exactly the same profiles as the low-ion lines (see Fig. 10 and Table 6). We obtained the column density measurement of $\mathrm{Al}^{++}$only. This DLA has a very low column density ratio $\log N\left(\mathrm{Al}^{++}\right) / N\left(\mathrm{Al}^{+}\right)<-1.96$, which clearly indicates that the ionization is very low in this system (Prochaska et al. 2002a).

Figure 9 shows the fitting solution of the Ly $\alpha$ line of this DLA. The fit was obtained by fixing the $b$-value at $20 \mathrm{~km} \mathrm{~s}^{-1}$ and the redshift at $z=1.89177$, i.e. at the redshift of the strongest metal-line component (the component 4 ). The derived $\mathrm{H}$ I column density is $\log N(\mathrm{HI})=20.63 \pm 0.08$, which agrees well with the value obtained by Wolfe et al. (1995) from low resolution spectra. This DLA system exhibits a high metallicity of $[\mathrm{Zn} / \mathrm{H}]=-0.88 \pm 0.10$, and hence almost belongs to the category of metal-strong DLAs (Prochaska et al. 2003). Therefore, we were able to derive a very accurate $\mathrm{Ti}^{+}$column density measurement from the detection of several Ti II lines at $\lambda_{\text {rest }}=3067,3073,3242$, and $3384 \AA$. In addition, we obtained reliable $4 \sigma$ upper limits to the column densities of $\mathrm{Kr}^{0}$ and $\mathrm{As}^{+}$ from the undetected $\operatorname{Kr} I \lambda 1164$ and As II $\lambda 1263$ lines, respectively. The Ge II $\lambda 1237$ line is unfortunately blended, and the B II $\lambda 1362$, Cu II $\lambda$ 1358, O I $\lambda 1355$, and Co II lines are not covered in our high signal-to-noise ratio UVES/VLT spectra, with which we could probably have provided reliable upper limits or detections of these ions in this DLA (see Sect. 3.4).

\section{6. $Q 2230+02, Z_{\mathrm{abs}}=1.864$}

This DLA system is an object from the Large Bright QSO Survey (Wolfe et al. 1995). It has been studied at low resolution by Pettini et al. (1994), and more recently at high resolution by Prochaska \& Wolfe (1999) and Prochaska et al. (2001). Prochaska \& Wolfe (1999) have analyzed this DLA extensively thanks to the detection of 28 metal-line transitions in their HIRES/Keck spectra. We confirm their column density measurements of Fe II, Si II, Ni II, Cr II, and Al III at 0.05 dex. However, our column density measurements of $\mathrm{Zn} \mathrm{II}$ and Ti II differ by more than 0.05 dex, as described below. From a total of 46 metal-line transitions detected and analyzed (see Fig. 12), we obtained, in addition, the column density measurements of S II, N I, P II, Mn II, C II*, and Mg I, and an upper limit to the column density of Fe III.

The low-ion absorption line profiles of this DLA are characterized by a very complex velocity structure extended over $400 \mathrm{~km} \mathrm{~s}^{-1}$ in velocity space and composed of 21 components presented in Table 7 . Three important clumps at -41 , +17 , and $+74 \mathrm{~km} \mathrm{~s}^{-1}$ composed of mainly 4 components, the components $9,12,13$, and 14 , dominate the profile. They contain about $75 \%$ of the total column density obtained by summing the contribution of the 21 components. This complex velocity structure of low-ion lines made the determination of the $\mathrm{N}^{0}$ column density very difficult. Indeed, due to the large velocity spread of the profiles, the three lines of the $\mathrm{N}_{\mathrm{I}}$ triplets at $\lambda_{\text {rest }} \sim 1134$ and at $\sim 1200 \AA$ are blended together, but luckily not too heavily contaminated by $\mathrm{H}$ I lines in the Ly $\alpha$ forest (see Fig. 12). By careful work on the 6 N I lines available in our UVES/VLT spectra and by using the fitting parameters constrained with unblended and uncontaminated low-ion lines, we finally derived an accurate $\mathrm{N}^{0}$ column density. The $\mathrm{Zn}^{+}$column density measurement also needed some attention, due to a possible blend of the Zn II $\lambda 2026$ line with the $\mathrm{Mg}$ I $\lambda 2026$ line. The $\mathrm{Mg}$ I $\lambda 2852$ line allowed us to very accurately determine the contamination of the $\mathrm{Zn} \mathrm{II} \mathrm{column}$ density by $\mathrm{Mg}^{0}$ by obtaining a fit of the $\mathrm{Mg}$ I $\lambda 2026$ line. This contamination is lower than $0.1 \mathrm{dex}$, but it explains the discrepancy between Prochaska \& Wolfe (1999) $\mathrm{Zn}^{+}$column density measurement, $\log N(\mathrm{Zn}$ II $)=12.80 \pm 0.03$, and our weaker value, $\log N(\mathrm{Zn}$ II $)=12.72 \pm 0.05$.

In the saturated $\mathrm{O}$ I $\lambda 1302$ and $\mathrm{Al}$ II $\lambda 1670$ lines, we measured the column densities of the unsaturated components only (see Table 7). The column density of the component 9 of $\mathrm{C}^{*}{ }^{*}$ is referred to as an upper limit because the blue edge of the $\mathrm{CII}^{*} \lambda 1335$ line is blended with the $\mathrm{CII} \lambda 1334$ line. 
The $\operatorname{Mg}$ II $\lambda 1239,1240$ lines were undetected in this DLA, so we provide only a $4 \sigma$ upper limit to the column density of $\mathrm{Mg}^{+}$. Despite the low signal-to-noise ratio per pixel of 8 in the wavelength regions covering the S II and P II lines, we obtained accurate $\mathrm{S}^{+}$and $\mathrm{P}^{+}$column densities. Finally, we derived a very accurate column density measurement of $\mathrm{Ti}^{+}$, thanks to the access to several Ti II lines at $\lambda_{\text {rest }}=1910.609,1910.938$, 3073, 3242, and $3384 \AA$. Our $N\left(\mathrm{Ti}^{+}\right)$differs from the one of Prochaska \& Wolfe (1999) obtained from the two Ti II lines at $\lambda_{\text {rest }} \sim 1910 \AA$, which have a 2-3 times weaker oscillator strength than the Ti II $\lambda 3242,3384$ lines used in our measurement.

Only 11 components were detected in the intermediateion line profiles of this DLA. They show very similar characteristics as the components 5 to 15 of the low-ion lines (see the second part of Table 7 and Fig. 12). We obtained a column density measurement of $\mathrm{Al}^{++}$and an upper limit to $N\left(\mathrm{Fe}^{++}\right)$from the Fe III $\lambda 1122$ line probably blended with $\mathrm{H}$ I lines in the Ly $\alpha$ forest. The measured low column density ratios, $\log N\left(\mathrm{Al}^{++}\right) / N\left(\mathrm{Al}^{+}\right)<-0.43$ and $\log N\left(\mathrm{Fe}^{++}\right) / N\left(\mathrm{Fe}^{+}\right)<$ -0.61 , clearly show that this DLA system has a low ionization level (Prochaska et al. 2002a).

While fitting the Ly $\alpha$ damping line profile at $z=1.86375$ (redshift of one of the stronger metal-line components, the component 12), we found it necessary to include the contribution of a second absorber shifted by about $550 \mathrm{~km} \mathrm{~s}^{-1}$ bluewards the DLA system (see Fig. 11). The redshift of this second absorber, $z=1.85851$, was accurately determined from several associated metal lines. Pettini et al. (1994) already discovered the presence of these two closeby absorption line systems in their low resolution spectra by observing that the metal lines consist of two main components separated by $\sim 550 \mathrm{~km} \mathrm{~s}^{-1}$. The derived $\mathrm{H}$ I column densities are $\log N(\mathrm{HI})=20.83 \pm 0.05$ for the DLA and $\log N(\mathrm{HI})=20.00 \pm 0.10$ for the second $a b-$ sorber. They agree well with the values obtained by Pettini et al. (1994).

\section{7. $Q 2348-1444, z_{a b s}=2.279$}

This DLA system was first discussed by Pettini et al. (1994) and first studied at high resolution by Pettini et al. (1995). Subsequently it was carefully analyzed by Prochaska \& Wolfe (1999) and Prochaska et al. (2002b) using HIRES/Keck spectra. We confirm their column density measurements of Fe II, Si II, S II, Al II, and C II* at 0.05 dex, and their lower limit to the column density of OI derived from the saturated O I $\lambda 1302$ line. From a total of 36 metal-line transitions detected and analyzed (see Fig. 14), we obtained the column density measurements of N I, Mn II, Cr II, Al III, and Fe III, in addition, and upper limits to the column densities of $\mathrm{Mg}$ II, Ni II, Zn II, and S III.

The low-ion absorption line profiles of this DLA are characterized by a very simple velocity structure composed of 4 components spread over $40 \mathrm{~km} \mathrm{~s}^{-1}$. Their properties are described in Table 8 . Component 4 was only detected in the stronger metal-line transitions and its contribution to the total column density is negligible. Many metal lines observed in this DLA are very weak, and their column density measurements are borderline cases between detections and upper limits. This is the case for the Mg II, Mn II, Ni II, Cr II, and $\mathrm{Zn}$ II lines. Only the Mn II and Cr II lines were detected at $3 \sigma$; hence, by adopting large errors we obtained reliable $\mathrm{Mn}^{+}$ and $\mathrm{Cr}^{+}$column densities. For the other lines detected at less than $3 \sigma$, we provided valuable upper limits. While the very weak $\mathrm{Mg}$ II $\lambda 1239,1240$ lines gave an upper limit to $N\left(\mathrm{Mg}^{+}\right)$, the saturated $\mathrm{Mg}$ II $\lambda 2796,2803$ lines led to a lower limit to $N\left(\mathrm{Mg}^{+}\right)$(see Table 8). We consider the derived $\mathrm{C} \mathrm{II}^{*}$ column density in agreement with the value obtained by Prochaska $\&$ Wolfe (1999) as an upper limit due to possible blends of the $\mathrm{C} \mathrm{II}^{*} \lambda 1335$ line with H I lines in the Ly $\alpha$ forest. Similarly we prefer to assume that the measured $\mathrm{Al}$ II column density is an upper limit due to a possible blend of the Al II $\lambda 1670$ line with a metal line (see Fig. 14). Thanks to our access to several $\mathrm{N}$ I lines, we could derive a value for the first time and not only an upper limit to the $\mathrm{N}^{0}$ column density (Pettini et al. 1995; Prochaska et al. 2002b). However, before fitting the N I lines, we first had to locally renormalize the spectrum around the N I triplet at $\lambda_{\text {rest }} \sim 1200 \AA$ with the fit of the blue DLA Ly $\alpha$ damping wing profile.

The intermediate-ion lines of this DLA show exactly the same profiles as the low-ion lines (see Fig. 14 and Table 8). We obtained the column density measurements of $\mathrm{Al}^{++}$and $\mathrm{Fe}^{++}$ from the Fe III $\lambda 1122$ line located in the Ly $\alpha$ forest. The Fe III line might be blended with $\mathrm{H}$ I lines, so we adopted a large error on $N\left(\mathrm{Fe}^{++}\right)$. We also derived an upper limit to the column density of S III. These measurements lead to the following column density ratios of different ionization species of the same element: $\log N\left(\mathrm{Al}^{++}\right) / N\left(\mathrm{Al}^{+}\right)>-0.66, \log N\left(\mathrm{Fe}^{++}\right) / N\left(\mathrm{Fe}^{+}\right)=$ $-0.45 \pm 0.14$, and $\log N\left(\mathrm{~S}^{++}\right) / N\left(\mathrm{~S}^{+}\right)<+0.04$. According to the photoionization diagnostics of Prochaska et al. (2002a), these ratios show that the DLA system is likely to be partially ionized. The ionization fraction is, however, lower than $50 \%$ and the expected ionization corrections on the measured ionic column densities are on the order of only $0.1 \mathrm{dex}$. The most important corrections to the observed gas-phase abundances are that the intrinsic $[\mathrm{Si} / \mathrm{Fe}]$ ratio is slightly lower and the intrinsic $[\mathrm{N} / \mathrm{Si}, \mathrm{S}]$ ratios are slightly higher.

Figure 13 shows the fitting solution of the Ly $\alpha$ line of this DLA system. The fit was obtained by fixing the $b$-value at $20 \mathrm{~km} \mathrm{~s}^{-1}$ and the redshift at $z=2.27939$, i.e. at the redshift of the strongest metal-line component (component 3 ). The derived $\mathrm{HI}$ column density is $\log N(\mathrm{HI})=20.59 \pm 0.08$, which agrees well with the value obtained by Pettini et al. (1994) from low resolution spectra.

\section{Global gas-phase abundance patterns}

In the next three sections we will use the results obtained for the seven DLAs studied here (see Sect. 3) in combination with the results derived in Paper I for four DLAs. These eleven DLAs constitute the first sample of high redshift objects for which we have the following at our disposal: accurate column density measurements of individual interstellar medium "clouds" within the DLAs detected along the velocity profiles; 

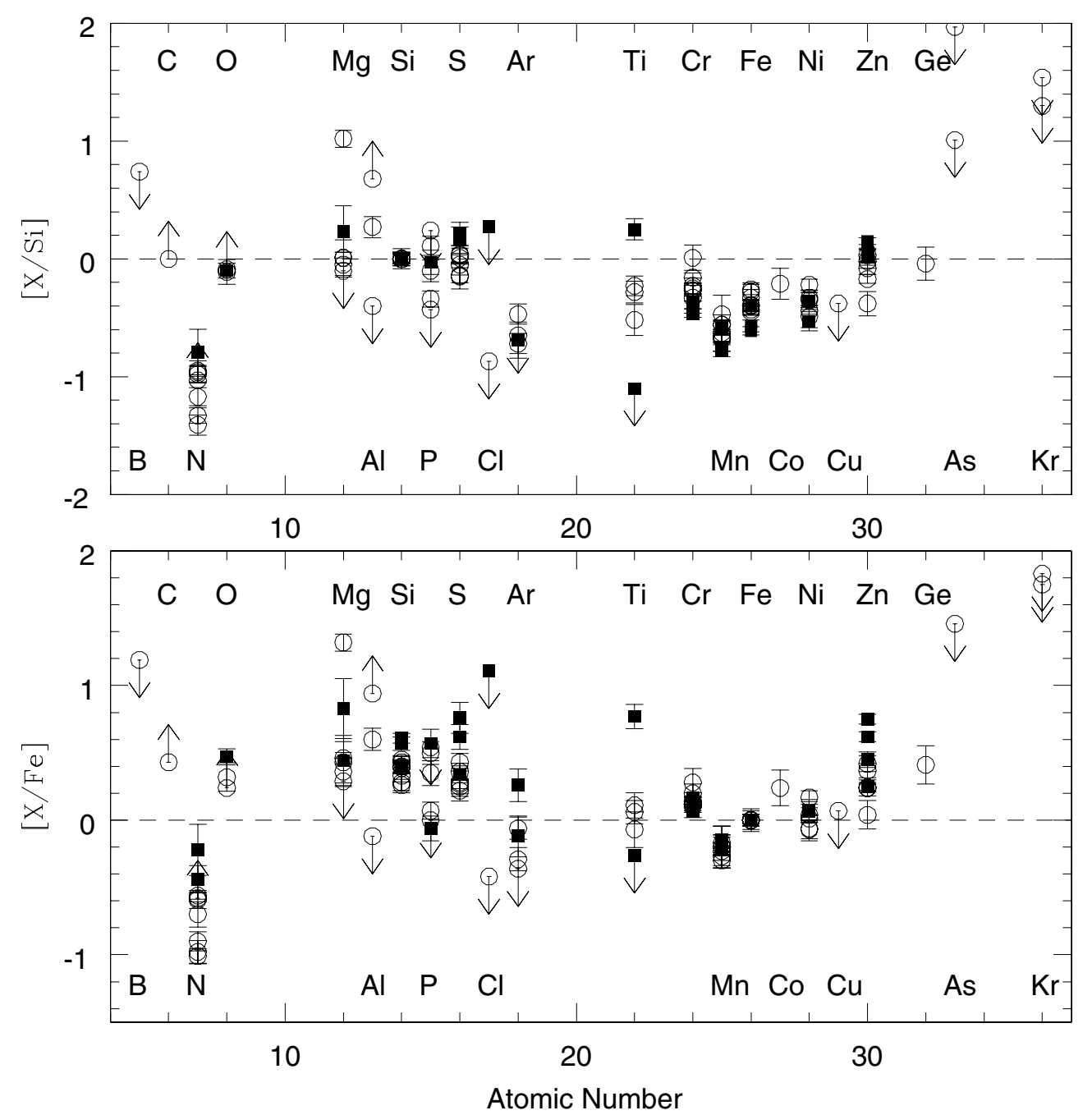

Fig. 15. Nucleosynthetic abundance patterns $[\mathrm{X} / \mathrm{Si}]$ (upper panel) and $[\mathrm{X} / \mathrm{Fe}]$ (lower panel) for our sample of 11 DLAs. We consider the entire set of detected elements X covering a large range of atomic numbers from 5 to 36 . The plotted abundance ratios are the raw abundance ratio measurements, i.e. the directly observed gas-phase abundance ratios, free from any correction (dust or ionization). Our sample of data is composed of the 7 DLAs studied in this paper (open circles) and of 4 DLAs analyzed in Paper I (filled squares). In Table 9 we present some statistical results relative to the observed abundance pattern trends.

the column density measurements of up to 30 ions; and the abundance measurements of up to 22 elements.

The absolute abundances, $[\mathrm{X} / \mathrm{H}]$, are obtained by summing the contributions of all the components detected in the element $\mathrm{X}$ profile and reported in Tables 2-8. In what follows we will discuss the abundance ratios, $[\mathrm{X} / \mathrm{Y}]$. These are computed by considering only the column densities of components detected in both the element $\mathrm{X}$ and $\mathrm{Y}$ profiles. This is equivalent to comparing $\mathrm{X}$ and $\mathrm{Y}$ over the exact same velocity intervals. In this way we prevent an overestimation of abundances derived from strong metal-line profiles relative to the abundances derived from weaker metal-line profiles in which only the stronger components are usually detected. In the case of very weak metal lines, like the Ti II lines for instance, one can indeed underestimate the $[\mathrm{X} / \mathrm{Fe}]$ ratios by up to $0.3-0.4$ dex by considering the total $\mathrm{Fe}$ abundance generally derived by summing a much larger number of components than for the Ti abundance. This effect is particularly important in DLAs with complex metal-line profiles extended over a large velocity range, in which a large difference is observed between the number of components detected in strong and weak metal lines. In our sample this is mainly the case of the DLAs toward Q0450-13 and toward Q2230+02. The same approach was already used in Paper I, where the DLAs toward Q2231-00 and toward Q2343+12 were subject to such uncertainties.

Figure 15 shows the nucleosynthetic abundance patterns as a function of the atomic number of the seven DLAs studied in this paper (open circles) and the four DLAs studied in Paper I (filled squares). We present both the $[\mathrm{X} / \mathrm{Si}]$ and $[\mathrm{X} / \mathrm{Fe}]$ abundance ratios relative to the solar values for the entire set of 22 detected elements $\mathrm{X}$ covering a range of atomic numbers from 5 to 36. This contrasts with the majority of DLAs for which only a handful of elements is usually detected (e.g. Lu et al. 1996; Prochaska \& Wolfe 1999; Prochaska et al. 2001). We consider here the raw abundance ratios free from any correction (dust or ionization). In this way we can try to identify whether the SFH, dust depletion, or ionization has a perceptible impact on some abundance ratios, and we can study the 
dispersion in the abundance patterns of the DLA galaxy population. In Table 9 we present some statistical results on the observed abundance pattern trends. We computed the logarithmic weighted mean of the $[\mathrm{X} / \mathrm{Si}]$ and $[\mathrm{X} / \mathrm{Fe}]$ abundance ratios using their $1 \sigma$ errors as weights, the logarithmic rms dispersion in $[\mathrm{X} / \mathrm{Si}]$ and $[\mathrm{X} / \mathrm{Fe}]$ ratios, and the reduced $\chi^{2}$ of $[\mathrm{X} / \mathrm{Si}]$ and $[\mathrm{X} / \mathrm{Fe}]$ relative to their weighted mean. The numbers in parentheses are given for information only. They correspond to the abundance ratios $[\mathrm{X} / \mathrm{Si}]$ and $[\mathrm{X} / \mathrm{Fe}]$ computed by considering all of the gas in the Si II and Fe II profiles, in comparison to the adopted method for the computation of the $[\mathrm{X} / \mathrm{Si}]$ and $[\mathrm{X} / \mathrm{Fe}]$ ratios (see the beginning of this section). To be conservative we do not take the limits in these calculations into account. We do note, however, that the limits would only lead to an increased dispersion in abundance ratios in which the detections already show a dispersion, e.g. P and Ti (see Fig. 15).

We underline that, although the abundance ratio measurements presented here are the raw ones (i.e. the directly observed gas-phase abundances), they show a remarkable uniformity $^{2}$. Indeed, in the 11 DLA systems studied with redshifts between 1.7 and 2.5 , H I column densities covering one order of magnitude from $2 \times 10^{20}$ to $4 \times 10^{21} \mathrm{~cm}^{-2}$, and metallicities from $1 / 55$ to $1 / 5$ solar, the abundance patterns show relatively low rms dispersions, reaching only up to 2-3 times higher values than the statistical errors ${ }^{3}$ for the majority of elements. This suggests that the effects of nucleosynthesis enrichment, dust depletion, and ionization are negligible and that the abundance ratios of the integrated profiles for the DLA galaxy population are very uniform, as pointed out by Molaro (2005). In turn, this implies the respective star formation histories have conspired to yield one set of relative abundances. The time interval sampled by the DLA galaxies studied is of $1.8 \mathrm{Gyr}$ for the adopted $H_{0}=65 \mathrm{~km} \mathrm{~s}^{-1} \mathrm{Mpc}^{-1}, \Omega_{\mathrm{M}}=0.3$, and $\Omega_{\Lambda}=0.7$ cosmology.

We now discuss element per element the results derived for the $[\mathrm{X} / \mathrm{Si}]$ and $[\mathrm{X} / \mathrm{Fe}]$ ratios, along with their implications:

$N$ : The $[\mathrm{N} / \mathrm{Si}]$ and $[\mathrm{N} / \mathrm{Fe}]$ ratios both show a large dispersion, larger than 0.2 dex, with [N/Si] values ranging from -0.8 to -1.5 . We expect that the large dispersion is due to different star formation histories (SFH) and ages of DLA galaxies, as discussed by a number of authors (see Pettini et al. 2002; Prochaska et al. 2002b; Centurión et al. 2003; Chiappini et al. 2003). We do not wish to review the details here. While variations in the star formation histories are most likely the principal source of $[\mathrm{N} / \mathrm{Si}]$ and $[\mathrm{N} / \mathrm{Fe}]$ dispersions, ionization effects may also be important. Indeed, $\mathrm{N}^{0}$ has a larger cross-section to photons with $h v>2$ Ryd than $\mathrm{H}^{0}$ (Sofia \& Jenkins 1998). Both in the DLA toward Q0450-13 and the DLA toward Q2343+12 with strong evidence for high ionization levels, we observe that a significant fraction of $\mathrm{N}$ is in the form of $\mathrm{N}^{+}$and not $\mathrm{N}^{0}$ only. As a consequence $\mathrm{N}^{0} /\left(\mathrm{Si}^{+}, \mathrm{Fe}^{+}\right)$will underestimate $\mathrm{N} /(\mathrm{Si}, \mathrm{Fe})$ (Prochaska et al. 2002b).

\footnotetext{
2 This uniformity is even stronger when the abundance ratios $[\mathrm{X} / \mathrm{Si}]$ and $[\mathrm{X} / \mathrm{Fe}]$ are computed by considering all of the gas in the Si II and Fe II profiles (values in parenthesis in Table 9).

3 The statistical errors are defined as the average of the $1 \sigma$ errors on measurements.
}

Table 9. Abundance patterns of the integrated profiles for our sample of 11 DLAs.

\begin{tabular}{|c|c|c|c|c|}
\hline Element & \# DLAs & Mean $^{a}$ & $\mathrm{rms}^{b}$ & $\chi_{v}^{2 c}$ \\
\hline \multicolumn{5}{|l|}{$[\mathrm{X} / \mathrm{Si}]$} \\
\hline$N \ldots$ & 7 & $-1.089(-1.126)$ & $0.225(0.195)$ & 7.65(7.772) \\
\hline $\operatorname{Mg} \ldots$ & 5 & $0.238(0.197)$ & $0.447(0.405)$ & $38.37(30.345)$ \\
\hline $\mathrm{P} \ldots \ldots$ & 4 & $-0.127(-0.173)$ & $0.193(0.176)$ & $6.44(4.872)$ \\
\hline S & 8 & $0.001(-0.032)$ & $0.129(0.121)$ & $2.06(1.785)$ \\
\hline & 3 & $-0.608(-0.662)$ & $0.138(0.200)$ & $2.31(2.666)$ \\
\hline $\mathrm{Ti} \ldots$. & 4 & $-0.147(-0.295)$ & $0.327(0.226)$ & $10.28(5.114)$ \\
\hline $\mathrm{Cr} \ldots$ & 9 & $-0.314(-0.359)$ & $0.148(0.186)$ & $6.01(9.229)$ \\
\hline Mn ... & 9 & $-0.656(-0.695)$ & $0.102(0.140)$ & $3.57(5.688)$ \\
\hline $\mathrm{Fe} \ldots$ & 10 & $-0.436(-0.436)$ & $0.115(0.115)$ & $6.27(6.266)$ \\
\hline $\mathrm{Ni} \ldots$. & 7 & $-0.404(-0.417)$ & $0.109(0.087)$ & $4.07(1.945)$ \\
\hline & 8 & $0.007(-0.042)$ & $0.174(0.177)$ & $9.73(10.444)$ \\
\hline \multicolumn{5}{|l|}{$[\mathrm{X} / \mathrm{Fe}]$} \\
\hline $\mathrm{N}$. & 8 & $-0.678(-0.706)$ & $0.278(0.244)$ & $12.96(13.428)$ \\
\hline $\mathrm{Mg} \ldots$ & 6 & $0.685(0.645)$ & $0.376(0.353)$ & $28.22(23.584)$ \\
\hline $\mathrm{Si} \ldots$. & 10 & $0.430(0.430)$ & $0.114(0.114)$ & $6.63(6.630)$ \\
\hline & 5 & $0.241(0.193)$ & $0.277(0.272)$ & $9.62(9.265)$ \\
\hline S. & 9 & $0.331(0.303)$ & $0.195(0.191)$ & $4.22(3.699)$ \\
\hline $\mathrm{Ar}$ & 4 & $-0.092(-0.140)$ & $0.234(0.270)$ & $4.64(5.150)$ \\
\hline $\mathrm{Ti}$. & 4 & $0.261(0.076)$ & $0.379(0.233)$ & $15.59(6.765)$ \\
\hline $\mathrm{Cr} \ldots$ & 10 & $0.131(0.092)$ & $0.060(0.107)$ & $0.66(3.582)$ \\
\hline Mn ... & 9 & $-0.227(-0.271)$ & $0.053(0.077)$ & $1.69(2.515)$ \\
\hline $\mathrm{Ni} \ldots$. & 7 & $0.022(0.007)$ & $0.085(0.068)$ & $2.42(1.174)$ \\
\hline $\mathrm{Zn} \ldots$ & 9 & $0.396(0.329)$ & $0.217(0.199)$ & $26.51(23.241)$ \\
\hline
\end{tabular}

Note. The numbers in parentheses correspond to the abundance ratios $[\mathrm{X} / \mathrm{Si}]$ and $[\mathrm{X} / \mathrm{Fe}]$ computed by considering all of the gas in the Si II and Fe II profiles in comparison to the adopted method (see Sect. 4).

${ }^{a}$ Logarithmic weighted mean of $[\mathrm{X} / \mathrm{Si}]$ and $[\mathrm{X} / \mathrm{Fe}]$ using their $1 \sigma$ errors as weights.

${ }^{b}$ Logarithmic rms dispersion in $[\mathrm{X} / \mathrm{Si}]$ and $[\mathrm{X} / \mathrm{Fe}]$.

${ }^{c}$ Reduced $\chi^{2}$ of $[\mathrm{X} / \mathrm{Si}]$ and $[\mathrm{X} / \mathrm{Fe}]$ relative to their weighted mean.

$M g$ : Aside from the measurement of Srianand et al. (2000), the six $\mathrm{Mg}$ abundance measurements from our sample are the only $\mathrm{Mg}$ abundances derived in DLAs to date. The large dispersions observed in the $[\mathrm{Mg} / \mathrm{Si}]$ and $[\mathrm{Mg} / \mathrm{Fe}]$ ratios of +0.45 and +0.38 dex, respectively, are due to the upper open circle which corresponds to the DLA toward Q0450-13. This DLA shows several clues for a high ionization fraction (see Sect. 3.1). Hence, the measured abundance of $\mathrm{Mg}^{+}$is easily overestimated by several tenths of dex (see Fig. 5 in DessaugesZavadsky et al. 2002), which leads to the high $[\mathrm{Mg} / \mathrm{Si}]$ and $[\mathrm{Mg} / \mathrm{Fe}]$ ratios observed in this DLA. $\mathrm{Mg}$ is an $\alpha$-element and it should at least roughly trace $\mathrm{Si}$ as observed in Galactic metalpoor stars (François et al. 2004), although $\mathrm{Si}$ is probably produced in non-negligible amounts by type Ia supernovae ( $\mathrm{SNe}$ ) in addition to type II SNe. This is confirmed by our data, when excluding the DLA toward Q0450-13.

Si: The low $[\mathrm{Si} / \mathrm{Fe}]$ dispersion $\left(0.11 \mathrm{dex}, \chi_{v}^{2}=6.6\right)$ indicates that the $[\mathrm{Si} / \mathrm{Fe}]$ ratio remains nearly constant irrespective of the DLA galaxy and its dust depletion level. The mean $[\mathrm{Si} / \mathrm{Fe}]$ value of +0.43 dex then suggests on average a high $\alpha$-element enhancement relative to iron-peak elements in all DLA systems, which is an enrichment by massive stars. Indeed, 
the $\alpha$-elements are produced in less than $2 \times 10^{7}$ yrs by type II $\mathrm{SNe}$ resulting from massive stars, while the iron-peak elements are mainly produced by type Ia SNe on longer timescales between $3 \times 10^{7}$ and $10^{9}$ yrs (Matteucci \& Recchi 2001).

$P$ : $\quad$ P cannot be measured in Galactic halo stars; thus the recent entry of $\mathrm{P}$ in the set of elements observed in DLAs offers a unique astronomical site where it can be measured at metallicities significantly lower than solar. The $[\mathrm{P} / \mathrm{Si}]$ and $[\mathrm{P} / \mathrm{Fe}]$ ratios show a large dispersion. The $[\mathrm{P} / \mathrm{Si}]$ ratio is particularly interesting for observing the odd-even effect as it corresponds to an underabundance of odd-Z elements relative to even- $Z$ elements of the same nucleosynthetic origin. The mean $[\mathrm{P} / \mathrm{Si}]$ value of -0.13 shows evidence of a mild odd-even effect, lower than the expectations on the basis of yields by Woosley \& Weaver (1995) and Limongi et al. (2000). However, some DLAs show a strong odd-even effect, as the DLA at $z_{\mathrm{abs}}=2.375$ toward $\mathrm{Q} 0841+129$ with $[\mathrm{P} / \mathrm{Si}]=-0.34 \pm 0.09$. This leads to a high $[\mathrm{P} / \mathrm{Si}]$ dispersion due to various strengths of the odd-even effect from galaxy to galaxy. We emphasize that $\mathrm{P}$ is a non-refractory element and the variations are not very likely to be related to differential depletion.

$S: \quad$ The $[\mathrm{S} / \mathrm{Si}]$ ratio shows a relatively low dispersion and a mean solar value. This implies that $S$ traces $S i$ very closely as in Galactic halo stars. Hence, the refractory $\alpha$-element Si appears to be almost insensitive to dust depletion effects at the level they are observed in the DLAs studied, since it traces the volatile $\alpha$-element $\mathrm{S}$ whatever the dust depletion. The $[\mathrm{S} / \mathrm{Fe}] \mathrm{ra}-$ tio shows a large rms dispersion of 0.19 dex, larger than the one for the $[\mathrm{Si} / \mathrm{Fe}]$ ratio. As a consequence, it might appear that the $[\mathrm{S} / \mathrm{Fe}]$ ratio is more subject to variations due to dust depletion or nucleosynthesis enrichment than the $[\mathrm{Si} / \mathrm{Fe}]$ ratio, but only in the order of the weak variations allowed by the $[\mathrm{S} / \mathrm{Si}]$ ratios. The low reduced $\chi^{2}$ of the $[\mathrm{S} / \mathrm{Fe}]$ ratio values of 4.2 confirms that the $[\mathrm{S} / \mathrm{Fe}]$ intrinsic variations are in fact low and that $[\mathrm{S} / \mathrm{Fe}]$ remains almost constant as $[\mathrm{Si} / \mathrm{Fe}]$, whatever the DLA galaxy and its dust depletion level might be. The mean $[\mathrm{S} / \mathrm{Fe}]$ value of +0.33 dex suggests, on average, an $\alpha$-element enhancement in all DLA systems as the $[\mathrm{Si} / \mathrm{Fe}]$ ratio.

$\mathrm{Cl}$ : We obtained a measurement and an upper limit to the column density of $\mathrm{Cl}$ I. They both yield upper limits to the $\mathrm{Cl}$ abundance, since the dominant state of $\mathrm{Cl}$ in DLAs is $\mathrm{Cl}^{+}$and not $\mathrm{Cl}^{0}$. Indeed, most of $\mathrm{Cl} \mathrm{I}$ is probably ionized, given that the ionization potential of $\mathrm{Cl}^{0}$ is lower than $1 \mathrm{Ryd}$. Unfortunately, no general abundance pattern trend can be discussed for this element (see Paper I for a description of its importance).

Ar: $\mathrm{Ar}$ is a typical product of type II SNe. It is presumed to track other $\alpha$-elements, although there is little empirical evidence. No Ar abundance measurement exists in Galactic stars, and thus Ar measurements in DLAs are of high priority in the search to better understand the behavior of this element. The measured $[\mathrm{Ar} / \mathrm{Si}]$ ratios have a low dispersion of $0.14 \mathrm{dex}$ with a very low reduced $\chi^{2}$. Interestingly, all the obtained Ar measurements show significant underabundances relative to $\mathrm{Si}$, the mean $[\mathrm{Ar} / \mathrm{Si}$ ] value being -0.61 . No theoretical yields of $\mathrm{Ar}$ may explain such a large underabundance of Ar relative to $\mathrm{Si}$, and the $[\mathrm{Ar} / \mathrm{Si}]$ ratio is expected to be only weakly dependent on the SFH undergone by DLAs. Dust depletion effects are also not likely to explain these underabundances, since $\mathrm{Ar}$ is non-refractory. The only explanation can be found in the ionization effects. Indeed, ArI is very sensitive to ionization, in particular because its photoionization cross-section is one order of magnitude larger than for H I (Sofia \& Jenkins 1998). Hence, Lyman continuum photons with energies $h v>2$ Ryd are more efficient in ionizing ArI than $\mathrm{HI}$, if they are able to leak through the H I layer (Vladilo et al. 2003). We wish to emphasize, however, that although all of our DLAs show a significant Ar underabundance, other ionization diagnostics imply the gas is predominantly neutral for the majority of our DLAs. Therefore, the $[\mathrm{Ar} / \mathrm{Si}$ ] ratio alone cannot characterize the ionization fraction of a DLA.

$\mathrm{Ti}$ : $\mathrm{Ti}$ is generally accepted as an $\alpha$-element, because it exhibits abundance patterns similar to other $\alpha$-elements in Galactic stars (Edvardsson et al. 1995; François et al. 2004). It is a refractory element and has a high dust depletion level, even higher than Fe in Galactic ISM clouds (Savage \& Sembach 1996). The dust depletion effects thus explain the large dispersion in the $[\mathrm{Ti} / \mathrm{Si}]$ ratios of $0.33 \mathrm{dex}$, and the mean value of -0.15 is suggestive of some presence of dust in the majority of DLAs studied. The large dispersion in the $[\mathrm{Ti} / \mathrm{Fe}]$ ratios of 0.38 dex is due both to dust depletion and nucleosynthesis enrichment effects. Positive departures of [Ti/Fe] from the solar value is evidence of an $\alpha$-enhancement, and negative $[\mathrm{Ti} / \mathrm{Fe}]$ ratios provide evidence of dust depletion (see Dessauges-Zavadsky et al. 2002).

$\mathrm{Cr}, \mathrm{Ni}, \mathrm{Fe}: \mathrm{Cr}, \mathrm{Ni}$, and $\mathrm{Fe}$ are three iron-peak elements with refractory properties. They trace each other in Galactic stars, but in gas phase the $[\mathrm{Cr} / \mathrm{Fe}]$ and $[\mathrm{Ni} / \mathrm{Fe}]$ ratios may show small differences from the solar value, due to differential dust depletion. All the DLAs studied show an enhanced $[\mathrm{Cr} / \mathrm{Fe}]$ ratio, the mean value being +0.13 dex in agreement with Prochaska \& Wolfe (2002) findings. This is suggestive of dust depletion, since Galactic ISM lines of sight do exhibit a mild $\mathrm{Cr}$ overabundance relative to $\mathrm{Fe}$. However, it is difficult to explain why every DLA shows enhanced $[\mathrm{Cr} / \mathrm{Fe}]$ and why there is no trend with $[\mathrm{Zn} / \mathrm{Fe}]$ (for the $\mathrm{Zn}$ discussion see below). The mean $[\mathrm{Ni} / \mathrm{Fe}]$ value is solar, as it is observed in the Galactic ISM. These two abundance ratios show no dispersion, and they are uniform from one DLA to another with an rms of 0.06 and 0.08 dex, respectively. This uniformity can be explained by the fact that $\mathrm{Cr}, \mathrm{Ni}$, and $\mathrm{Fe}$ have very similar dust depletion patterns in the Galactic ISM (Savage \& Sembach 1996), and the DLAs do not have enough high dust depletion variations from one system to another to produce $[\mathrm{Cr} / \mathrm{Fe}]$ and $[\mathrm{Ni} / \mathrm{Fe}]$ variations.

$\mathrm{Mn}$ : $\mathrm{Mn}$ is an iron-peak element, but it behaves differently from other iron-peak elements. The $[\mathrm{Mn} / \mathrm{Fe}]$ ratios in Galactic stars are undersolar and show a decrease with metallicity. They illustrate a nice example of the odd-even effect for iron-nuclei. All the Mn abundance measurements obtained in the DLAs studied are also underabundant relative to $\mathrm{Fe}$ with a $[\mathrm{Mn} / \mathrm{Fe}]$ mean value of -0.23 . In addition, these measurements are very uniform with an rms of 0.05 dex. This uniformity implies two important consequences. Firstly, the DLA [Mn/Fe] ratios reach a plateau at -0.23 dex with a small scatter for a metallicity of DLA systems between $[\mathrm{Zn} / \mathrm{H}]=-2$ and $-0.7 \mathrm{dex}$. Secondly, the $[\mathrm{Mn} / \mathrm{Fe}]$ ratios are similar irrespective of the dust depletion level of DLAs, as we have already argued in 
Dessauges-Zavadsky et al. (2002), since Mn has a very similar dust depletion level to that of Fe. In a future paper, we will consider if this result contradicts the conclusions of McWilliam et al. (2003) based on stellar abundances that Mn is a secondary element.

Co: We obtained the third Co abundance measurement in the DLA system toward Q1157+014. The first ones were obtained by Ellison et al. (2001) and Rao et al. (2005). Co is an ironpeak element. Its dust depletion in the Galactic ISM is not well known; Co has probably a refractory nature similar to the one of Fe. In Galactic stars, [Co/Fe] shows a large scatter around the solar value. No general DLA abundance pattern trend can be discussed for this element yet. The three $[\mathrm{Co} / \mathrm{Fe}]$ measurements are: $[\mathrm{Co} / \mathrm{Fe}]=+0.24 \pm 0.13$ (our value), $[\mathrm{Co} / \mathrm{Fe}]=+0.31 \pm$ 0.05 (Ellison et al. 2001), and $[\mathrm{Co} / \mathrm{Fe}]=+0.05 \pm 0.12(\mathrm{Rao}$ et al. 2005).

$\mathrm{Zn}$ : $\mathrm{Zn}$ is an extremely important element in DLA abundance studies. It is frequently considered as an iron-peak element because it traces the other iron-peak elements in Galactic stars, but $\mathrm{Zn}$ is probably produced through different nucleosynthetic processes (e.g. Matteucci et al. 1993). Zn has a unique trump, which is that it is not readily incorporated into dust grains and thus is only very mildly refractory (Savage \& Sembach 1996). As a consequence, the $[\mathrm{Zn} / \mathrm{Fe}]$ ratio is widely used as a tracer of the dust depletion level in a DLA, with higher $[\mathrm{Zn} / \mathrm{Fe}]$ values implying higher depletion levels. Our measurements show a high dispersion of $0.22 \mathrm{dex}$ in the $[\mathrm{Zn} / \mathrm{Fe}]$ ratios and a reduced $\chi^{2}$ of 26 . The $[\mathrm{Zn} / \mathrm{Fe}]$ values vary from 0 to +0.8 , which indicates that the DLAs studied sample a relatively large range of dust depletion levels. The [ $\mathrm{Zn} / \mathrm{Si}$ ] ratio is relatively free from dust depletion effects, since, as discussed above, the refractory $\alpha$-element $\mathrm{Si}$ traces the volatile element $\mathrm{S}$ well whatever the dust depletion level might be in the DLAs studied. Hence, the $[\mathrm{Zn} / \mathrm{Si}]$ ratio is a good tracer of only nucleosynthetic enrichment. The $[\mathrm{Zn} / \mathrm{Si}]$ dispersion of $0.17 \mathrm{dex}$ with a $\chi_{v}^{2}$ of 10 is relatively large, and its most straightforward explanation can be found in different SFHs from one DLA to another. However, this dispersion, as well as the mean solar $[\mathrm{Zn} / \mathrm{Si}]$ value, contradict the results obtained for $[\mathrm{Si} / \mathrm{Fe}]$ and $[\mathrm{S} / \mathrm{Fe}]$. Indeed, as discussed above, the $[\mathrm{Si} / \mathrm{Fe}]$ and $[\mathrm{S} / \mathrm{Fe}]$ ratios seem to be uniform and enhanced relative to solar values. How can we reconcile the solar $[\mathrm{Zn} / \mathrm{Si}]$ abundance pattern with the $\alpha$-enhancement observed in the $[\mathrm{Si} / \mathrm{Fe}]$ and $[\mathrm{S} / \mathrm{Fe}]$ ratios that cannot entirely be accounted for by dust depletion effects? The only solution is to assume that the $[\mathrm{Zn} / \mathrm{Fe}]$ ratio can be larger than 0 , independently from dust depletion effects. Recent measurements of Galactic metal-poor stars indeed suggest that $\mathrm{Zn}$ is overabundant relative to $\mathrm{Fe}$ in the range of 0 to +0.2 dex for metallicities between $[\mathrm{Fe} / \mathrm{H}]=-2$ and -1 (Prochaska \& McWilliam 2000; Mishenina et al. 2002; Nissen et al. 2004; Chen et al. 2004; François et al. 2004).

Ge: $\mathrm{Ge}$ is the element with the highest atomic number $(\mathrm{Z}=32)$ for which we obtained an abundance measurement. Prochaska et al. (2003) were the first to draw attention to the prospect of measuring Ge in DLAs. It is an element of the iron-peak, but the s-process in massive stars may also contribute to its production. It thus has a secondary origin, since it can only be produced when Fe is already present in stars.
Cowan et al. (2005) have very recently shown that Ge abundances in Galactic metal-poor stars track the Fe abundances very well, but at a depressed level, $\langle[\mathrm{Ge} / \mathrm{Fe}]\rangle=-0.79 \pm 0.04$. Hence, an explosive process on iron-peak nuclei, rather than neutron capture, appears to be the dominant synthesis mechanism for $\mathrm{Ge}$ at low metallicities. At higher metallicities, the s-process production takes place, and it would be expected that the $\mathrm{Ge}$ abundances would increase with the $\mathrm{Fe}$ abundances. In DLAs, the Ge abundances do not seem to follow the same trend relative to the $\mathrm{Fe}$ abundances. Indeed, the two measured $[\mathrm{Ge} / \mathrm{Fe}]$ abundance ratios are oversolar, $[\mathrm{Ge} / \mathrm{Fe}]=+0.41 \pm 0.15$ (our measurement) and $[\mathrm{Ge} / \mathrm{Fe}]=+0.77$ (Prochaska et al. 2003).

$B, A s, K r$ : We just obtained upper limits to the abundances of these elements. They will be discussed in a future paper.

\section{Gas-phase abundance patterns of individual "clouds"}

From the Voigt profile fitting of metal-lines, we obtained very accurate component-to-component column density measurements. These components presumably correspond to interstellar medium clouds in the DLA galaxy on the QSO line of sight. We consider here these clouds as individual entities and study their abundance pattern trends.

It is important, however, to define the concept of a cloud more strictly relative to the components defined in the Voigt profile fits. Indeed, to be able to consider the column density of a component as a physical property of an individual ISM cloud on a QSO line of sight, the component has to be independent, i.e. unblended with its neighbors. We assumed two components are independent if they satisfy the following criterion: the difference of position, $\Delta v$, in velocity space between two components has to be larger than the sum of their half line widths (i.e. their half $b$-values) within $5 \sigma$ of their respective errors. The results of this criterion agree well with an eye-ball determination of independent components from optical depth considerations. The components which do not satisfy this criterion are encompassed in Tables 2-8 and together they form an independent cloud. Their column densities have to be summed and this sum corresponds to the column density of the cloud. The same criterion has been applied to the DLAs studied in Paper I. In our sample of 11 DLA systems, we find a total of 84 clouds.

In the first part of Table 10, we present the dispersion properties for different abundance ratios $-[\mathrm{Si} / \mathrm{Fe}],[\mathrm{S} / \mathrm{Fe}],[\mathrm{Si} / \mathrm{Zn}]$, [S/Zn], [S/Si], [N/Si], [Mn/Fe], [Cr/Fe], and [Zn/Fe] - of all the clouds observed in our sample of 11 DLA galaxies. We give the logarithmic weighted mean computed using the $1 \sigma$ errors as weights in Col. (3), the logarithmic rms dispersion in Col. (4), and the reduced $\chi^{2}$ relative to the weighted mean in Col. (5). We notice that, in general, the mean abundance ratio values of clouds are very similar to those of integrated profiles of DLAs, but the measured rms dispersions and reduced $\chi^{2}$ are 2-3 times larger for individual clouds than the values obtained for the DLAs (see Table 9). This is partly due to the larger statistical errors in the cloud abundance ratio measurements.

However, we do observe statistically significant dispersions of about $5 \sigma$ in the $[\mathrm{Zn} / \mathrm{Fe}]$ ratio and larger than $3 \sigma$ in the 
Table 10. Abundance patterns and correlations between $[\mathrm{X} / \mathrm{Y}]$ ratios and $[\mathrm{Zn} / \mathrm{Fe}]$ for all clouds observed in our sample of 11 DLAs.

\begin{tabular}{l|crrr|rrrrr}
\hline \hline$[\mathrm{X} / \mathrm{Y}]$ & \# clouds $^{1}$ & \multicolumn{1}{c}{ Mean } & \multicolumn{1}{c}{ rms } & \multicolumn{1}{c}{$\chi_{v}^{2}$} & \# clouds $^{2}$ & $\tau^{(a)}$ & $P(\tau)^{(b)}$ & $a^{(c)}$ & $b^{(d)}$ \\
\hline$[\mathrm{Si} / \mathrm{Fe}]$ & 79 & 0.390 & 0.183 & 18.10 & 22 & 0.686 & 0.000 & $+0.241 \pm 0.023$ & $+0.534 \pm 0.044$ \\
{$[\mathrm{~S} / \mathrm{Fe}]$} & 34 & 0.376 & 0.251 & 9.85 & 18 & 0.757 & 0.000 & $+0.060 \pm 0.038$ & $+0.961 \pm 0.083$ \\
{$[\mathrm{Si} / \mathrm{Zn}]$} & 22 & -0.033 & 0.173 & 11.60 & 22 & -0.712 & 0.000 & $+0.246 \pm 0.025$ & $-0.495 \pm 0.041$ \\
{$[\mathrm{~S} / \mathrm{Zn}]$} & 18 & 0.032 & 0.101 & 1.62 & 18 & -0.343 & 0.047 & $+0.058 \pm 0.032$ & $-0.059 \pm 0.071$ \\
{$[\mathrm{~S} / \mathrm{Si}]$} & 32 & 0.047 & 0.213 & 5.03 & 16 & 0.561 & 0.002 & $-0.152 \pm 0.037$ & $+0.416 \pm 0.079$ \\
{$[\mathrm{~N} / \mathrm{Si}]$} & 36 & -1.005 & 0.318 & 17.04 & 12 & 0.412 & 0.062 & no correlation \\
{$[\mathrm{Mn} / \mathrm{Fe}]$} & 29 & -0.221 & 0.108 & 5.37 & 22 & 0.264 & 0.086 & no correlation \\
{$[\mathrm{Cr} / \mathrm{Fe}]$} & 28 & 0.130 & 0.105 & 2.89 & 24 & 0.097 & 0.508 & no correlation \\
{$[\mathrm{Zn} / \mathrm{Fe}]$} & 25 & 0.427 & 0.263 & 30.01 & & & &
\end{tabular}

Note. For the definition of a "cloud", see Sect. 5.

${ }^{1}$ Total number of clouds in our sample of 11 DLAs with a measurement of [X/Y]. The corresponding logarithmic weighted mean, logarithmic rms dispersion, and reduced $\chi^{2}$ are given in columns (3), (4), and (5), respectively.

${ }^{2}$ Number of clouds in our sample of 11 DLAs with both a measurement of [X/Y] and [Zn/Fe]. Data plotted in Fig. 16.

${ }^{(a)}$ Kendall correlation factor of $[\mathrm{X} / \mathrm{Y}]$ versus $[\mathrm{Zn} / \mathrm{Fe}]$. A positive value of $\tau$ corresponds to a correlation and a negative value to an anti-correlation.

(b) Probability under the null hypothesis of zero correlation from the Kendall test. A value $<5 \%$ indicates a significant correlation.

(c), (d) Zero point and slope, respectively, and their $1 \sigma$ uncertainties, of the linear least-square regression $[\mathrm{X} / \mathrm{Y}]=a+b \times[\mathrm{Zn} / \mathrm{Fe}]$, computed by taking into account the errors on both $[\mathrm{X} / \mathrm{Y}]$ and $[\mathrm{Zn} / \mathrm{Fe}]$ data points.

$[\alpha / \mathrm{Fe}, \mathrm{Zn}]$ ratios, except for $[\mathrm{S} / \mathrm{Zn}]$ which has a particularly low dispersion ${ }^{4}$. High dispersions are expected according to the observations made in the Milky Way and the Local Group galaxies, if these clouds probe different physical conditions and come from galaxies with various SFHs. Indeed, the observed gas-phase abundance ratios along a Galactic line of sight may vary by more than $0.5 \mathrm{dex}$ as the sightline penetrates clouds arising in various phases of the ISM (e.g. Savage \& Sembach 1996). Similarly, the LMC and SMC also exhibit large variations in their gas-phase abundance ratios (Welty et al. 1999, 2001). This is due to the fact that sightlines through the ISM probe gas with a range of physical conditions, e.g. various dustto-gas ratios, volume densities, and ionization states. In addition, a range of at least $0.3 \mathrm{dex}$ in the $\alpha / \mathrm{Fe}$ abundance ratios is observed both within a galaxy and from galaxy to galaxy when comparing the abundance measurements of stars in the Milky Way with those in the Magellanic Clouds and dwarf spheroidal galaxies (Venn 1999; Shetrone et al. 2003; Tolstoy et al. 2003). Thus, the observed dispersion in the DLA cloud-to-cloud abundance ratios suggests at first glance that the individual clouds do not have a similar enrichment history or a uniform differential dust depletion. Moreover, the fact that this dispersion is higher than the one of global DLA abundance measurements indicates that the SFH, dust, and ionization variations are confined more to individual clouds rather than to the whole DLA galaxy. If confirmed, this will provide important constraints on the understanding of the ISM of high-redshift galaxies and the enrichment of gas in the early Universe. In Sect. 6 we study the cloud-to-cloud variations further within a given DLA system.

In the second part of Table 10, we present the results of our study on different possible correlations between an abundance ratio $[\mathrm{X} / \mathrm{Y}]$ and $[\mathrm{Zn} / \mathrm{Fe}]$. The $[\mathrm{Zn} / \mathrm{Fe}]$ ratio is considered as a

\footnotetext{
${ }^{4}$ The number of $\sigma$ is computed as the square root of the reduced $\chi^{2}$. This is a first order approximation of the probability to have a statistically significant dispersion assuming a Gaussian distribution.
}

dust depletion indicator (see Sect. 4). A few of these correlations have already been explored by e.g. Prochaska \& Wolfe (2002) for the abundance measurements obtained in DLAs, but this is the first time that we probe such properties for the individual clouds within DLAs. The trend discussed above that $\mathrm{SFH} /$ dust/ionization variations are likely to be more confined to individual clouds than to the global DLA galaxies suggests that if some correlations exist between some physical properties, they should be more easily identified in individual cloud studies. We searched for correlations between $[\mathrm{Zn} / \mathrm{Fe}]$ and the nucleosynthesis indicators [Si/Fe], [S/Fe], [Si/Zn], [S/Zn], [N/Si], and $[\mathrm{Mn} / \mathrm{Fe}]$, and between $[\mathrm{Zn} / \mathrm{Fe}]$ and other dust depletion indicators $[\mathrm{S} / \mathrm{Si}]$ and $[\mathrm{Cr} / \mathrm{Fe}]$. A Kendall test was performed to analyze these correlations, and in Col. (7) we give the Kendall correlation factor $\tau$ and in Col. (8) the probability $\mathrm{P}(\tau)$ under the null hypothesis of zero correlation (values lower than $5 \%$ indicate a significant correlation). In Cols. (9) and (10), we report the zero points and slopes with their $1 \sigma$ errors of the linear least-square regressions, $[\mathrm{X} / \mathrm{Y}]=a+b \times[\mathrm{Zn} / \mathrm{Fe}]$, computed for the cases of significant correlations. The errors on both $[\mathrm{X} / \mathrm{Y}]$ and $[\mathrm{Zn} / \mathrm{Fe}]$ ratios were taken into account in this computation. We have not, however, accounted for the fact that $\mathrm{Zn}$ or Fe are generally present in the ratios along each axis (i.e. $[\mathrm{Zn} / \mathrm{Fe}]$ and $[\mathrm{X} / \mathrm{Y}])$. Therefore, one should be more skeptical of correlations with less than $99 \%$ significance.

Figure 16 shows that there are clear correlations between $[\mathrm{Si} / \mathrm{Fe}]$ and $[\mathrm{S} / \mathrm{Fe}]$ versus $[\mathrm{Zn} / \mathrm{Fe}]$ and clear anti-correlations between $[\mathrm{Si} / \mathrm{Zn}]$ and $[\mathrm{S} / \mathrm{Zn}]$ versus $[\mathrm{Zn} / \mathrm{Fe}]$. These trends are the result of a combination of dust depletion and nucleosynthesis enrichment effects. But, more precisely, the increase of the $[\alpha / \mathrm{Fe}]$ ratios and the decrease of the $[\mathrm{Si} / \mathrm{Zn}]$ ratio with the dust depletion level can mainly be assigned to differential dust depletion effects. The nucleosynthesis enrichment effects contribute only negligibly to the strength of the evolution as a function of dust depletion, as illustrated by [S/Zn] versus 

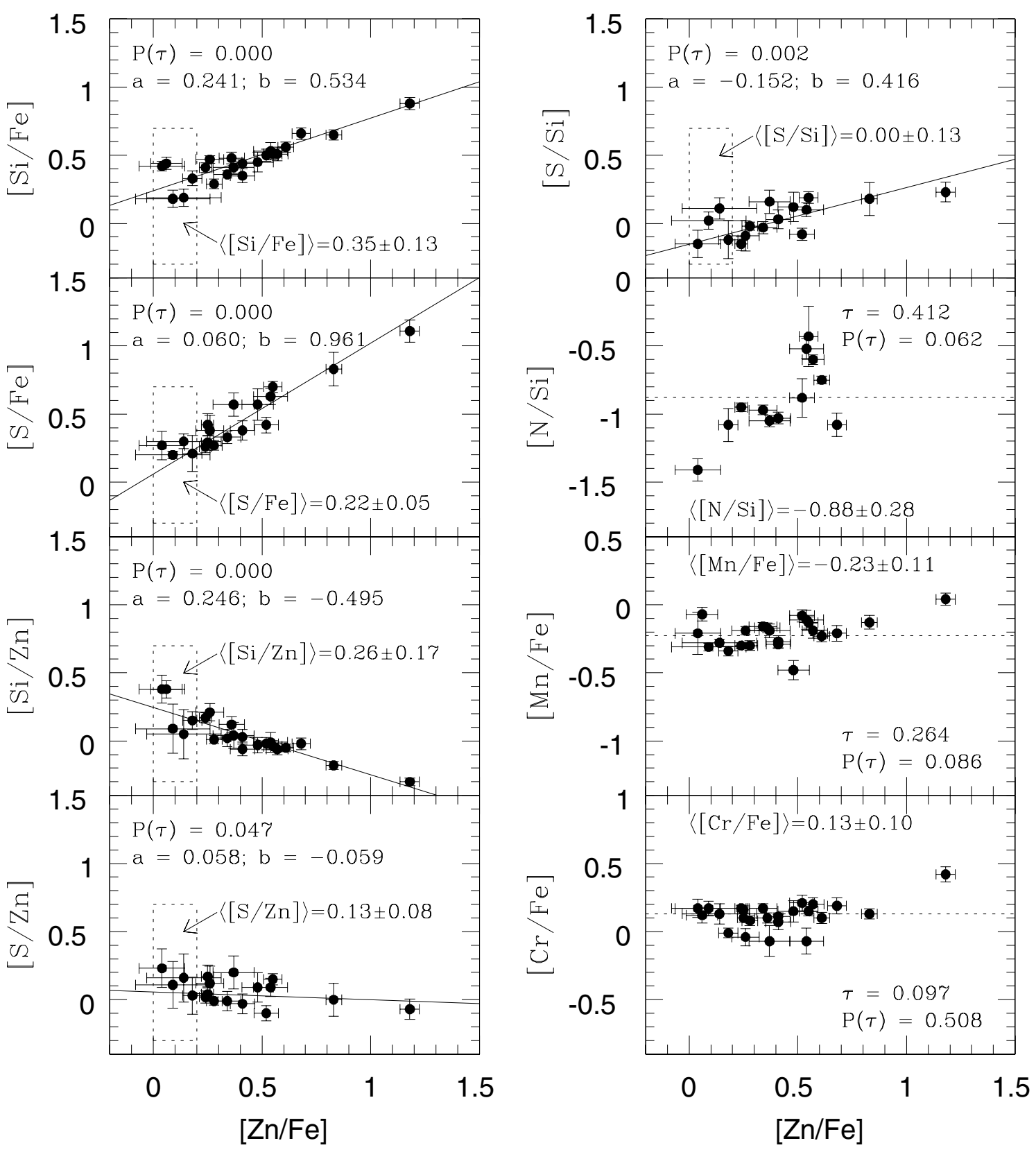

Fig. 16. Eight different abundance ratios $[\mathrm{X} / \mathrm{Y}]$ versus $[\mathrm{Zn} / \mathrm{Fe}]$ for the entire set of clouds observed in our sample of 11 DLAs. The Kendall test shows clear correlations between $[\mathrm{Si} / \mathrm{Fe}],[\mathrm{S} / \mathrm{Fe}],[\mathrm{Si} / \mathrm{Zn}],[\mathrm{S} / \mathrm{Zn}],[\mathrm{S} / \mathrm{Si}]$, and $[\mathrm{Zn} / \mathrm{Fe}]$ with a probability under the null hypothesis of zero correlation, $P(\tau),<5 \%$. The derived linear least-square regressions, $[\mathrm{X} / \mathrm{Y}]=a+b \times[\mathrm{Zn} / \mathrm{Fe}]$, computed by taking into account the errors on both $[\mathrm{X} / \mathrm{Y}]$ and $[\mathrm{Zn} / \mathrm{Fe}]$ data points, are shown by the solid lines. The dotted horizontal lines correspond to the weighted mean of $[\mathrm{X} / \mathrm{Y}]$ and are indicated when no correlation is observed. In panels 1-5 (from top to bottom and from left to right), we note the weighted mean of [X/Y] in the interval $0<[\mathrm{Zn} / \mathrm{Fe}]<0.2$ (data points contained in the dotted boxes).

$[\mathrm{Zn} / \mathrm{Fe}]$. Indeed, the $[\mathrm{S} / \mathrm{Zn}]$ ratio, which is independent of dust depletion effects, is an intrinsic tracer of nucleosynthesis enrichment, and the correlation between $[\mathrm{S} / \mathrm{Zn}]$ and $[\mathrm{Zn} / \mathrm{Fe}]$ is observed at only $95 \%$ confidence level, and the slope of its linear least-square regression is consistent with zero $(b=-0.059 \pm$ $0.071)$. This analysis, in addition, suggests that the high dispersion observed in the $[\mathrm{Si} / \mathrm{Fe}],[\mathrm{S} / \mathrm{Fe}]$, and $[\mathrm{Si} / \mathrm{Zn}]$ ratios (see the first part of Table 10) is the result of only dust depletion effects. The nucleosynthesis enrichment effects are, indeed, negligible given the low dispersion measured in the $[\mathrm{S} / \mathrm{Zn}]$ ratio and the low dispersion of $[\mathrm{Si} / \mathrm{Fe}],[\mathrm{S} / \mathrm{Fe}]$, and $[\mathrm{Si} / \mathrm{Zn}]$ along the linear least-square regressions (see Fig. 16). Consequently, the individual clouds very likely have a similar enrichment history, but different dust depletion levels.

The signature of pure nucleosynthesis contribution (i.e. of the SFH) can be observed in the values of the $[\alpha / \mathrm{Fe}, \mathrm{Zn}]$ ratios at $[\mathrm{Zn} / \mathrm{Fe}] \simeq 0$. Indeed, at $[\mathrm{Zn} / \mathrm{Fe}] \simeq 0$, the dust depletion level is low, and hence the $\alpha$-element over iron-peak element ratios are free from dust depletion effects. We computed the weighted means of data points contained in the interval $[\mathrm{Zn} / \mathrm{Fe}]=[0,+0.2]$ (see the dotted boxes in Fig. 16), and interestingly we find enhanced $[\alpha / \mathrm{Fe}, \mathrm{Zn}]$ ratios relative to solar 
in all cases: $\langle[\mathrm{Si} / \mathrm{Fe}]\rangle=+0.35 \pm 0.13,\langle[\mathrm{~S} / \mathrm{Fe}]\rangle=+0.22 \pm 0.05$, $\langle[\mathrm{Si} / \mathrm{Zn}]\rangle=+0.26 \pm 0.17$, and $\langle[\mathrm{S} / \mathrm{Zn}]\rangle=+0.13 \pm 0.08$. This suggests that the intrinsic abundance patterns of individual clouds within DLAs, when the nucleosynthesis enrichment can reliably be disentangled from dust depletion effects, show an $\alpha$-enhancement indicative of type II SNe. We would like, in addition, to underline the relatively important difference which exists between the mean $[\mathrm{Si} / \mathrm{Fe}]$ ratio and the mean $[\mathrm{S} / \mathrm{Zn}]$ ratio, yet measured in the same clouds free from dust depletion effects. The fact that $[\mathrm{S} / \mathrm{Zn}]$ is almost solar, while $[\mathrm{Si} / \mathrm{Fe}]$ shows a clear $\alpha$-enhancement, suggests that the $[\mathrm{S} / \mathrm{Zn}]$ ratio, although independent from dust depletion effects, may not be a reliable tracer of nucleosynthesis enrichment as considered until now (e.g. Centurión et al. 2000). Indeed, the S production is perhaps correlated in some way with the production of $\mathrm{Zn}$ (see Fenner et al. 2004).

Some of the correlations and anti-correlations found in this analysis of clouds within DLAs have already been observed in DLAs themselves (e.g. [Si/Fe] versus $[\mathrm{Zn} / \mathrm{Fe}]$ ), while for some we observe only trends due to fewer data points. Similarly, the $\alpha$-enhanced abundance pattern unambiguously observed in individual clouds is still being debated for the global DLA abundance patterns (Prochaska \& Wolfe 2002; Vladilo 1998, 2002; Centurión et al. 2000). We stressed this issue in Sect. 4 with the mean DLA $[\mathrm{Si}, \mathrm{S} / \mathrm{Fe}]$ ratios showing an $\alpha$-enhancement and the mean $[\mathrm{Si} / \mathrm{Zn}]$ ratios showing solar values.

We also explored the correlations of $[\mathrm{Zn} / \mathrm{Fe}]$ with two other nucleosynthesis enrichment indicators, $[\mathrm{N} / \mathrm{Si}]$ and $[\mathrm{Mn} / \mathrm{Fe}]$. No clear correlation was observed for any of these ratios (see Fig. 16). This is particularly interesting in the case of $[\mathrm{Mn} / \mathrm{Fe}]$, since it shows that all the clouds have an undersolar $[\mathrm{Mn} / \mathrm{Fe}]$ ratio, irrespective of dust depletion level, except for the dustiest cloud with $[\mathrm{Zn} / \mathrm{Fe}]>+1$. Hence, this underabundance is only a result of nucleosynthesis enrichment. Evidence of intrinsic subsolar $[\mathrm{Mn} / \mathrm{Fe}]$ abundances in DLAs themselves were discussed in Sect. 4.

Finally, we found an interesting correlation between [Zn/Fe] and the ratio of two $\alpha$-elements: S, a volatile element, and $\mathrm{Si}$, a refractory element (see Fig. 16). This correlation is a direct result of pure differential dust depletion effects observed in two $\alpha$-elements and two iron-peak elements. The mean value of the $[\mathrm{S} / \mathrm{Si}]$ ratio in the interval $[\mathrm{Zn} / \mathrm{Fe}]=[0,+0.2]$ is solar, $\langle[\mathrm{S} / \mathrm{Si}]\rangle=0.00 \pm 0.13$, which shows that $\mathrm{S}$ traces $\mathrm{Si}$ in the presence of a weak dust depletion level.

\section{Cloud-to-cloud chemical variations in individual DLA systems}

Having analyzed the cloud-to-cloud variations of the entire set of clouds observed in our sample of 11 DLA systems, we would now like to discuss the cloud-to-cloud chemical variations in individual DLAs in more detail. We apply the same definition of a cloud as in Sect. 5. Prochaska \& Wolfe (1996) were the first to quantitatively investigate variations in the chemical abundances of a single DLA. They compared ionic column densities along the observed velocity profile using the apparent optical depth method (Savage \& Sembach 1991). Then, Lopez et al. (2002) were the first to present a detailed cloud-to-cloud analysis for a $z>2$ damped system based on a Voigt profile analysis.
And more recently, Prochaska (2003) performed the chemical abundance variation analysis along the sightlines of 13 DLAs. Together, these studies argue that the majority of DLAs have very uniform relative abundances. In Sect. 5 we have, however, shown that a high dispersion is observed when all the clouds of different DLAs are studied together.

There are three possible sources of abundance variations among clouds: (i) different nucleosynthesis enrichments, i.e. different star formation histories from cloud-to-cloud; (ii) different dust depletion levels, i.e. different dust amounts and/or physical conditions from cloud-to-cloud; and (iii) different ionization conditions, i.e. different densities (self-shielding) and/or ionizing fluxes from cloud-to-cloud. Each of these three sources can be tested with specific abundance ratios (see Sect. 1). Analyzing these specific abundance ratios in a given DLA galaxy, we tried to determine the sources leading to cloud-to-cloud variations within a DLA galaxy.

In Table 11 we describe the different abundance ratios analyzed and the number of clouds observed within each DLA. $[\mathrm{Si} / \mathrm{Fe}]$ is the only abundance ratio for which a measurement is obtained in all the clouds along a given QSO sightline. In Cols. (4)-(6) we give the first simple statistical results for the cloud-to-cloud abundance variations, namely the logarithmic weighted mean computed using the $1 \sigma$ errors as weights, the logarithmic rms dispersion, and the reduced $\chi^{2}$ relative to the weighted mean, respectively. In addition, we performed a series of Monte-Carlo simulations, using the technique designed by Prochaska (2003), to investigate the deviations allowed within each DLA and to derive quantitative limits and values of the cloud-to-cloud variations relative to the weighted mean. This technique consists of making three measures of the uniformity within individual DLAs. With one measure, we study an extreme scenario where all of the variation arises from a single cloud. In Col. (7) we give the values of $\Delta_{\text {sngl }}$, the minimum $[\mathrm{X} / \mathrm{Y}]$ variation in a single cloud, which gives $\chi_{\mathrm{M}-\mathrm{C}}^{2}>\chi_{\mathrm{obs}}^{2}$ in over $95 \%$ of the 1000 trials in the MonteCarlo analysis. The two other measures assume deviations in all the clouds with values drawn from a uniform distribution. In Cols. (8) and (9) we calculate, respectively, $\Delta_{\text {all }}$, the minimum variation which when applied to every cloud gives $\chi_{\mathrm{M}-\mathrm{C}}^{2}>\chi_{\mathrm{obs}}^{2}$ in over $95 \%$ of the trials, and $\Delta_{\text {best }}$, the variation which when applied to each cloud has the highest probability of yielding $\chi^{2}=\chi_{\text {obs }}^{2} \pm 10 \%$. The $\Delta_{\text {all }}$ values are the most realistic upper limits to abundance variations and $\Delta_{\text {best }}$ reflects the most likely value (for more details, see Prochaska 2003).

We performed the cloud-to-cloud chemical variation analysis only in the DLA systems in which the majority of the abundance ratios considered can be measured in at least three clouds. For this reason, the DLAs toward Q0841+129, Q2348-1444, and Q0100+13 were excluded. We now briefly discuss the cloud-to-cloud abundance ratios for the seven remaining DLAs studied. For each analyzed DLA, we plot the cloud-to-cloud abundance ratios as a function of the velocity of the clouds.

DLA toward Q0450-13. Figure 17 shows the cloud-to-cloud abundance ratios of this DLA. A large variation, larger than 0.2 dex, was observed in all the relative abundances analyzed in this DLA (see Table 11). The most impressive is the 
Table 11. Cloud-to-cloud chemical variation analysis in individual DLA systems.

\begin{tabular}{|c|c|c|c|c|c|c|c|c|}
\hline$[\mathrm{X} / \mathrm{Y}]$ & ${ }^{\# \text { clouds }^{a}}$ & $\overline{\Delta v^{b}}$ & Mean & rms & $\overline{\chi_{v}^{2}}$ & $\Delta_{\text {sngl }}$ & $\Delta_{\text {all }}$ & $\Delta_{\text {best }}$ \\
\hline \multicolumn{9}{|c|}{ DLA toward Q0450-13 } \\
\hline$[\mathrm{Si} / \mathrm{Fe}]$ & 11 & 232 & 0.313 & 0.151 & 13.66 & 0.69 & 0.34 & 0.21 \\
\hline$[\mathrm{O} / \mathrm{Si}]$ & 7 & 232 & -0.345 & 0.636 & 117.36 & 1.28 & 1.47 & 0.75 \\
\hline$[\mathrm{S} / \mathrm{Si}]$ & 6 & 86 & 0.038 & 0.172 & 3.51 & 0.46 & 0.39 & 0.18 \\
\hline$[\mathrm{Mg} / \mathrm{Si}]$ & 6 & 86 & 1.103 & 0.227 & 6.57 & 0.54 & 0.51 & 0.27 \\
\hline$[\mathrm{N} / \mathrm{Si}]$ & 7 & 138 & -1.275 & 0.282 & 12.82 & 0.69 & 0.58 & 0.29 \\
\hline$[\mathrm{N} / \mathrm{S}]$ & 6 & 86 & -1.311 & 0.173 & 4.68 & 0.54 & 0.50 & 0.25 \\
\hline \multicolumn{9}{|c|}{ DLA toward Q1157+014 } \\
\hline$[\mathrm{Si} / \mathrm{Fe}]$ & 4 & 144 & 0.448 & 0.026 & 0.26 & 0.07 & 0.06 & 0.00 \\
\hline$[\mathrm{Si} / \mathrm{Zn}]$ & 3 & 72 & 0.158 & 0.182 & 9.49 & 0.42 & 1.31 & 0.27 \\
\hline$[\mathrm{Mg} / \mathrm{Si}]$ & 4 & 144 & -0.010 & 0.049 & 0.61 & 0.15 & 0.16 & 0.00 \\
\hline$[\mathrm{Mn} / \mathrm{Fe}]$ & 4 & 144 & -0.214 & 0.095 & 5.74 & 0.31 & 0.44 & 0.14 \\
\hline$[\mathrm{Zn} / \mathrm{Fe}]$ & 3 & 72 & 0.311 & 0.201 & 8.26 & 0.41 & 1.21 & 0.26 \\
\hline \multicolumn{9}{|c|}{ DLA toward Q1210+17 } \\
\hline$[\mathrm{Si} / \mathrm{Fe}]$ & 6 & 127 & 0.204 & 0.058 & 2.68 & 0.26 & 0.20 & 0.09 \\
\hline$[\mathrm{Si} / \mathrm{Zn}]$ & 3 & 51 & 0.013 & 0.060 & 0.12 & 0.00 & 0.01 & 0.07 \\
\hline$[\mathrm{S} / \mathrm{Zn}]$ & 3 & 51 & -0.003 & 0.139 & 0.67 & 0.29 & 0.37 & 0.07 \\
\hline$[\mathrm{S} / \mathrm{Si}]$ & 3 & 51 & -0.001 & 0.080 & 1.29 & 0.19 & 0.37 & 0.00 \\
\hline$[\mathrm{Mn} / \mathrm{Fe}]$ & 3 & 51 & -0.296 & 0.015 & 0.29 & 0.07 & 0.10 & 0.03 \\
\hline$[\mathrm{Zn} / \mathrm{Fe}]$ & 3 & 51 & 0.267 & 0.156 & 0.88 & 0.33 & 0.45 & 0.00 \\
\hline \multicolumn{9}{|c|}{ DLA toward $1331+17^{1}$} \\
\hline$[\mathrm{Si} / \mathrm{Fe}]$ & 4 & 89 & 0.595 & 0.200 & 23.02 & 0.48 & 0.85 & 0.29 \\
\hline$[\mathrm{Si} / \mathrm{Zn}]$ & 4 & 89 & -0.182 & 0.238 & 21.82 & 0.46 & 0.79 & 0.27 \\
\hline$[\mathrm{S} / \mathrm{Zn}]$ & 4 & 89 & 0.005 & 0.090 & 0.85 & 0.32 & 0.40 & 0.02 \\
\hline$[\mathrm{S} / \mathrm{Si}]$ & 4 & 89 & 0.139 & 0.149 & 2.00 & 0.44 & 0.59 & 0.18 \\
\hline$[\mathrm{Mg} / \mathrm{Si}]$ & 4 & 89 & 0.199 & 0.167 & 0.31 & 0.51 & 0.53 & 0.05 \\
\hline$[\mathrm{Mn} / \mathrm{Fe}]$ & 4 & 89 & -0.154 & 0.226 & 13.99 & 0.43 & 0.77 & 0.22 \\
\hline$[\mathrm{Zn} / \mathrm{Fe}]$ & 4 & 89 & 0.810 & 0.476 & 60.47 & 0.81 & 1.45 & 0.59 \\
\hline$[\mathrm{Cr} / \mathrm{Fe}]$ & 4 & 89 & 0.164 & 0.192 & 10.94 & 0.46 & 0.82 & 0.26 \\
\hline \multicolumn{9}{|c|}{ DLA toward Q2230+02 } \\
\hline$[\mathrm{Si} / \mathrm{Fe}]$ & 16 & 401 & 0.408 & 0.078 & 3.02 & 0.55 & 0.19 & 0.12 \\
\hline$[\mathrm{Si} / \mathrm{Zn}]$ & 4 & 144 & -0.019 & 0.047 & 0.48 & 0.15 & 0.14 & 0.02 \\
\hline$[\mathrm{S} / \mathrm{Zn}]$ & 4 & 144 & -0.033 & 0.137 & 1.77 & 0.32 & 0.39 & 0.10 \\
\hline$[\mathrm{O} / \mathrm{Si}]$ & 4 & 370 & -0.593 & 0.303 & 10.27 & 0.72 & 1.31 & 0.43 \\
\hline$[\mathrm{S} / \mathrm{Si}]$ & 6 & 144 & -0.011 & 0.101 & 1.63 & 0.36 & 0.26 & 0.09 \\
\hline$[\mathrm{N} / \mathrm{Si}]$ & 7 & 161 & -1.003 & 0.122 & 1.75 & 0.33 & 0.20 & 0.07 \\
\hline$[\mathrm{N} / \mathrm{S}]$ & 6 & 144 & -1.021 & 0.202 & 2.50 & 0.55 & 0.44 & 0.18 \\
\hline$[\mathrm{Mn} / \mathrm{Fe}]$ & 6 & 161 & -0.155 & 0.087 & 3.09 & 0.32 & 0.25 & 0.10 \\
\hline$[\mathrm{Zn} / \mathrm{Fe}]$ & 4 & 144 & 0.423 & 0.082 & 1.69 & 0.28 & 0.35 & 0.08 \\
\hline \multicolumn{9}{|c|}{ DLA toward Q2231-00 ${ }^{1}$} \\
\hline$[\mathrm{Si} / \mathrm{Fe}]$ & 10 & 161 & 0.349 & 0.136 & 7.19 & 0.61 & 0.32 & 0.18 \\
\hline$[\mathrm{Si} / \mathrm{Zn}]$ & 4 & 123 & 0.015 & 0.093 & 2.16 & 0.36 & 0.41 & 0.10 \\
\hline$[\mathrm{S} / \mathrm{Zn}]$ & 4 & 123 & 0.132 & 0.177 & 0.89 & 0.33 & 0.29 & 0.08 \\
\hline$[\mathrm{S} / \mathrm{Si}]$ & 8 & 135 & 0.233 & 0.259 & 5.83 & 0.89 & 0.57 & 0.26 \\
\hline$[\mathrm{Mn} / \mathrm{Fe}]$ & 5 & 135 & -0.216 & 0.097 & 5.02 & 0.38 & 0.41 & 0.16 \\
\hline$[\mathrm{Zn} / \mathrm{Fe}]$ & 4 & 123 & 0.391 & 0.231 & 14.45 & 0.62 & 1.04 & 0.30 \\
\hline$[\mathrm{Cr} / \mathrm{Fe}]$ & 5 & 135 & 0.066 & 0.110 & 3.43 & 0.39 & 0.39 & 0.12 \\
\hline \multicolumn{9}{|c|}{ DLA toward Q2343+12 } \\
\hline$[\mathrm{Si} / \mathrm{Fe}]$ & 19 & 355 & 0.515 & 0.208 & 24.37 & 1.09 & 0.45 & 0.33 \\
\hline$[\mathrm{Si} / \mathrm{Zn}]$ & 3 & 35 & -0.045 & 0.021 & 0.25 & 0.08 & 0.12 & 0.00 \\
\hline$[\mathrm{O} / \mathrm{Si}]$ & 17 & 305 & -0.153 & 0.265 & 20.86 & 1.12 & 0.52 & 0.37 \\
\hline$[\mathrm{N} / \mathrm{Si}]$ & 9 & 305 & -0.810 & 0.465 & 18.54 & 1.00 & 0.77 & 0.36 \\
\hline$[\mathrm{Mn} / \mathrm{Fe}]$ & 3 & 35 & -0.214 & 0.021 & 0.16 & 0.10 & 0.12 & 0.00 \\
\hline$[\mathrm{Zn} / \mathrm{Fe}]$ & 3 & 35 & 0.623 & 0.056 & 1.53 & 0.17 & 0.39 & 0.06 \\
\hline
\end{tabular}

${ }^{1}$ DLAs from our first sample, see their metal line profiles in Paper I.

${ }^{a}$ Number of clouds in a given DLA with a column density measurement for both the elements $\mathrm{X}$ and $\mathrm{Y}$.

${ }^{b}$ Velocity range in $\mathrm{km} \mathrm{s}^{-1}$ covered by the clouds in the DLA galaxy for a given [X/Y] ratio. 


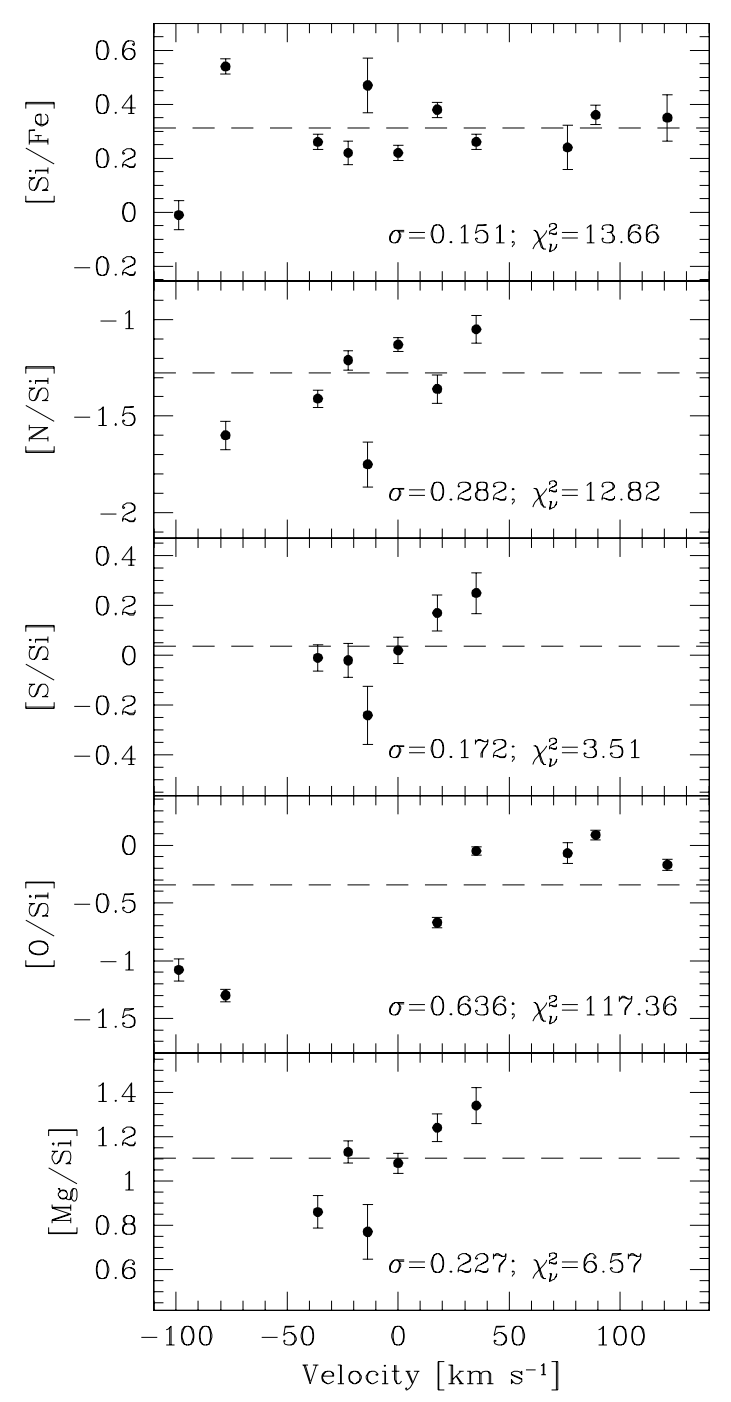

Fig. 17. Cloud-to-cloud $[\mathrm{X} / \mathrm{Y}]$ abundance ratios in the DLA toward Q0450-13. A total number of 11 clouds covering $232 \mathrm{~km} \mathrm{~s}^{-1}$ in velocity space are detected in this DLA along the QSO line of sight. The dashed line corresponds to the logarithmic weighted mean of the cloud-to-cloud $[\mathrm{X} / \mathrm{Y}]$ ratios computed by using their $1 \sigma$ errors as weights. $\sigma$ indicates the logarithmic rms dispersion in $[\mathrm{X} / \mathrm{Y}]$, and $\chi_{v}^{2}$ the reduced $\chi^{2}$ relative to the weighted mean. In Table 11 we give additional statistical information essential for an analysis of the amplitude of the cloud-to-cloud chemical variations in individual DLAs.

cloud-to-cloud variation of the $[\mathrm{O} / \mathrm{Si}]$ ratio observed at $10 \sigma$ with $\Delta_{\text {best }}=0.75$ dex. It most likely results from a high variation in the ionization level from cloud-to-cloud. In the clouds at $v>+30 \mathrm{~km} \mathrm{~s}^{-1}$, we observe solar $[\mathrm{O} / \mathrm{Si}]$ ratios and in the clouds around $-90 \mathrm{~km} \mathrm{~s}^{-1}$, the $[\mathrm{O} / \mathrm{Si}]$ ratio is highly undersolar reaching -1.5 dex and indicating a high ionization level. The presence of strong ionization signatures in this DLA was already pointed out in Sect. 3.1 with the detection of strong intermediate-ion lines, Fe III and N II. The deviation from uniformity observed in the other abundance ratios with $\Delta_{\text {best }}>$ 0.2 dex is very interesting, all the more since a similar trend of increasing abundance ratios from bluer to redder clouds is observed. The fact that this trend is particularly pronounced in the

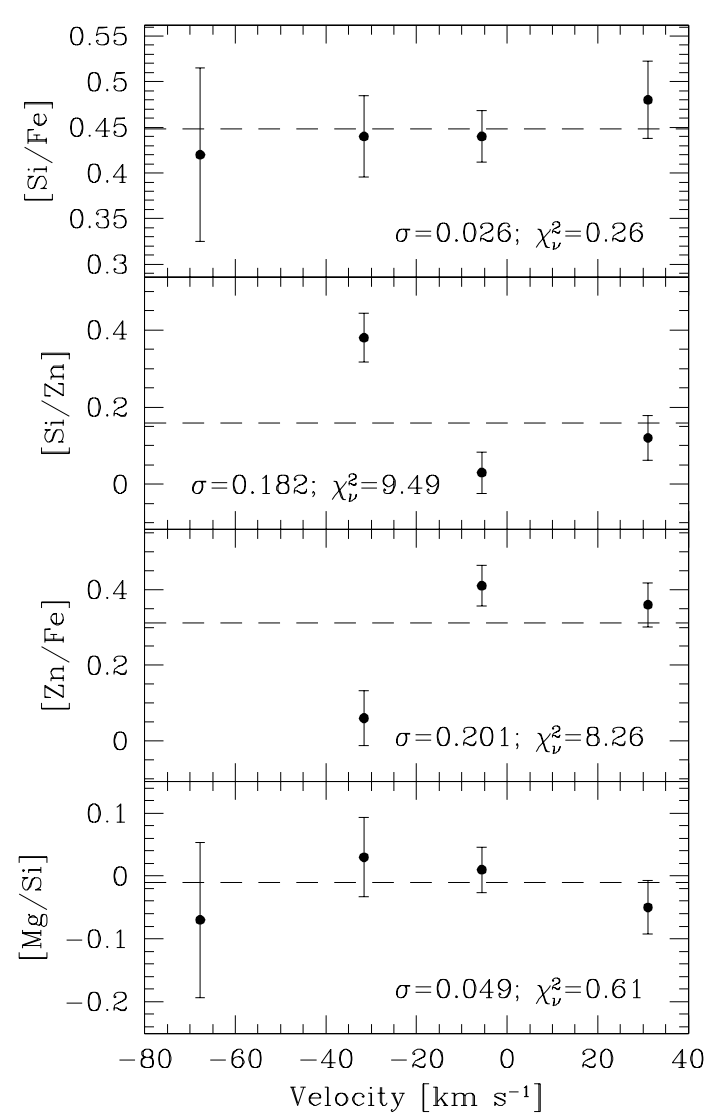

Fig. 18. Same as Fig. 17 for the DLA toward Q1157+014. A total number of 4 clouds covering $144 \mathrm{~km} \mathrm{~s}^{-1}$ in velocity space are detected in this DLA along the QSO line of sight.

[N/Si] ratio with a $3 \sigma$ variation confirms that it is due to a variation in the ionization level from cloud-to-cloud, and it provides constraints on the geometry of the ionizing flux in this DLA galaxy (see further details in Paper IV).

DLA toward Q1157+014. In Fig. 18 we show the cloud-tocloud abundance ratio plots for this DLA. The [Si/Fe] and $[\mathrm{Mg} / \mathrm{Si}]$ ratios are perfectly uniform, while $[\mathrm{Zn} / \mathrm{Fe}]$ and $[\mathrm{Si} / \mathrm{Zn}]$ show variations at the $3 \sigma$ level (see Table 11). This variation is mainly due to one cloud at $-32 \mathrm{~km} \mathrm{~s}^{-1}$ that has a lower [ $\left.\mathrm{Zn} / \mathrm{Fe}\right]$ value and a higher [Si/Zn] value than the other two clouds with $[\mathrm{Zn} / \mathrm{Fe}]$ and $[\mathrm{Si} / \mathrm{Zn}]$ measurements. A careful examination of the Zn II, Fe II, and Si II profiles of this DLA already suggests some clues for variation. Indeed, the optical depth of component 3 (corresponding to the cloud at $-32 \mathrm{~km} \mathrm{~s}^{-1}$ ) is much deeper in the Fe II and Si II lines than in the $\mathrm{Zn}$ II lines in comparison with the redder components. This variation can be assigned to a dust depletion variation from cloud-to-cloud. Indeed, the deeper Fe II and Si II optical depths in the cloud at $-32 \mathrm{~km} \mathrm{~s}^{-1}$ indicate a lower dust amount. As a consequence, the abundances of $\mathrm{Fe}$ and $\mathrm{Si}$, two refractory elements, are not depleted relative to $\mathrm{Zn}$, a volatile element, in this cloud, while they are depleted in the clouds at -5 and $+31 \mathrm{~km} \mathrm{~s}^{-1}$, in which we measure an enhanced $[\mathrm{Zn} / \mathrm{Fe}]$ ratio and a low $[\mathrm{Si} / \mathrm{Zn}]$ ratio. DLAs toward Q1210+17 and Q2230+02. For these DLAs, we do not show the cloud-to-cloud abundance ratio plots, because all their relative abundances are uniform to better than 


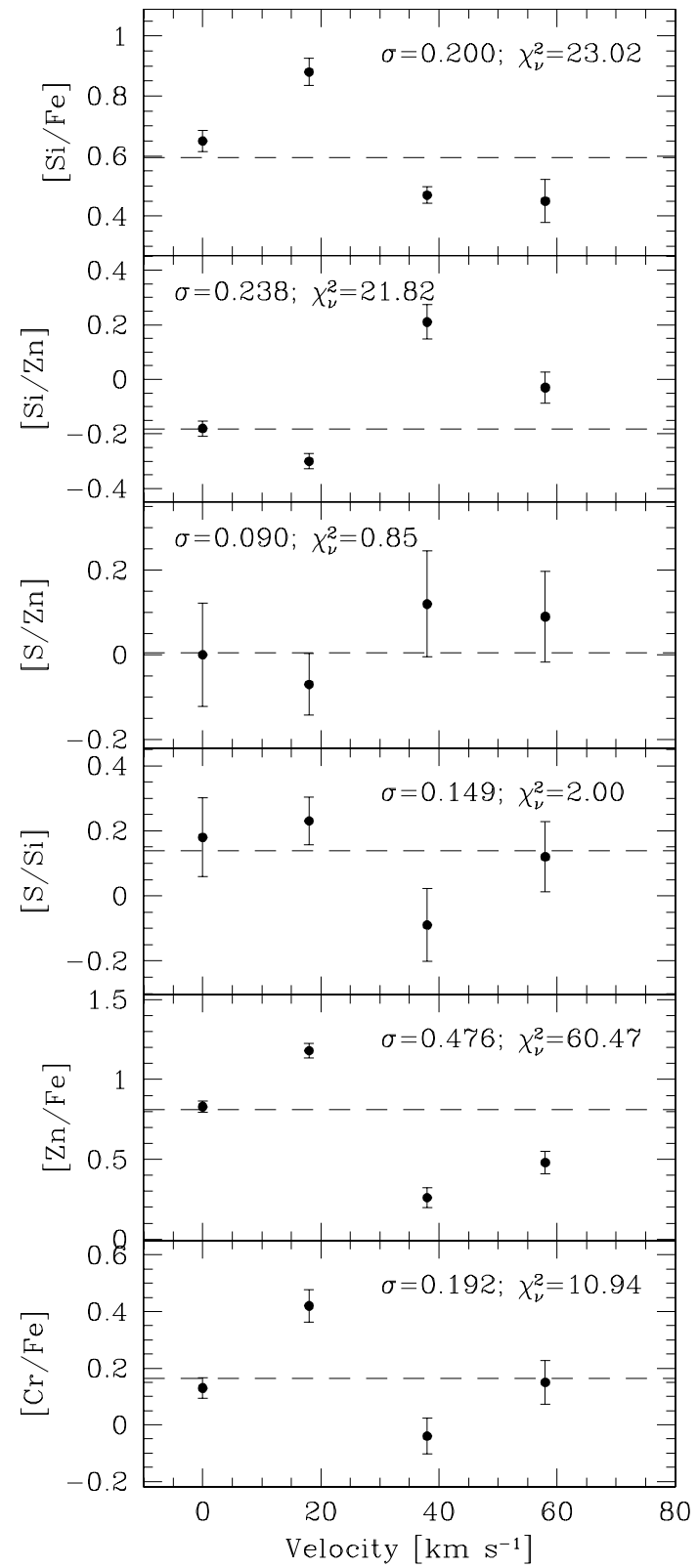

Fig. 19. Same as Fig. 17 for the DLA toward Q1331+17 from the first sample of DLAs (see Paper I). A total number of 4 clouds covering $89 \mathrm{~km} \mathrm{~s}^{-1}$ in velocity space are detected in this DLA along the QSO line of sight.

0.1 dex (see Table 11). This reveals an important characteristic of these two high-redshift galaxies: the gas clouds which comprise these galaxies apparently have very similar physical properties (mainly when considering the differential dust depletion) and nucleosynthetic enrichment histories. Only the $[\mathrm{O} / \mathrm{Si}]$ ratio in the DLA toward Q2230+02 seems to show a $3 \sigma$ departure from uniformity. This may indicate a variation in the ionization properties within this DLA galaxy, between the cloud observed at $-147 \mathrm{~km} \mathrm{~s}^{-1}$ which appears to be more ionized, and the three clouds observed around $+180 \mathrm{~km} \mathrm{~s}^{-1}$.

DLA toward Q1331+17. In Fig. 19 we show the cloudto-cloud abundance ratio plots for this DLA. A high variation in the $[\mathrm{Zn} / \mathrm{Fe}]$ ratio is observed at more than $7 \sigma$ with
$\Delta_{\text {best }}=0.59$ dex. This variation is due to differential dust depletion variations from cloud-to-cloud, with the clouds at +38 and $+58 \mathrm{~km} \mathrm{~s}^{-1}$ being less depleted (having lower $[\mathrm{Zn} / \mathrm{Fe}] \mathrm{ra}-$ tios) than the clouds at -2 and $+18 \mathrm{~km} \mathrm{~s}^{-1}$. This DLA exhibits, in fact, one of the largest dust depletion level of any DLA. The presence of a high amount of dust in the clouds at -2 and $+18 \mathrm{~km} \mathrm{~s}^{-1}$ is also favored by the detections of $\mathrm{C}^{0}$ and $\mathrm{Cl}^{0}$ in these clouds (see Fig. 4 in Paper I). Indeed, these ions are usually associated with a dense, cold neutral medium, characteristic of highly depleted gas in the Milky Way. Furthermore, they generally suggest at least a modest molecular hydrogen fraction, which is indicative of dust. This strong cloud-to-cloud dust amount variation is also responsible for variations observed in: (i) [Si/Fe], with Fe being more depleted than $\mathrm{Si}$; (ii) [Si/Zn], with $\mathrm{Si}$ being more depleted than the very mildly refractory $\mathrm{Zn}$; (iii) $[\mathrm{Cr} / \mathrm{Fe}]$, with $\mathrm{Fe}$ being more depleted than $\mathrm{Cr}$; and (iv) [S/Si], with $\mathrm{Si}$ being more depleted than the volatile S. Different SFHs from cloud-to-cloud do not seem to contribute to the $[\mathrm{Si} / \mathrm{Fe}]$ and $[\mathrm{Si} / \mathrm{Zn}]$ variations, since the $[\mathrm{S} / \mathrm{Zn}]$ ratio is perfectly uniform. Interestingly, the $[\mathrm{S} / \mathrm{Zn}]$ ratio is almost solar in all clouds, even though $[\mathrm{Si} / \mathrm{Fe}] \sim+0.4 \mathrm{dex}$ in clouds where $[\mathrm{Zn} / \mathrm{Fe}]$ is low. This joins the comment made in Sect. 5 on the use of $[\mathrm{S} / \mathrm{Zn}]$ as a nucleosynthesis enrichment indicator.

DLA toward Q2231-00. In Fig. 20 we show the cloud-tocloud abundance ratio plots for this DLA. The departures from uniformity observed in this system are modest, lower than 0.2 dex (see Table 11). Only the $[\mathrm{Zn} / \mathrm{Fe}]$ ratio seems to show a higher variation with $\Delta_{\text {best }}=0.30 \mathrm{dex}$ and may reflect some cloud-to-cloud differential dust depletion variations. However, this does not affect the uniformity of the other abundance ratios strongly. We observe only a slight effect of a higher dust depletion level in the clouds at -9 and $0 \mathrm{~km} \mathrm{~s}^{-1}$ compared to the clouds at -97 and $-72 \mathrm{~km} \mathrm{~s}^{-1}$ on the $[\mathrm{Si} / \mathrm{Fe}]$ and $[\mathrm{S} / \mathrm{Si}] \mathrm{ra}-$ tios. No sign of cloud-to-cloud SFH variations is detected, the $[\mathrm{S} / \mathrm{Zn}]$ ratio is uniform.

DLA toward Q2343+12. In Fig. 21 we show the cloud-tocloud abundance ratio plots for this DLA. The possible variations due to dust depletion and nucleosynthesis enrichment are difficult to highlight in this system, partly because many elements are detected in three clouds only (see Table 11). However, we observe high deviations from uniformity at more than $4 \sigma$ with $\Delta_{\text {best }}>0.3 \mathrm{dex}$ in the cloud-to-cloud [Si/Fe], $[\mathrm{O} / \mathrm{Si}]$, and $[\mathrm{N} / \mathrm{Si}]$ ratios. They are likely to be dominated by ionization variations. Hints of a possible trend from cloud-tocloud of higher $[\mathrm{Si} / \mathrm{Fe}]$ ratios, when $[\mathrm{O} / \mathrm{Si}]$ is lower resulting from ionization effects, can be suggested from our measurements. The presence of a high ionization level in this DLA was already pointed out in Paper I with the detection of strong intermediate-ion lines, Fe III, N II, and S III, and will be discussed further in Paper IV.

In summary, among the seven DLA systems for which we analyzed the cloud-to-cloud chemical variations, five of them do show statistically significant cloud-to-cloud variations, namely higher than $0.2 \mathrm{dex}\left(\mathrm{rms}>0.2 \mathrm{dex}\right.$ and $\left.\Delta_{\text {best }}>0.2 \mathrm{dex}\right)$ at more than $3 \sigma$, for at least two different abundance ratios. But, only two DLA systems toward Q0450-13 and Q1331+17 show "extreme" variations, that is, a higher dispersion than $0.3 \mathrm{dex}$ 


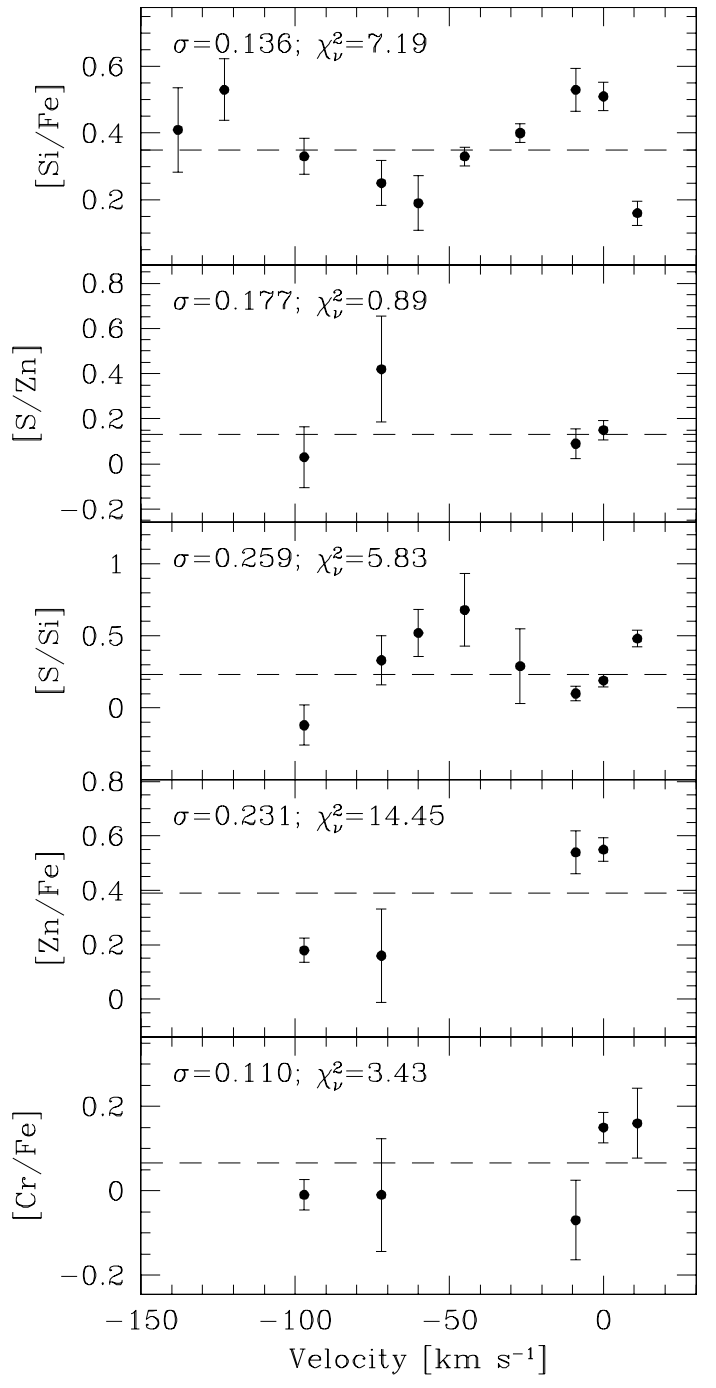

Fig. 20. Same as Fig. 17 for the DLA toward Q2231-00 from the first sample of DLAs (see Paper I). A total number of 10 clouds covering $161 \mathrm{~km} \mathrm{~s}^{-1}$ in velocity space are detected in this DLA along the QSO line of sight.

at more than $7 \sigma$. We were able to identify the sources of these variations thanks to the analysis of specific abundance ratios. These sources are either the differential dust depletion variations and/or the ionization condition variations from cloud-tocloud. But, no evidence for variations due to different SFHs was highlighted. This suggests that the gas clouds within some DLA galaxies have different physical properties, namely different dust depletion and/or ionization levels, but they all seem to show a uniform nucleosynthetic enrichment history.

In the Prochaska (2003) study based on a single abundance ratio, mainly [Si/Fe], only 2 out of 13 DLAs present cloud-tocloud variations. Our work already shows a less uniform picture for the ISM of high-redshift DLA galaxies, since 5 out of 7 DLAs show chemical variations, and all DLAs except one have at least a high $\Delta_{\text {sngl }}(>0.2$ dex $)$ for one abundance ratio indicating that there is at least a single cloud with an "abnormal" abundance. The main difference between these two studies comes from the fact that in our chemical variation analysis we considered other abundance ratios than $[\mathrm{Si} / \mathrm{Fe}]$ alone. Given

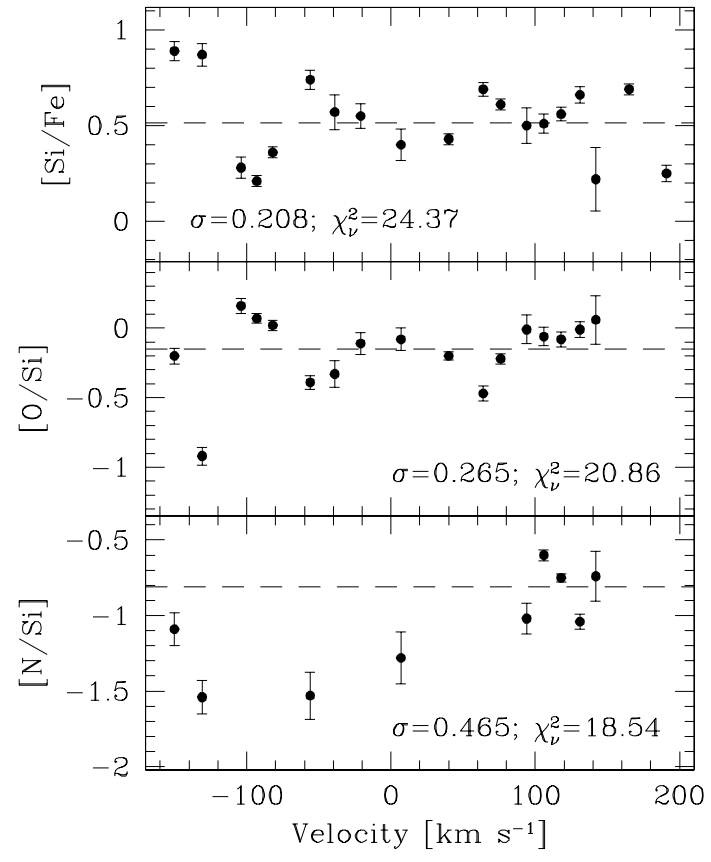

Fig. 21. Same as Fig. 17 for the DLA toward Q2343+12 from the first sample of DLAs (see Paper I). A total number of 19 clouds covering $355 \mathrm{~km} \mathrm{~s}^{-1}$ in velocity space are detected in this DLA along the QSO line of sight.

that the observed variations seem to mainly be due to dust depletion and ionization effects, as stated above, they are thus well-detected in the ratios tracing the dust depletion variations, i.e. $[\mathrm{Zn} / \mathrm{Fe}]$, and the ionization variations. Consideration of the $[\mathrm{Si} / \mathrm{Fe}]$ ratio alone would have shown variations in only 3 out of 7 DLAs, so that we would have missed variations in 2 DLA galaxies.

\section{Summary and concluding remarks}

Analysis of our sample of damped Ly $\alpha$ systems has proved once again that these systems constitute the best laboratory for studying the chemical abundances and the interstellar medium properties of high redshift galaxies. We obtained new comprehensive sets of elemental abundances of seven DLAs in the redshift range $z_{\mathrm{abs}}=1.8-2.5$ toward bright quasars. These were derived from UVES/VLT spectra combined with existing HIRES/Keck spectra. We detected 54 metal-line transitions, and obtained the column density measurements of 30 ions from 22 elements, - B, C, N, O, Mg, Al, Si, P, S, Cl, Ar, Ti, Cr, Mn, $\mathrm{Fe}, \mathrm{Co}, \mathrm{Ni}, \mathrm{Cu}, \mathrm{Zn}, \mathrm{Ge}, \mathrm{As}, \mathrm{Kr}$. Together with our first sample of four DLAs analyzed in Paper I, we have a sample of eleven DLA galaxies with uniquely comprehensive and homogeneous abundance measurements.

In this paper (II in the series) we were able to study the abundance patterns and the chemical variations of a wide range of elements in the interstellar medium of galaxies outside the Local Group, all for the first time. Chemical variations from DLA galaxy to DLA galaxy and from cloud to cloud in the ISM within a galaxy are expected, if the line of sight to a distant QSO samples regions with different star formation histories and different ISM conditions. This is suggested by 
observations in the Milky Way, the Small and Large Magellanic Clouds, and dwarf spheroidal galaxies which show chemical variations from galaxy to galaxy and within the galaxy when comparing their stellar and gas-phase abundance patterns. We considered three potential sources of observed abundance variations: the star formation history (nucleosynthesis enrichment), differential dust depletion, and ionization.

Our main results can be summarized as follows:

1) The abundance patterns of the integrated profiles of DLAs show relatively low rms dispersions, reaching only 2-3 times higher values than the statistical errors, for the majority of elements. This uniformity is remarkable given that the quasar sightlines cross gaseous regions with $\mathrm{H}$ I column densities covering an order of magnitude from $2 \times 10^{20}$ to $4 \times 10^{21} \mathrm{~cm}^{-2}$ and with metallicities ranging from $1 / 55$ to $1 / 5$ solar. This implies that the respective star formation histories, if ever different, have conspired to yield one set of relative abundances and that the effects of nucleosynthesis enrichment, dust depletion, and ionization are negligible. We discuss the implications of this uniformity element by element. The most interesting findings are: (i) the $[\mathrm{Si} / \mathrm{Fe}]$ and $[\mathrm{S} / \mathrm{Fe}]$ abundance ratios show an $\alpha$-enhancement irrespective of the dust depletion level of the DLAs studied; to reconcile this $\alpha$-enhancement with the solar $[\mathrm{Si} / \mathrm{Zn}]$ ratios, we suggest that the $[\mathrm{Zn} / \mathrm{Fe}]$ ratios are intrinsically oversolar (independent of dust depletion effects) as indicated by the recent measurements in Galactic metal-poor stars; (ii) all the Ar abundance measurements show a significant underabundance relative to $\mathrm{Si}$; however, the $[\mathrm{Ar} / \mathrm{Si}$ ] ratio alone cannot characterize the ionization of a DLA, since DLAs with low $[\mathrm{Ar} / \mathrm{Si}]$ ratios include cases where other ionization diagnostics imply the gas is predominantly neutral; (iii) all the Mn abundance measurements are underabundant relative to Fe irrespective of the dust depletion of the DLAs studied; they reach a plateau at -0.23 dex; and (iv) we obtained the second abundance measurement of Ge, an element beyond the iron-peak, in the metal-strong DLA toward Q1157+014, opening the way to investigation of s-process elements in DLAs.

2) From the Voigt profile fitting of metal-lines, we obtained very accurate component-to-component column density measurements. These components presumably correspond to interstellar medium clouds in the DLA galaxy on the QSO line of sight. By considering all the clouds of all the DLAs studied together, we see a statistically significant dispersion in several abundance ratios, for example about $5 \sigma$ in the $[\mathrm{Zn} / \mathrm{Fe}]$ ratios and larger than $3 \sigma$ in the $[\alpha / \mathrm{Fe}, \mathrm{Zn}]$ ratios. This indicates that the chemical variations are more confined to individual clouds within the DLA galaxies rather than to integrated profiles. If confirmed, this will provide important constraints on the understanding of the ISM of high-redshift galaxies and the enrichment of gas in the early Universe. We found unambiguous correlations between $[\mathrm{Si} / \mathrm{Fe}],[\mathrm{S} / \mathrm{Fe}]$, and $[\mathrm{S} / \mathrm{Si}]$ versus $[\mathrm{Zn} / \mathrm{Fe}]$, and anti-correlations between $[\mathrm{Si} / \mathrm{Zn}]$ and $[\mathrm{S} / \mathrm{Zn}]$ versus $[\mathrm{Zn} / \mathrm{Fe}]$. These trends are primarily the result of differential dust depletion effects, which are also responsible for the high cloud-to-cloud abundance ratio dispersion. The signature of the pure nucleosynthesis enrichment contribution can be observed in the $[\alpha / \mathrm{Fe}, \mathrm{Zn}]$ ratios at low dust depletion levels, $[\mathrm{Zn} / \mathrm{Fe}] \leq 0.2$. It is characterized by an $\alpha$-enhancement in the clouds. However, while the $[\mathrm{S} / \mathrm{Fe}],[\mathrm{S} / \mathrm{Fe}]$, and $[\mathrm{Si} / \mathrm{Zn}]$ ratios are highly $\alpha$-enhanced $(>0.2 \mathrm{dex})$, the $[\mathrm{S} / \mathrm{Zn}]$ ratio remains almost solar, suggesting that $[\mathrm{S} / \mathrm{Zn}]$ may not be a reliable tracer of nucleosynthesis enrichment, the production of $S$ being perhaps in some way correlated with the one of $\mathrm{Zn}$.

3) We analyzed several specific abundance ratios in individual DLA galaxies to try to determine the origin of cloudto-cloud chemical variations when observed in a given DLA galaxy. Study of the cloud-to-cloud chemical variations within seven individual DLA systems revealed that five of them show statistically significant variations, higher than $0.2 \mathrm{dex}$ at more than $3 \sigma$. Two of them show "extreme" variations with a dispersion higher than $0.3 \mathrm{dex}$ at more than $7 \sigma$. The sources of these variations are either the differential dust depletion and/or the ionization effects, while there is no evidence of variations due to different star formation histories. This suggests that the gas clouds within DLA galaxies have different physical properties, but they all seem to show a uniform nucleosynthetic enrichment history.

At lower redshift $\left(z_{\mathrm{abs}}<1\right)$, deep imaging shows that DLA galaxies are a heterogeneous group that exhibits a variety of morphologies and surface brightnesses. If this is also the case at high redshift, we may have expected higher dispersions in the DLA abundance ratios. The uniformity observed in the global gas-phase abundance patterns of DLAs is thus even more surprising. Perhaps we are penalized by the small number statistics or, more important, there may be fundamental differences between high and low redshift DLAs. The results by Kanekar \& Chengalur (2003) may be relevant here. Indeed, they derived estimates of the spin temperature, $T_{\mathrm{s}}$, in 24 DLAs and found that all DLAs with high spin temperatures, $T_{\mathrm{s}}>1000 \mathrm{~K}$, are identified with dwarf or low surface brightness galaxies, while DLAs with low $T_{\mathrm{s}}$ are associated with large, luminous galaxies. Interestingly, they observed that low redshift DLAs have both high and low values of $T_{\mathrm{s}}$, while high redshift DLAs $\left(z_{\mathrm{abs}}>2\right)$ have preferentially high $T_{\mathrm{s}}$. This result could help to understand the uniformity observed in the abundance ratios of DLAs in our sample.

The uniformity in the nucleosynthesis enrichment observed in the clouds within DLAs is also surprising and poses important constraints on the formation of high-redshift galaxies. Indeed, one very promising scenario that allows us to explain the DLA kinematics is within the CDM hierarchical cosmology and describes a DLA as multiple merging "clumps" bound to individual dark matter halos (e.g. Haehnelt et al. 1998; Maller et al. 2001). In that scenario, the uniformity of cloud-to-cloud abundance ratios within a DLA galaxy constrains the abundances of all of the protogalactic clumps making up a DLA system. In terms of nucleosynthesis enrichment, this implies the clumps share a similar chemical enrichment pattern. This represents a challenge for the CDM simulations, since those protogalactic clumps which do not share a common gas reservoir and which merge over a large timescale would not be expected to necessarily have a unique enrichment history and be at the same stage of chemical evolution. It remains to be demonstrated whether these clumps really do express very similar nucleosynthetic enrichment patterns with, in addition, very different dust depletions in some cases. This also places strict constraints 
on the mixing timescales of protogalaxies. Of course, access to the absolute values of metallicities of these clouds would help to confirm these statements. For this, however, we need to measure the $\mathrm{H}$ I column densities of individual clouds within a DLA, which is not possible from the current observations.

Acknowledgements. The authors wish to thank everyone working at $\mathrm{ESO} /$ Paranal for the high quality of UVES spectra obtained in service mode. M.D.-Z. is supported by the Swiss National Funds and extends special thanks to Professor A. Maeder for continuous encouragement. M.D.-Z. is grateful to the $\mathrm{UCO} /$ Lick Observatory for hosting her in Santa Cruz in September 2004, where the layout and main results of this paper were worked out. J.X.P. acknowledges support through the NSF grant AST 03-07824.

\section{References}

Ballester, P., Modigliani, A., Boitquin, O., et al. 2000, The Messenger, 101, 31

Calura, F., Matteucci, F., \& Vladilo, G. 2003, MNRAS, 340, 59

Centurión, M., Bonifacio, P., Molaro, P., \& Vladilo, G. 2000, ApJ, 536, 540

Centurión, M., Molaro, P., Vladilo, G., et al. 2003, A\&A, 403, 55

Chen, H.-W., \& Lanzetta, K. M. 2003, ApJ, 597, 706

Chen, Y. Q., Nissen, P. E., \& Zhao, G. 2004, A\&A, 425, 697

Chiappini, C., Matteucci, F., \& Meynet, G. 2003, A\&A, 410, 257

Cowan, J. J., Sneden, C., Beers, T. C., et al. 2005, ApJ, submitted

Dessauges-Zavadsky, M., Prochaska, J. X., \& D’Odorico, S. 2002, A\&A, 391, 801

Dessauges-Zavadsky, M., Calura, F., Prochaska, J. X., D’Odorico, S., \& Matteucci, F. 2004, A\&A, 416, 79 [Paper I]

D’Odorico, S., Cristiani, S., Dekker, H., et al. 2000, in SPIE 4005, Discoveries and Research Prospects from 8- to 10-Meter-Class Telescopes, ed. J. Bergeron, 121

Ellison, S. L., Ryan, S. G., \& Prochaska, J. X. 2001, MNRAS, 326, 628

Edvardsson, B., Pettersson, B., Kharrazi, M., \& Westerlund, B. 1995, A\&A, 293, 75

Fedchak, J. A., \& Lawler, J. E. 1999, ApJ, 523, 734

Fedchak, J. A., Wiese, L. M., \& Lawler, J. E. 2000, ApJ, 538, 773

Fenner, Y., Prochaska, J. X., \& Gibson, B. K. 2004, ApJ, 606, 116

Fontana, A., \& Ballerster, P. 1995, The Messenger, 80, 37

François, P., Matteucci, F., Cayrel, R., et al. 2004, A\&A, 421, 613

Grevesse, N., \& Sauval, A. J. 1998, Space Sci. Rev., 85, 161

Haehnelt, M. G., Steinmetz, M., \& Rauch, M. 1998, ApJ, 495, 647

Jaunsen, A. O., Jablonski, M., Pettersen, B. R., \& Stabell, R. 1995, A\&A, 300, 323

Kanekar, N., \& Chengalur, J. N. 2003, A\&A, 399, 857

Le Brun, V., Bergeron, J., Boissé, P., \& Deharveng, J. M. 1997, A\&A, 321,733

Ledoux, C., Petitjean, P., \& Srianand, R. 2003, MNRAS, 346, 209

Limongi, M., Straniero, O., \& Chieffi, A. 2000, ApJS, 129, 625

Lopez, S., Reimers, D., D’Odorico, S., \& Prochaska, J. X. 2002, A\&A, 385,778

Lu, L., Sargent, W. L. W., Barlow, T. A., Churchill, C. W., \& Vogt, S. S. 1996, ApJS, 107, 475

Maller, A. H., Prochaska, J. X., Somerville, R. S., \& Primack, J. R. 2001, MNRAS, 326, 1475

Matteucci, F., Raiteri, C. M., Busson, M., Gallino, R., \& Gratton, R. 1993, A\&A, 272, 421
Matteucci, F., \& Recchi, S. 2001, ApJ, 558, 351

McWilliam, A., Rich, R. M., \& Smecker-Hane, T. A. 2003, ApJ, 592, L21

Mishenina, T. V., Kovtyukh, V. V., Soubiran, C., Travaglio, C., \& Busso, M. 2002, A\&A, 396, 189

Molaro, P. 2005, Chemical Abundances and Mixing in Stars in the Milky Way and its Satellites, Springer-Verlag Series, ESO Astrophysics Symposia, ed. L. Pasquini, \& S. Randich

Morton, D. C. 1991, ApJS, 77, 119

Nestor, D. B., Rao, S. M., Turnshek, D. A., et al. 2002, Extragalactic Gas at Low Redshift, ed. J. S. Mulchaey, \& J. Stocke, APS Conf. Ser., 254, 34

Nissen, P. E., Chen, Y. Q., Asplund, M., \& Pettini, M. 2004, A\&A, 415, 993

Pettini, M., Smith, L. J., Hunstead, R. W., \& King, D. L. 1994, ApJ, 426, 79

Pettini, M., Lipman, K., \& Hunstead, R. W. 1995, ApJ, 451, 100

Pettini, M., Smith, L. J., King, D. L., \& Hunstead, R. W. 1997, ApJ, 486, 665

Pettini, M., Ellison, S. L., Bergeron, J., \& Petitjean, P. 2002, A\&A, 391, 21

Prochaska, J. X. 2003, ApJ, 582, 49

Prochaska, J. X., \& Wolfe, A. M. 1996, ApJ, 470, 403

Prochaska, J. X., \& Wolfe, A. M. 1999, ApJS, 121, 369

Prochaska, J. X., \& McWilliam, A. 2000, ApJ, 537, L57

Prochaska, J. X., \& Wolfe, A. M. 2002, ApJ, 566, 68

Prochaska, J. X., Wolfe, A. M., Tytler, D., et al. 2001, ApJS, 137, 21

Prochaska, J. X., Howk, J. C., O’Meara, J. M., et al. 2002a, ApJ, 571, 693

Prochaska, J. X, Henry, R. B. C., O’Meara, J. M., et al. 2002b, PASP, 114, 933

Prochaska, J. X., Howk, J. C., \& Wolfe, A. M. 2003, Nature, 423, 57

Rao, S. M., Nestor, D. B., Turnshek, D. A., et al. 2003, ApJ, 595, 94

Rao, S. M., Prochaska, J. X., Howk, J. C., \& Wolfe, A. M. 2005, AJ, 129,9

Savage, B. D., \& Sembach, K. R. 1991, ApJ, 379, 245

Savage, B. D., \& Sembach, K. R. 1996, ARA\&A, 34, 279

Shetrone, M., Venn, K. A., Tolstoy, E., et al. 2003, AJ, 125, 684

Sofia, U. J., \& Jenkins, E. B. 1998, ApJ, 499, 951

Srianand, R., Petitjean, P., \& Ledoux, C. 2000, Nature, 408, 931

Storrie-Lombardi, L. J., \& Wolfe, A. M. 2000, ApJ, 543, 552

Tolstoy, E., Venn, K. A., Shetrone, M., et al. 2003, AJ, 125, 707

Venn, K. A. 1999, ApJ, 518, 405

Viegas, S. M. 1995, MNRAS, 276, 268

Vladilo, G. 1998, ApJ, 493, 583

Vladilo, G. 2002, A\&A, 391, 407

Vladilo, G., Centurión, M., Bonifacio, P., \& Howk, J. C. 2001, ApJ, 557, 1007

Vladilo, G., Centurión, M., D’Odorico, V., \& Péroux, C. 2003, A\&A, 402, 487

Welty, D. E., Frisch, P. C., Sonneborn, G., \& York, D. G. 1999, ApJ, 512, 636

Welty, D. E., Lauroesch, J. T., Blades, J. C., Hobbs, L. M., \& York, D. G. 2001, ApJ, 554, 75

Wolfe, A. M., \& Briggs, F. H. 1981, ApJ, 248, 460

Wolfe, A. M., Turnshek, D. A., Smith, H. E., \& Cohen, R. D. 1986, ApJ, 61, 249

Wolfe, A. M., Lanzetta, K. M., Foltz, C. B., \& Chaffee, F. H. 1995, ApJ, 454, 698

Woosley, S. E., \& Weaver, T. A. 1995, ApJS, 101, 181 


\section{Online Material}


M. Dessauges-Zavadsky et al.: Data analysis and chemical variation studies, Online Material $p 2$

Table 2. Component structure of the $z_{\mathrm{abs}}=2.067$ DLA system toward Q0450-13.

\begin{tabular}{|c|c|c|c|c|c|c|c|c|c|c|c|}
\hline Comp. & $z_{\mathrm{abs}}$ & $\begin{array}{c}v_{\mathrm{rel}}^{*} \\
{\left[\mathrm{~km} \mathrm{~s}^{-1}\right]}\end{array}$ & $\begin{array}{c}b\left(\sigma_{b}\right) \\
{\left[\mathrm{km} \mathrm{s}^{-1}\right]}\end{array}$ & Ion & $\overline{\log N\left(\sigma_{\log N}\right)}$ & Comp. & $z_{\text {abs }}$ & $\begin{array}{c}v_{\mathrm{rel}}^{*} \\
{\left[\mathrm{~km} \mathrm{~s}^{-1}\right]}\end{array}$ & $\begin{array}{c}b\left(\sigma_{b}\right) \\
{\left[\mathrm{km} \mathrm{s}^{-1}\right]}\end{array}$ & Ion & $\overline{\log N\left(\sigma_{\log N}\right)}$ \\
\hline \multicolumn{12}{|c|}{ Low-ion transitions } \\
\hline \multirow[t]{5}{*}{1} & 2.06579 & -99 & $2.2(0.1)$ & $\mathrm{Fe}$ II & $12.98(0.02)$ & 7 & 2.06680 & 0 & $7.2(0.1)$ & Fe II & $13.59(0.01)$ \\
\hline & & & & Si II & $12.94(0.05)$ & & & & & Si II & $13.87(0.02)$ \\
\hline & & & & OI & $13.13(0.08)$ & & & & & O I & $>15.73$ \\
\hline & & & & $\mathrm{Al}$ II & $11.90(0.03)$ & & & & & $\mathrm{Al}$ II & $12.60(0.03)$ \\
\hline & & & & Ni II & $<12.35$ & & & & & S II & $13.53(0.05)$ \\
\hline \multirow[t]{6}{*}{2} & 2.06598 & -80 & $16.9(0.3)$ & Fe II & $13.14(0.01)$ & & & & & Ni II & $<12.66$ \\
\hline & & & & Si II & $13.75(0.02)$ & & & & & $\mathrm{N}_{\mathrm{I}}$ & $13.10(0.03)$ \\
\hline & & & & O I & $13.78(0.04)$ & & & & & P II & $11.90(0.15)$ \\
\hline & & & & Al II & $12.74(0.01)$ & & & & & Mg II & $14.97(0.04)$ \\
\hline & & & & Ni II & $<12.86$ & & & & & ArI & $<12.21$ \\
\hline & & & & $\mathrm{N}_{\mathrm{I}}$ & $12.71(0.07)$ & & & & & Cr II & $<11.92$ \\
\hline \multirow[t]{4}{*}{3} & 2.06603 & -75 & $4.9(0.2)$ & Fe II & $12.94(0.01)$ & 8 & 2.06698 & +18 & $8.6(0.3)$ & Fe II & $13.07(0.02)$ \\
\hline & & & & Si II & $13.51(0.03)$ & & & & & Si II & $13.51(0.01)$ \\
\hline & & & & O I & $13.37(0.08)$ & & & & & $\mathrm{O}_{\mathrm{I}}$ & $14.11(0.04)$ \\
\hline & & & & $\mathrm{Al}$ II & $12.48(0.02)$ & & & & & $\mathrm{Al}$ II & $12.47(0.01)$ \\
\hline \multirow[t]{11}{*}{4} & 2.06643 & -36 & $8.6(0.2)$ & Fe II & $13.63(0.01)$ & & & & & S II & $13.32(0.07)$ \\
\hline & & & & Si II & $13.95(0.02)$ & & & & & Ni II & $<12.17$ \\
\hline & & & & O I & $>14.63$ & & & & & $\mathrm{~N}_{\mathrm{I}}$ & $12.51(0.07)$ \\
\hline & & & & Al II & $12.70(0.08)$ & & & & & P II & $11.85(0.18)$ \\
\hline & & & & S II & $13.58(0.05)$ & & & & & Mg II & $14.77(0.06)$ \\
\hline & & & & Ni II & $<12.68$ & 9 & 2.06716 & +35 & $6.6(0.2)$ & Fe II & $12.98(0.01)$ \\
\hline & & & & N I & $12.90(0.04)$ & & & & & Si II & $13.30(0.02)$ \\
\hline & & & & P II & $12.35(0.10)$ & & & & & O I & $14.52(0.03)$ \\
\hline & & & & Mg II & $14.83(0.07)$ & & & & & $\mathrm{Al}$ II & $12.13(0.02)$ \\
\hline & & & & ArI & $<12.15$ & & & & & S II & $13.19(0.08)$ \\
\hline & & & & Cr II & $<12.01$ & & & & & Ni II & $<12.52$ \\
\hline \multirow[t]{11}{*}{5} & 2.06657 & -22 & $5.2(0.4)$ & Fe II & $13.47(0.03)$ & & & & & $\mathrm{N}_{\mathrm{I}}$ & $12.61(0.07)$ \\
\hline & & & & Si II & $13.75(0.03)$ & & & & & Mg II & $14.66(0.08)$ \\
\hline & & & & O I & $>14.60$ & 10 & 2.06758 & +76 & $3.2(0.3)$ & $\mathrm{Fe}$ II & $12.33(0.02)$ \\
\hline & & & & $\mathrm{Al}$ II & $12.36(0.03)$ & & & & & Si II & $12.63(0.08)$ \\
\hline & & & & S II & $13.37(0.06)$ & & & & & O I & $13.83(0.04)$ \\
\hline & & & & Ni II & $<12.53$ & & & & & $\mathrm{Al}$ II & $11.54(0.02)$ \\
\hline & & & & $\mathrm{NI}_{\mathrm{I}}$ & $12.90(0.04)$ & 11 & 2.06771 & +89 & $7.3(0.2)$ & Fe II & $12.75(0.01)$ \\
\hline & & & & P II & $11.76(0.19)$ & & & & & Si II & $13.17(0.03)$ \\
\hline & & & & Mg II & $14.90(0.04)$ & & & & & O I & $14.53(0.03)$ \\
\hline & & & & ArI & $<11.96$ & & & & & $\mathrm{Al}$ II & $11.88(0.01)$ \\
\hline & & & & Cr II & $<11.91$ & 12 & 2.06797 & +114 & $11.7(2.5)$ & Fe II & $12.17(0.08)$ \\
\hline \multirow[t]{12}{*}{6} & 2.06666 & -14 & $2.3(0.1)$ & $\mathrm{Fe}$ II & $13.37(0.02)$ & & & & & Si II & $12.60(0.02)$ \\
\hline & & & & Si II & $13.90(0.10)$ & & & & & O I & $13.61(0.03)$ \\
\hline & & & & OI & $>13.85$ & & & & & $\mathrm{Al}$ II & $11.45(0.03)$ \\
\hline & & & & Al II & $13.70(0.11)$ & 13 & 2.06811 & +128 & $3.1(0.8)$ & Fe II & $12.00(0.09)$ \\
\hline & & & & S II & $13.30(0.06)$ & & & & & Si II & $12.36(0.03)$ \\
\hline & & & & Ni II & $<12.63$ & & & & & O I & $13.59(0.04)$ \\
\hline & & & & $\mathrm{N}_{\mathrm{I}}$ & $12.51(0.06)$ & & & & & Al II & $10.74(0.10)$ \\
\hline & & & & P II & $12.15(0.11)$ & & & & & & \\
\hline & & & & Mg II & $14.69(0.07)$ & & & & & & \\
\hline & & & & ArI & $<11.88$ & & & & & & \\
\hline & & & & Cr II & $<11.71$ & & & & & & \\
\hline & & & & $\mathrm{C} \mathrm{II}^{*}$ & $12.57(0.11)$ & & & & & & \\
\hline \multicolumn{12}{|c|}{ Intermediate-ion transitions } \\
\hline \multirow[t]{3}{*}{1} & 2.06581 & -97 & $6.6(1.0)$ & $\mathrm{Al}$ III & $12.00(0.04)$ & 3 & 2.06603 & -75 & $8.0(0.3)$ & Al III & $12.65(0.01)$ \\
\hline & & & & Fe III & $13.20(0.06)$ & & & & & Fe III & $13.86(0.02)$ \\
\hline & & & & $\mathrm{N}$ II & $13.54(0.02)$ & & & & & $\mathrm{N}$ II & $13.99(0.01)$ \\
\hline \multirow[t]{3}{*}{2} & 2.06591 & -87 & $2.0(1.0)$ & Al III & $11.87(0.05)$ & 4 & 2.06640 & -39 & $13.0(0.9)$ & Al III & $12.42(0.03)$ \\
\hline & & & & Fe III & $12.91(0.12)$ & & & & & Fe III & $13.56(0.04)$ \\
\hline & & & & $\mathrm{N}$ II & $13.20(0.04)$ & & & & & $\mathrm{N}$ II & $13.78(0.01)$ \\
\hline
\end{tabular}


M. Dessauges-Zavadsky et al.: Data analysis and chemical variation studies, Online Material p 3

Table 2. continued.

\begin{tabular}{|c|c|c|c|c|c|c|c|c|c|c|c|}
\hline Comp. & $z_{\text {abs }}$ & $\begin{array}{c}v_{\text {rel }}^{*} \\
{\left[\mathrm{~km} \mathrm{~s}^{-1}\right]}\end{array}$ & $\begin{array}{c}b\left(\sigma_{b}\right) \\
{\left[\mathrm{km} \mathrm{s}^{-1}\right]}\end{array}$ & Ion & $\log N\left(\sigma_{\log N}\right)$ & Comp. & $z_{\mathrm{abs}}$ & $\begin{array}{c}v_{\mathrm{rel}}^{*} \\
{\left[\mathrm{~km} \mathrm{~s}^{-1}\right]}\end{array}$ & $\begin{array}{c}b\left(\sigma_{b}\right) \\
{\left[\mathrm{km} \mathrm{s}^{-1}\right]}\end{array}$ & Ion & $\log N\left(\sigma_{\log N}\right)$ \\
\hline 5 & 2.06666 & -14 & $9.8(0.9)$ & $\begin{array}{l}\mathrm{Al} \text { III } \\
\text { Fe III } \\
\mathrm{N} \text { II }\end{array}$ & $\begin{array}{l}12.35(0.04) \\
13.32(0.06) \\
13.86(0.01)\end{array}$ & 6 & 2.06700 & +19 & $16.2(1.7)$ & $\begin{array}{l}\mathrm{Al} \text { III } \\
\mathrm{Fe} \text { III } \\
\mathrm{N} \text { II }\end{array}$ & $\begin{array}{l}12.31(0.04) \\
13.75(0.04) \\
13.79(0.02)\end{array}$ \\
\hline
\end{tabular}

* Velocity relative to $z=2.06680$.

Note. In this and all the following tables describing the component structure of the DLAs studied, the encompassed components correspond to components which, according to our criterion, cannot be considered as individual ISM clouds on the QSO line of sight (see Sect. 5 for the definition of our criterion).

Table 3. Component structure of the $z_{\mathrm{abs}}=2.375$ DLA system toward Q $0841+129$.

\begin{tabular}{|c|c|c|c|c|c|c|c|c|c|c|c|}
\hline Comp. & $z_{\mathrm{abs}}$ & $\begin{array}{c}v_{\text {rel }}^{*} \\
{\left[\mathrm{~km} \mathrm{~s}^{-1}\right]}\end{array}$ & $\begin{array}{c}b\left(\sigma_{b}\right) \\
{\left[\mathrm{km} \mathrm{s}^{-1}\right]}\end{array}$ & Ion & $\log N\left(\sigma_{\log N}\right)$ & Comp. & $z_{\text {abs }}$ & $\begin{array}{c}v_{\text {rel }}^{*} \\
{\left[\mathrm{~km} \mathrm{~s}^{-1}\right]}\end{array}$ & $\begin{array}{c}b\left(\sigma_{b}\right) \\
{\left[\mathrm{km} \mathrm{s}^{-1}\right]}\end{array}$ & Ion & $\log N\left(\sigma_{\log N}\right)$ \\
\hline \multicolumn{12}{|c|}{ Low-ion transitions } \\
\hline \multirow[t]{4}{*}{1} & \multirow[t]{4}{*}{2.37407} & \multirow[t]{4}{*}{-40} & \multirow[t]{4}{*}{$2.0(1.0)$} & Fe II & $11.86(0.05)$ & \multirow[t]{15}{*}{4} & \multirow[t]{15}{*}{2.37452} & \multirow[t]{15}{*}{0} & \multirow[t]{15}{*}{$8.8(0.1)$} & Fe II & $14.69(0.01)$ \\
\hline & & & & Si II & $12.67(0.06)$ & & & & & Si II & $15.16(0.03)$ \\
\hline & & & & $\mathrm{Al}$ II & $11.37(0.08)$ & & & & & $\mathrm{Al}$ II & $>13.67$ \\
\hline & & & & O I & $13.10(0.05)$ & & & & & O I & $>15.76$ \\
\hline \multirow[t]{4}{*}{2} & \multirow[t]{4}{*}{2.37421} & \multirow[t]{4}{*}{-28} & \multirow[t]{4}{*}{$5.7(0.8)$} & Fe II & $12.39(0.05)$ & & & & & S II & $14.65(0.04)$ \\
\hline & & & & Si II & $13.15(0.04)$ & & & & & N I & $14.57(0.01)$ \\
\hline & & & & $\mathrm{Al}$ II & $11.81(0.05)$ & & & & & Mn II & $12.42(0.01)$ \\
\hline & & & & OI & $13.85(0.05)$ & & & & & Cr II & $13.05(0.01)$ \\
\hline \multirow[t]{15}{*}{3} & \multirow[t]{15}{*}{2.37435} & \multirow[t]{15}{*}{-15} & \multirow[t]{15}{*}{$6.6(0.4)$} & Fe II & $13.81(0.03)$ & & & & & Zn II & $12.10(0.02)$ \\
\hline & & & & Si II & $14.04(0.15)$ & & & & & Ni II & $13.45(0.03)$ \\
\hline & & & & $\mathrm{Al}$ II & $>12.54$ & & & & & Mg II & $15.13(0.10)$ \\
\hline & & & & O I & $>15.55$ & & & & & P II & $12.82(0.06)$ \\
\hline & & & & S II & $13.63(0.15)$ & & & & & Ar I & $13.53(0.08)$ \\
\hline & & & & N I & $13.49(0.02)$ & & & & & $\mathrm{C} \mathrm{II}^{*}$ & $12.96(0.08)$ \\
\hline & & & & Mn II & $11.70(0.07)$ & & & & & C I & $<12.89$ \\
\hline & & & & Cr II & $11.63(0.12)$ & \multirow[t]{4}{*}{5} & \multirow[t]{4}{*}{2.37479} & \multirow[t]{4}{*}{+24} & \multirow[t]{4}{*}{$4.2(0.1)$} & $\mathrm{Fe}$ II & $13.27(0.01)$ \\
\hline & & & & \multirow[t]{7}{*}{ Ni II } & \multirow[t]{7}{*}{$12.74(0.13)$} & & & & & Si II & $13.56(0.01)$ \\
\hline & & & & & & & & & & $\mathrm{Al}$ II & $11.98(0.03)$ \\
\hline & & & & & & & & & & O I & $>15.27$ \\
\hline & & & & & & 6 & 2.37493 & +36 & $3.0(1.0)$ & $\mathrm{Fe}$ II & $12.22(0.08)$ \\
\hline & & & & & & & & & & Si II & $12.43(0.07)$ \\
\hline & & & & & & & & & & $\mathrm{Al}$ II & $11.31(0.08)$ \\
\hline & & & & & & & & & & O I & $13.47(0.04)$ \\
\hline \multicolumn{12}{|c|}{ Intermediate-ion transitions } \\
\hline \multirow[t]{3}{*}{1} & \multirow[t]{3}{*}{2.37435} & \multirow[t]{3}{*}{-15} & \multirow[t]{3}{*}{$6.6(0.0)$} & $\mathrm{Al}$ III & $11.82(0.07)$ & \multirow[t]{3}{*}{2} & 2.37454 & +2 & $11.0(1.1)$ & $\mathrm{Al}$ III & $12.40(0.04)$ \\
\hline & & & & Fe III & $13.00(0.12)$ & & & & & Fe III & $13.75(0.03)$ \\
\hline & & & & S III & $<13.55$ & & & & & S III & $<14.04$ \\
\hline
\end{tabular}

\footnotetext{
* Velocity relative to $z=2.37452$.
} 
M. Dessauges-Zavadsky et al.: Data analysis and chemical variation studies, Online Material p 4

Table 4. Component structure of the $z_{\text {abs }}=2.476$ DLA system toward Q0841+129.

\begin{tabular}{|c|c|c|c|c|c|}
\hline Comp. & $z_{\mathrm{abs}}$ & $\begin{array}{c}v_{\mathrm{rel}}^{*} \\
{\left[\mathrm{~km} \mathrm{~s}^{-1}\right]}\end{array}$ & $\begin{array}{c}b\left(\sigma_{b}\right) \\
{\left[\mathrm{km} \mathrm{s}^{-1}\right]}\end{array}$ & Ion & $\log N\left(\sigma_{\log N}\right)$ \\
\hline \multicolumn{6}{|c|}{ Low- and intermediate-ion transitions } \\
\hline \multirow[t]{5}{*}{1} & 2.47597 & -21 & $15.0(2.0)$ & Fe II & $12.43(0.12)$ \\
\hline & & & & Si II & $12.83(0.12)$ \\
\hline & & & & O I & $13.74(0.20)$ \\
\hline & & & & $\mathrm{Al}$ II & $12.22(0.03)$ \\
\hline & & & & C II & $>13.88$ \\
\hline \multirow[t]{19}{*}{2} & 2.47621 & 0 & $10.2(0.1)$ & Fe II & $14.48(0.03)$ \\
\hline & & & & Si II & $14.96(0.02)$ \\
\hline & & & & $\mathrm{N}_{\mathrm{I}}$ & $13.91(0.08)$ \\
\hline & & & & S II & $14.45(0.10)$ \\
\hline & & & & OI & $16.11(0.09)$ \\
\hline & & & & Ar I & $13.09(0.08)$ \\
\hline & & & & $\mathrm{Al}$ II & $>13.26$ \\
\hline & & & & Cr II & $12.84(0.06)$ \\
\hline & & & & $\mathrm{Zn}$ II & $11.69(0.10)$ \\
\hline & & & & Mn II & $12.30(0.15)$ \\
\hline & & & & P II & $12.50(0.07)$ \\
\hline & & & & Ni II & $13.15(0.07)$ \\
\hline & & & & Mg II & $14.99(0.15)$ \\
\hline & & & & $\mathrm{C}$ II & $>15.52$ \\
\hline & & & & $\mathrm{C}_{\mathrm{II}^{*}}$ & $<13.08$ \\
\hline & & & & $\mathrm{Al}$ III & $12.54(0.02)$ \\
\hline & & & & Fe III & $<13.55$ \\
\hline & & & & N II & $<13.54$ \\
\hline & & & & S III & $<13.72$ \\
\hline \multirow[t]{12}{*}{3} & 2.47642 & +18 & $2.3(0.3)$ & Fe II & $13.15(0.05)$ \\
\hline & & & & Si II & $13.83(0.16)$ \\
\hline & & & & $\mathrm{NI}_{\mathrm{I}}$ & $12.80(0.10)$ \\
\hline & & & & S II & $13.31(0.08)$ \\
\hline & & & & O I & $15.11(0.19)$ \\
\hline & & & & Ar I & $11.79(0.15)$ \\
\hline & & & & $\mathrm{Al}$ II & $>12.54$ \\
\hline & & & & Cr II & $11.91(0.09)$ \\
\hline & & & & Mn II & $11.17(0.20)$ \\
\hline & & & & P II & $11.70(0.20)$ \\
\hline & & & & Ni II & $12.15(0.20)$ \\
\hline & & & & C II & $>15.74$ \\
\hline
\end{tabular}

$*$ Velocity relative to $z=2.47621$.

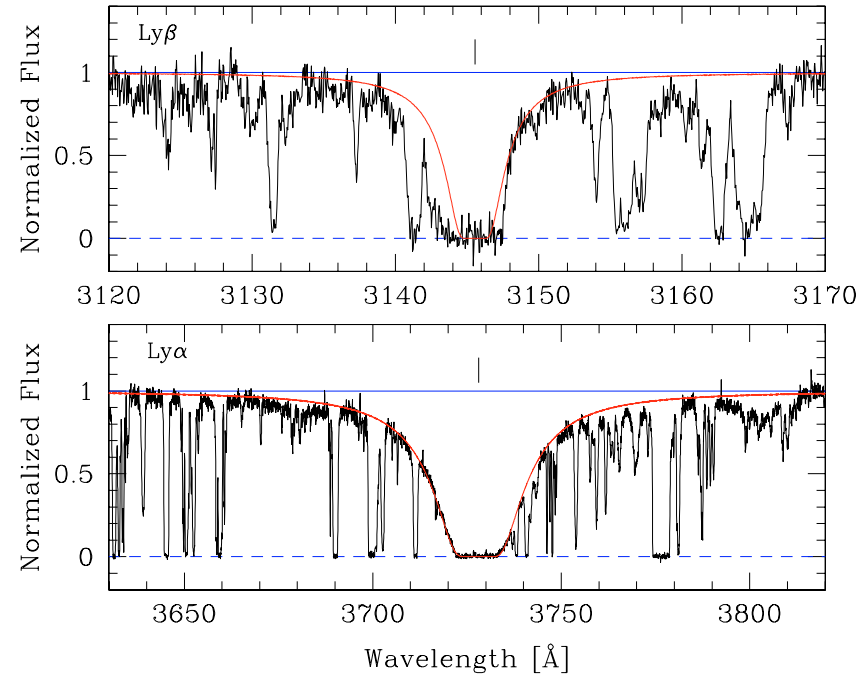

Fig. 1. Normalized UVES spectrum of Q0450-13 showing the DLA $\operatorname{Ly} \alpha$ and $\operatorname{Ly} \beta$ line profiles with the Voigt profile fits. The vertical bar corresponds to the wavelength centroid of the component used for the best fit, $z=2.06666$. The measured H I column density is $\log N(\mathrm{HI})=20.53 \pm 0.08$. 
M. Dessauges-Zavadsky et al.: Data analysis and chemical variation studies, Online Material p 5

Table 5. Component structure of the $z_{\text {abs }}=1.944$ DLA system toward Q1157+014.

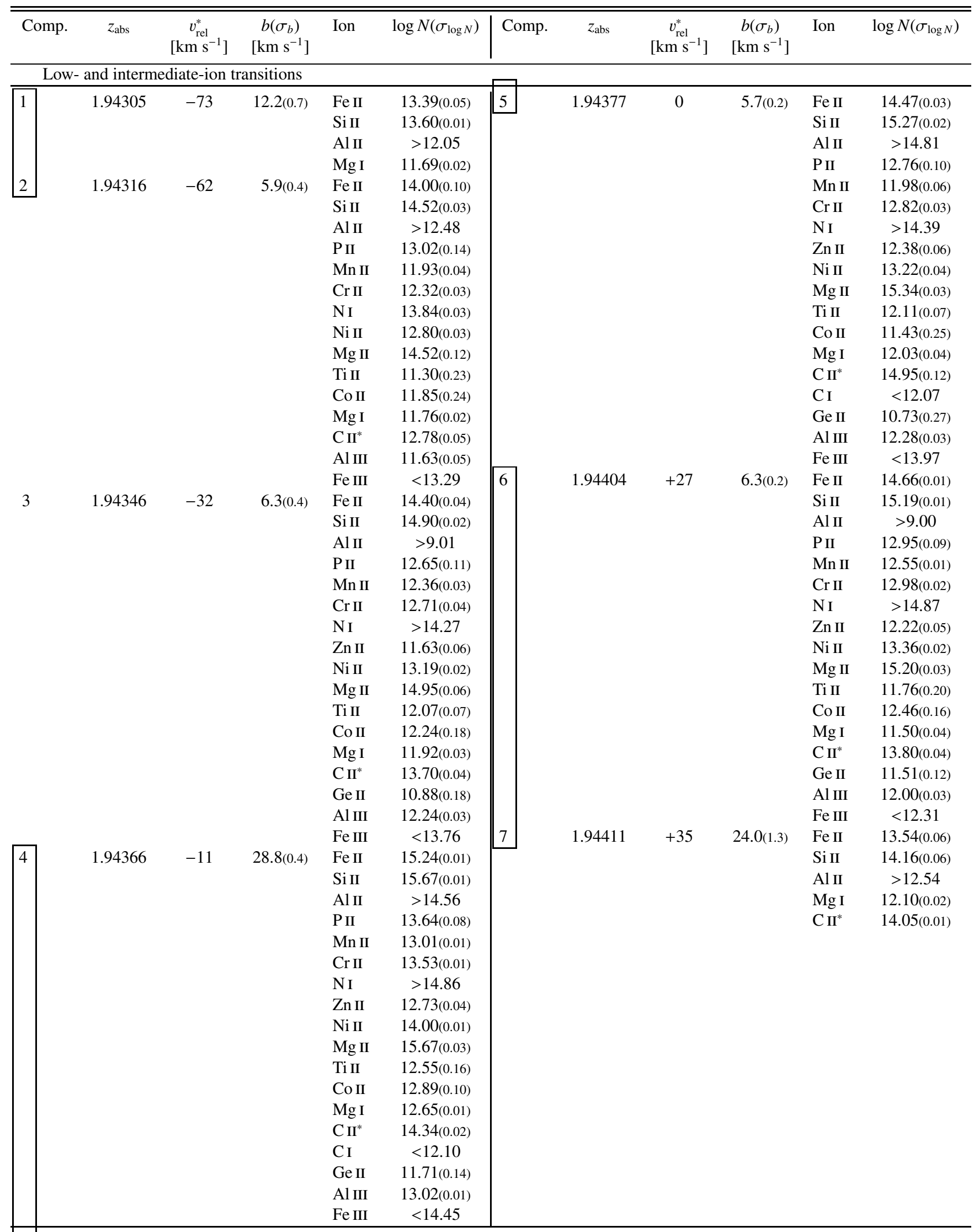

* Nelocity relative to $z=1.94377$. 
M. Dessauges-Zavadsky et al.: Data analysis and chemical variation studies, Online Material p 6
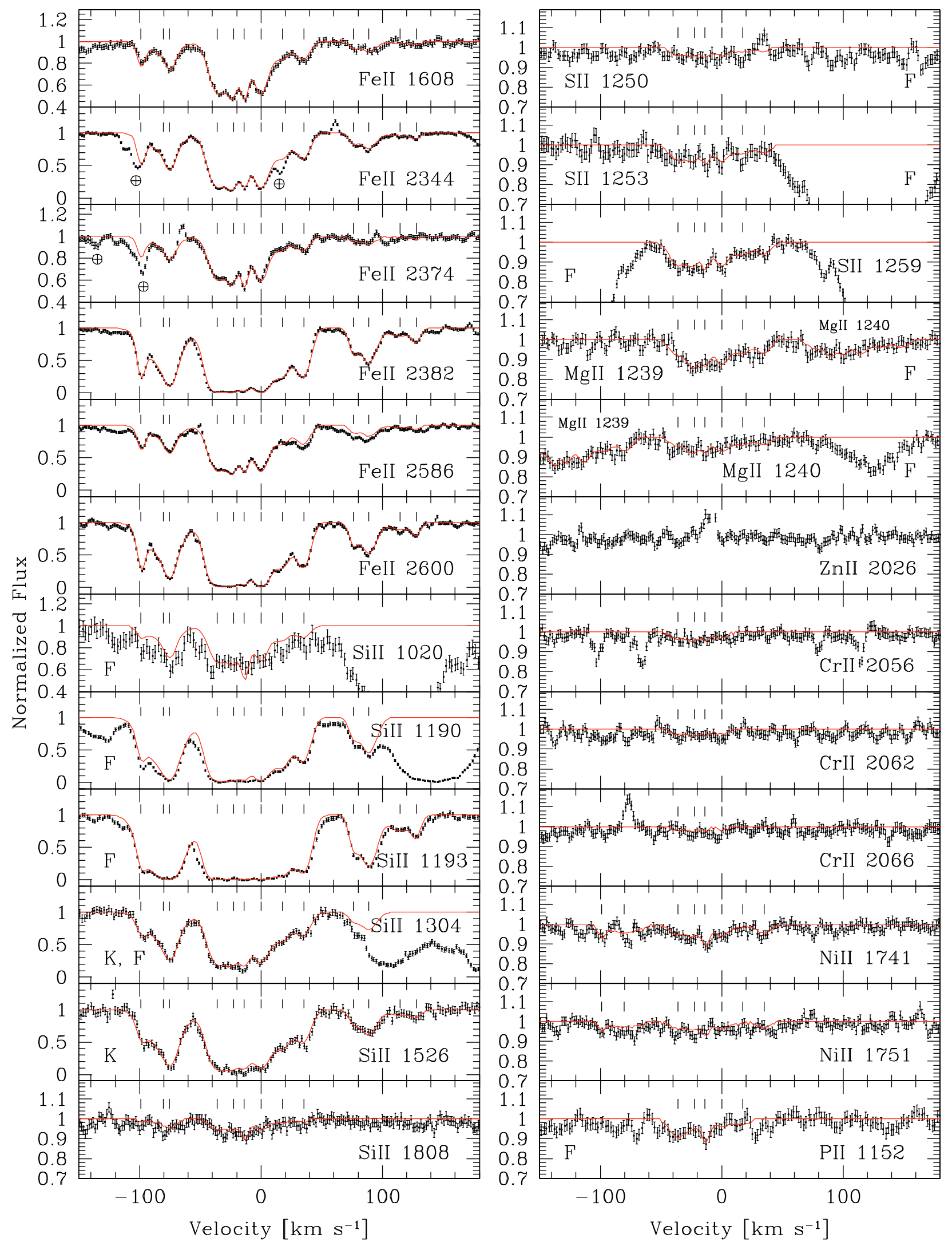

Fig. 2. Velocity plots of the metal line transitions (normalized intensities shown by dots with $1 \sigma$ error bars) for the DLA toward Q0450-13. The zero velocity is fixed at $z=2.06680$. For this and all the following figures with velocity plots, the vertical bars mark the positions of the fitted velocity components, and the symbol $\oplus$ corresponds to telluric lines. The letter K refers to Keck spectra and the letter F to the Ly $\alpha$ forest. 
M. Dessauges-Zavadsky et al.: Data analysis and chemical variation studies, Online Material p 7
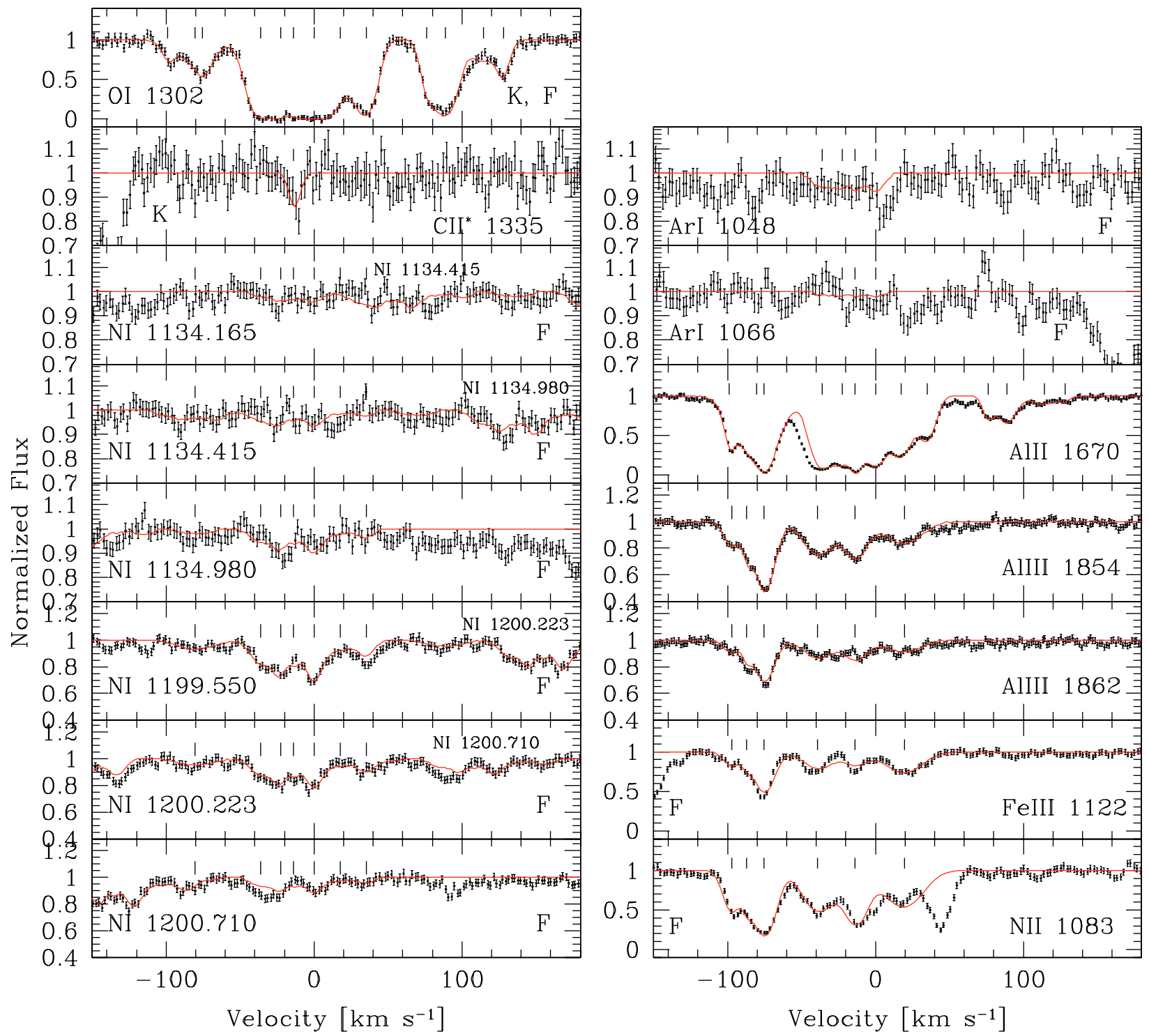

Fig. 2. continued. 
M. Dessauges-Zavadsky et al.: Data analysis and chemical variation studies, Online Material $p 8$

Table 6. Component structure of the $z_{\text {abs }}=1.892$ DLA system toward Q1210+17.

\begin{tabular}{|c|c|c|c|c|c|}
\hline Comp. & $z_{\mathrm{abs}}$ & $\begin{array}{c}v_{\text {rel }}^{*} \\
{\left[\mathrm{~km} \mathrm{~s}^{-1}\right]}\end{array}$ & $\begin{array}{c}b\left(\sigma_{b}\right) \\
{\left[\mathrm{km} \mathrm{s}^{-1}\right]}\end{array}$ & Ion & $\log N\left(\sigma_{\log N}\right)$ \\
\hline \multicolumn{6}{|c|}{ Low- and intermediate-ion transitions } \\
\hline \multirow[t]{5}{*}{1} & 1.89126 & -53 & $3.0(0.4)$ & Fe II & $12.85(0.02)$ \\
\hline & & & & Si II & $13.10(0.03)$ \\
\hline & & & & Al II & $11.93(0.04)$ \\
\hline & & & & P II & $<12.77$ \\
\hline & & & & $\mathrm{Mg}$ II & $>13.74$ \\
\hline \multirow[t]{5}{*}{2} & 1.89140 & -38 & $5.4(0.2)$ & Fe II & $13.55(0.01)$ \\
\hline & & & & Si II & $13.75(0.02)$ \\
\hline & & & & $\mathrm{Al}$ II & $12.41(0.03)$ \\
\hline & & & & P II & $<13.09$ \\
\hline & & & & Mg II & $>13.58$ \\
\hline \multirow[t]{12}{*}{3} & 1.89158 & -20 & 7.7(0.8) & $\mathrm{Fe}$ II & $14.19(0.02)$ \\
\hline & & & & Si II & $14.43(0.06)$ \\
\hline & & & & $\mathrm{Al}$ II & $>13.20$ \\
\hline & & & & S II & $14.09(0.02)$ \\
\hline & & & & Mn II & $11.91(0.02)$ \\
\hline & & & & Cr II & $12.55(0.05)$ \\
\hline & & & & Ni & $13.83(0.06)$ \\
\hline & & & & Zn II & $11.45(0.17)$ \\
\hline & & & & Ni II & $12.86(0.08)$ \\
\hline & & & & P II & $<13.27$ \\
\hline & & & & Mg II & $>14.22$ \\
\hline & & & & Al III & $12.36(0.03)$ \\
\hline \multirow[t]{13}{*}{4} & 1.89177 & 0 & $5.8(0.1)$ & $\mathrm{Fe}$ II & $14.84(0.03)$ \\
\hline & & & & Si II & $15.19(0.02)$ \\
\hline & & & & $\mathrm{Al}$ II & $>14.93$ \\
\hline & & & & S II & $14.81(0.01)$ \\
\hline & & & & Mn II & $12.57(0.01)$ \\
\hline & & & & Cr II & $13.11(0.02)$ \\
\hline & & & & N I & $14.59(0.10)$ \\
\hline & & & & Zn II & $12.29(0.02)$ \\
\hline & & & & Ni II & $13.52(0.05)$ \\
\hline & & & & Ti II & $12.34(0.08)$ \\
\hline & & & & P II & $<13.72$ \\
\hline & & & & Mg II & $<15.51$ \\
\hline & & & & Al III & $12.77(0.02)$ \\
\hline \multirow[t]{11}{*}{5} & 1.89192 & +16 & $7.6(0.1)$ & $\mathrm{Fe}$ II & $14.13(0.01)$ \\
\hline & & & & Si II & $14.38(0.06)$ \\
\hline & & & & $\mathrm{Al}$ II & $>12.76$ \\
\hline & & & & S II & $14.13(0.05)$ \\
\hline & & & & Mn II & $11.88(0.02)$ \\
\hline & & & & Cr II & $12.45(0.07)$ \\
\hline & & & & N I & $13.78(0.10)$ \\
\hline & & & & Zn II & $11.44(0.17)$ \\
\hline & & & & Ni II & $12.82(0.08)$ \\
\hline & & & & Mg II & $>14.71$ \\
\hline & & & & Al III & $12.17(0.05)$ \\
\hline 6 & 1.89222 & +47 & $11.7(0.5)$ & Mg II & $12.34(0.04)$ \\
\hline \multirow[t]{4}{*}{7} & 1.89242 & +67 & $4.4(0.2)$ & Fe II & $12.57(0.01)$ \\
\hline & & & & Si II & $12.90(0.04)$ \\
\hline & & & & Al II & $11.26(0.10)$ \\
\hline & & & & Mg II & $12.89(0.01)$ \\
\hline
\end{tabular}

$*$ Velocity relative to $z=1.89177$

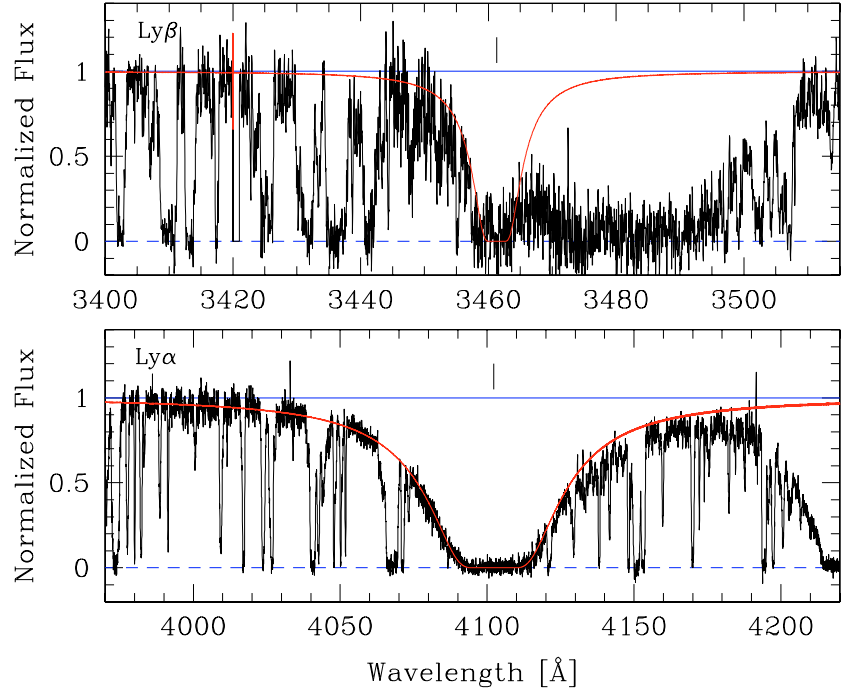

Fig. 3. Normalized UVES spectrum of Q0841+129 showing the $z_{\mathrm{abs}}=$ 2.375 DLA Ly $\alpha$ and $\operatorname{Ly} \beta$ line profiles with the Voigt profile fits. The vertical bar corresponds to the wavelength centroid of the component used for the best fit, $z=2.37452$. The measured $\mathrm{H}$ I column density is $\log N(\mathrm{HI})=20.99 \pm 0.08$. 
M. Dessauges-Zavadsky et al.: Data analysis and chemical variation studies, Online Material p 9

Table 7. Component structure of the $z_{\mathrm{abs}}=1.864$ DLA system toward Q2230+02.

\begin{tabular}{|c|c|c|c|c|c|c|c|c|c|c|c|}
\hline comp. & $z_{\mathrm{abs}}$ & $\begin{array}{c}v_{\text {rel }}^{*} \\
{\left[\mathrm{~km} \mathrm{~s}^{-1}\right]}\end{array}$ & $\begin{array}{c}b\left(\sigma_{b}\right) \\
{\left[\mathrm{km} \mathrm{s}^{-1}\right]}\end{array}$ & Ion & $\log N\left(\sigma_{\log N}\right)$ & Comp. & $z_{\mathrm{abs}}$ & $\begin{array}{c}v_{\mathrm{rel}}^{*} \\
{\left[\mathrm{~km} \mathrm{~s}^{-1}\right]}\end{array}$ & $\begin{array}{c}b\left(\sigma_{b}\right) \\
{\left[\mathrm{km} \mathrm{s}^{-1}\right]}\end{array}$ & Ion & $\overline{\log N\left(\sigma_{\log N}\right)}$ \\
\hline \multicolumn{12}{|c|}{ Low-ion transitions } \\
\hline \multirow[t]{3}{*}{1} & 1.86183 & -184 & $4.3(0.3)$ & Fe II & $12.94(0.04)$ & 12 & 1.86375 & +17 & $6.0(0.4)$ & $\mathrm{Fe}$ II & $14.40(0.04)$ \\
\hline & & & & Si II & $13.60(0.08)$ & & & & & Si II & $14.96(0.02)$ \\
\hline & & & & $\mathrm{Al}$ II & $12.16(0.06)$ & & & & & $S_{\text {II }}$ & $14.52(0.04)$ \\
\hline \multirow[t]{3}{*}{2} & 1.86191 & -176 & $10.0(0.5)$ & Fe II & $12.93(0.04)$ & & & & & Mn II & $12.35(0.02)$ \\
\hline & & & & $\mathrm{Si}$ II & $13.16(0.08)$ & & & & & Mg I & $12.11(0.01)$ \\
\hline & & & & $\mathrm{Al}$ II & $12.06(0.06)$ & & & & & $\mathrm{C} \mathrm{II}^{*}$ & $13.23(0.01)$ \\
\hline \multirow[t]{4}{*}{3} & 1.86214 & -152 & $5.5(0.3)$ & Fe II & $12.91(0.04)$ & & & & & $\mathrm{N} \mathrm{I}$ & $14.44(0.14)$ \\
\hline & & & & $\mathrm{Si}$ II & $13.52(0.06)$ & & & & & Cr II & $12.80(0.04)$ \\
\hline & & & & O I & $13.70(0.03)$ & & & & & Zn II & $12.09(0.04)$ \\
\hline & & & & $\mathrm{Al}$ II & $12.28(0.04)$ & & & & & Ni II & $13.33(0.02)$ \\
\hline \multirow[t]{4}{*}{4} & 1.86223 & -142 & $9.0(1.3)$ & $\mathrm{Fe}$ II & $12.52(0.10)$ & & & & & $\mathrm{P}_{\mathrm{II}}$ & $13.07(0.08)$ \\
\hline & & & & Si II & $12.84(0.17)$ & & & & & Ti II & $12.19(0.08)$ \\
\hline & & & & O I & $13.33(0.06)$ & 13 & 1.86399 & +42 & $14.5(0.7)$ & $\mathrm{Fe}$ II & $14.34(0.03)$ \\
\hline & & & & $\mathrm{Al}$ II & $11.57(0.17)$ & & & & & Si II & $14.81(0.03)$ \\
\hline \multirow[t]{3}{*}{5} & 1.86262 & -102 & $6.7(0.3)$ & Fe II & $13.52(0.01)$ & & & & & S II & $14.61(0.08)$ \\
\hline & & & & Si II & $13.91(0.02)$ & & & & & Mn II & $12.18(0.04)$ \\
\hline & & & & $\operatorname{Mg} I$ & $11.47(0.03)$ & & & & & Mg I & $12.22(0.01)$ \\
\hline \multirow[t]{3}{*}{6} & 1.86274 & -89 & $2.5(0.4)$ & $\mathrm{Fe}$ II & $13.23(0.04)$ & & & & & $\mathrm{C} \mathrm{II}^{*}$ & $13.18(0.02)$ \\
\hline & & & & Si II & $13.58(0.07)$ & & & & & N I & $14.12(0.03)$ \\
\hline & & & & Mg I & $10.93(0.08)$ & & & & & Cr II & $12.46(0.11)$ \\
\hline \multirow[t]{3}{*}{7} & 1.86284 & -79 & $7.4(0.4)$ & $\mathrm{Fe}$ II & $13.74(0.02)$ & & & & & Zn II & $11.88(0.09)$ \\
\hline & & & & Si II & $14.34(0.02)$ & & & & & Ni II & $13.30(0.03)$ \\
\hline & & & & $\mathrm{Mg}_{\mathrm{I}}$ & $11.54(0.03)$ & & & & & $\mathrm{P}_{\text {II }}$ & $13.08(0.08)$ \\
\hline \multirow[t]{6}{*}{8} & 1.86305 & -57 & $9.4(0.6)$ & $\mathrm{Fe}$ II & $13.91(0.03)$ & 14 & 1.86430 & +74 & $17.3(0.7)$ & $\mathrm{Fe}$ II & $14.70(0.03)$ \\
\hline & & & & Si II & $14.29(0.07)$ & & & & & Si II & $15.11(0.02)$ \\
\hline & & & & Mn II & $11.92(0.05)$ & & & & & S II & $14.80(0.06)$ \\
\hline & & & & Mg I & $11.42(0.03)$ & & & & & Mn II & $12.48(0.02)$ \\
\hline & & & & N I & $13.68(0.03)$ & & & & & Mg I & $12.30(0.10)$ \\
\hline & & & & Ni II & $12.90(0.17)$ & & & & & $\mathrm{C}$ II $^{*}$ & $13.22(0.02)$ \\
\hline \multirow[t]{12}{*}{9} & 1.86320 & -41 & $8.3(0.4)$ & $\mathrm{Fe}$ II & $14.58(0.02)$ & & & & & N I & $14.46(0.03)$ \\
\hline & & & & Si II & $15.00(0.02)$ & & & & & Cr II & $12.98(0.04)$ \\
\hline & & & & $S_{\text {II }}$ & $14.61(0.04)$ & & & & & Zn II & $12.30(0.04)$ \\
\hline & & & & Mn II & $12.45(0.02)$ & & & & & Ni II & $13.58(0.02)$ \\
\hline & & & & $\mathrm{Mg}_{\mathrm{I}}$ & $11.93(0.02)$ & & & & & $\mathrm{P}_{\text {II }}$ & $13.17(0.07)$ \\
\hline & & & & $\mathrm{C} \mathrm{II}^{*}$ & $<13.32$ & & & & & Ti II & $12.25(0.12)$ \\
\hline & & & & $\mathrm{NI}$ & $14.39(0.03)$ & 15 & 1.86445 & +90 & $5.1(1.5)$ & $\mathrm{Fe}$ II & $13.42(0.04)$ \\
\hline & & & & Cr II & $12.94(0.03)$ & & & & & Si II & $13.83(0.10)$ \\
\hline & & & & Zn II & $12.09(0.06)$ & & & & & $\mathrm{Al}$ II & $14.08(0.11)$ \\
\hline & & & & Ni II & $13.54(0.02)$ & & & & & Mg I & $11.31(0.04)$ \\
\hline & & & & P II & $13.01(0.09)$ & 16 & 1.86468 & +114 & $5.6(0.3)$ & $\mathrm{Fe}$ II & $13.11(0.04)$ \\
\hline & & & & Ti II & $12.17(0.08)$ & & & & & Si II & $13.61(0.13)$ \\
\hline \multirow[t]{6}{*}{10} & 1.86338 & -22 & $12.9(0.9)$ & Fe II & $13.81(0.03)$ & & & & & Al II & $12.38(0.04)$ \\
\hline & & & & Si II & $14.26(0.02)$ & 17 & 1.86478 & +125 & $10.2(0.4)$ & $\mathrm{Fe}$ II & $13.26(0.03)$ \\
\hline & & & & $S_{\text {II }}$ & $13.99(0.10)$ & & & & & $\mathrm{Si}$ II & $13.64(0.07)$ \\
\hline & & & & $\operatorname{Mg}_{\mathrm{I}}$ & $11.54(0.03)$ & & & & & $\mathrm{Al}$ II & $12.23(0.03)$ \\
\hline & & & & $\mathrm{C} \mathrm{II}^{*}$ & $13.15(0.02)$ & & & & & $\operatorname{Mg} I$ & $11.62(0.03)$ \\
\hline & & & & N I & $13.67(0.04)$ & 18 & 1.86502 & +150 & $6.9(0.2)$ & Fe II & $13.19(0.01)$ \\
\hline \multirow[t]{8}{*}{11} & 1.86359 & 0 & $6.5(0.2)$ & $\mathrm{Fe}$ II & $13.65(0.01)$ & & & & & Si II & $13.57(0.10)$ \\
\hline & & & & Si II & $14.11(0.09)$ & & & & & OI & $14.47(0.02)$ \\
\hline & & & & $S_{\text {II }}$ & $13.83(0.21)$ & & & & & Al II & $12.30(0.02)$ \\
\hline & & & & Mn II & $11.54(0.10)$ & & & & & Mg I & $11.58(0.03)$ \\
\hline & & & & Mg I & $11.68(0.02)$ & 19 & 1.86519 & +168 & $9.0(0.6)$ & Fe II & $12.53(0.02)$ \\
\hline & & & & $\mathrm{C} \mathrm{II}^{*}$ & $12.69(0.03)$ & & & & & $\mathrm{Si}$ II & $13.12(0.09)$ \\
\hline & & & & $\mathrm{NI}_{\mathrm{I}}$ & $13.21(0.06)$ & & & & & OI & $13.82(0.02)$ \\
\hline & & & & Ni II & $12.41(0.20)$ & & & & & $\mathrm{Al}$ II & $11.87(0.04)$ \\
\hline
\end{tabular}


M. Dessauges-Zavadsky et al.: Data analysis and chemical variation studies, Online Material p 10

Table 7. continued.

\begin{tabular}{|c|c|c|c|c|c|c|c|c|c|c|c|}
\hline No & $z_{\mathrm{abs}}$ & $\begin{array}{c}v_{\text {rel }}^{*} \\
\mathrm{~km} \mathrm{~s}^{-1}\end{array}$ & $\begin{array}{c}b\left(\sigma_{b}\right) \\
\mathrm{km} \mathrm{s}^{-1}\end{array}$ & Ion & $\log N\left(\sigma_{\log N}\right)$ & No & $z_{\mathrm{abs}}$ & $\begin{array}{c}v_{\mathrm{rel}}^{*} \\
\mathrm{~km} \mathrm{~s}^{-1}\end{array}$ & $\begin{array}{c}b\left(\sigma_{b}\right) \\
\mathrm{km} \mathrm{s}^{-1}\end{array}$ & Ion & $\log N\left(\sigma_{\log N}\right)$ \\
\hline 20 & 1.86548 & +198 & $14.6(0.8)$ & $\begin{array}{l}\text { Fe II } \\
\text { Si II } \\
\text { O I } \\
\text { Al II }\end{array}$ & $\begin{array}{l}12.73(0.02) \\
13.23(0.08) \\
14.07(0.01) \\
11.81(0.05) \\
\end{array}$ & 21 & 1.86576 & +227 & $6.0(1.7)$ & Fe II & $11.85(0.07)$ \\
\hline \multicolumn{12}{|c|}{ Intermediate-ion transitions } \\
\hline $\begin{array}{l}1 \\
2\end{array}$ & $\begin{array}{l}1.86262 \\
1.86274\end{array}$ & $\begin{array}{c}-102 \\
-89\end{array}$ & $\begin{array}{l}6.7(0.3) \\
2.5(0.4)\end{array}$ & $\begin{array}{l}\mathrm{Al} \text { III } \\
\mathrm{Al} \text { III }\end{array}$ & $\begin{array}{l}12.25(0.03) \\
12.28(0.04)\end{array}$ & 8 & 1.86375 & +17 & $6.0(0.4)$ & $\begin{array}{l}\mathrm{Al} \text { III } \\
\mathrm{Fe} \text { III }\end{array}$ & $\begin{array}{c}12.75(0.01) \\
<13.74\end{array}$ \\
\hline 3 & 1.86284 & $\begin{array}{r}-79 \\
57\end{array}$ & 7.4(0.4) & $\mathrm{Al}$ III & $12.53(0.02)$ & 9 & 1.86399 & +42 & $14.5(0.7)$ & $\begin{array}{l}\mathrm{Al} \text { III } \\
\mathrm{Fe} \text { II }\end{array}$ & $\begin{array}{c}12.71(0.02) \\
<13.72\end{array}$ \\
\hline $\begin{array}{l}4 \\
5\end{array}$ & $\begin{array}{l}1.86305 \\
1.86320\end{array}$ & $\begin{array}{l}-5 / \\
-41\end{array}$ & $\begin{array}{l}9.4(0.6) \\
8.3(0.4)\end{array}$ & $\begin{array}{l}\text { AlIII } \\
\text { Fe III }\end{array}$ & $\begin{array}{c}12.31(0.03) \\
12.91(0.01) \\
<13.90\end{array}$ & 10 & 1.86432 & +76 & $11.2(0.6)$ & $\begin{array}{l}\mathrm{Al} \text { III } \\
\mathrm{Fe} \text { III }\end{array}$ & $\begin{array}{c}<13.12 \\
12.91(0.02) \\
<13.92\end{array}$ \\
\hline 6 & 1.86338 & -22 & $12.9(0.9)$ & $\begin{array}{l}\mathrm{Al} \text { III } \\
\text { Fe III }\end{array}$ & $\begin{array}{c}12.18(0.04) \\
<13.73\end{array}$ & 11 & 1.86450 & +95 & $4.8(1.5)$ & $\begin{array}{l}\mathrm{Al} \text { III } \\
\mathrm{Fe} \text { III }\end{array}$ & $\begin{array}{c}11.99(0.09) \\
<13.05\end{array}$ \\
\hline 7 & 1.86359 & 0 & $6.5(0.2)$ & $\begin{array}{l}\mathrm{Al} \text { III } \\
\text { Fe III }\end{array}$ & $\begin{array}{c}11.82(0.07) \\
<13.46\end{array}$ & & & & & & \\
\hline
\end{tabular}

${ }^{*}$ Velocity relative to $z=1.86359$ 
M. Dessauges-Zavadsky et al.: Data analysis and chemical variation studies, Online Material p 11
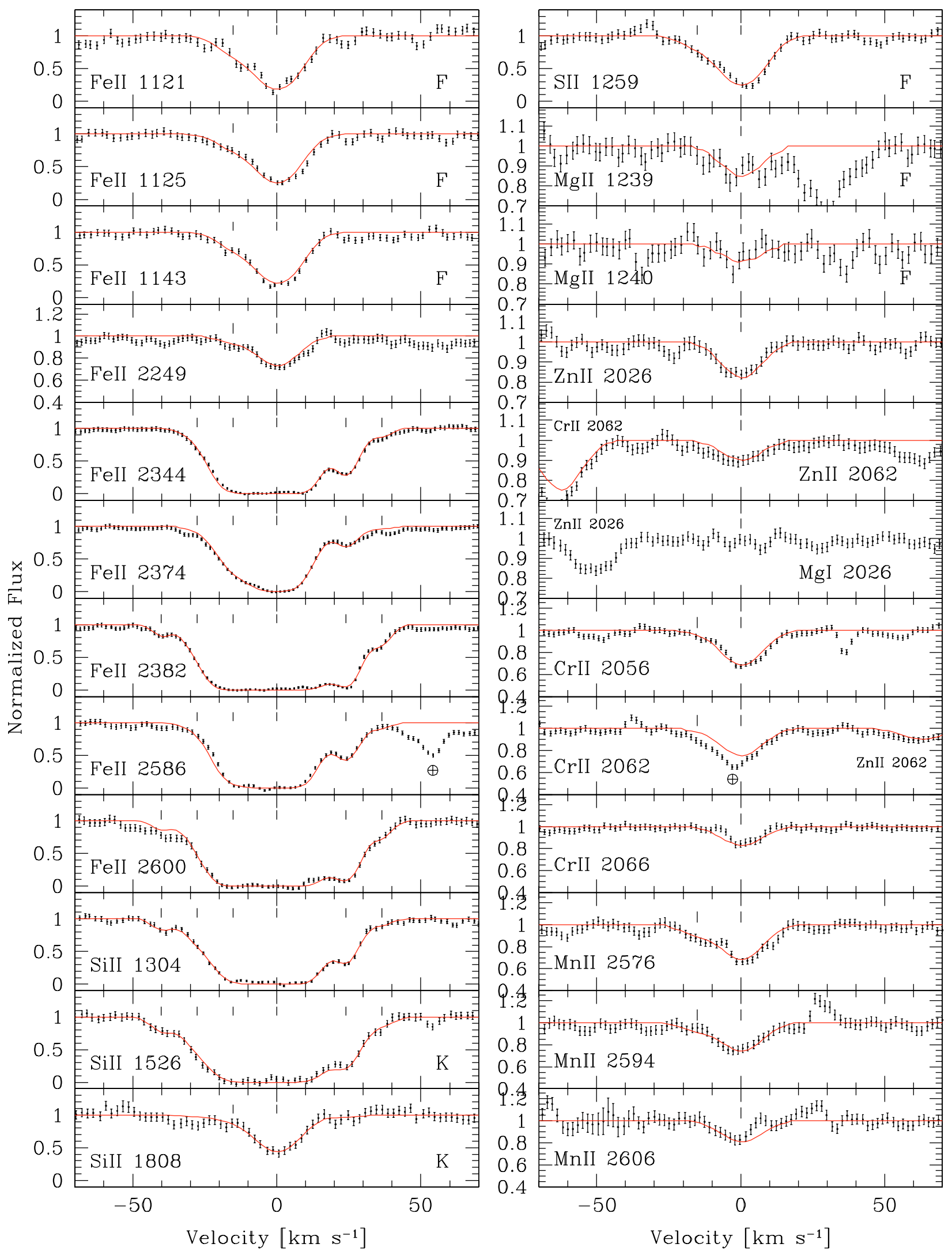

Fig. 4. Same as Fig. 2 for the first DLA toward Q0841+129 at $z_{\text {abs }}=2.375$. The zero velocity is fixed at $z=2.37452$. 
M. Dessauges-Zavadsky et al.: Data analysis and chemical variation studies, Online Material p 12
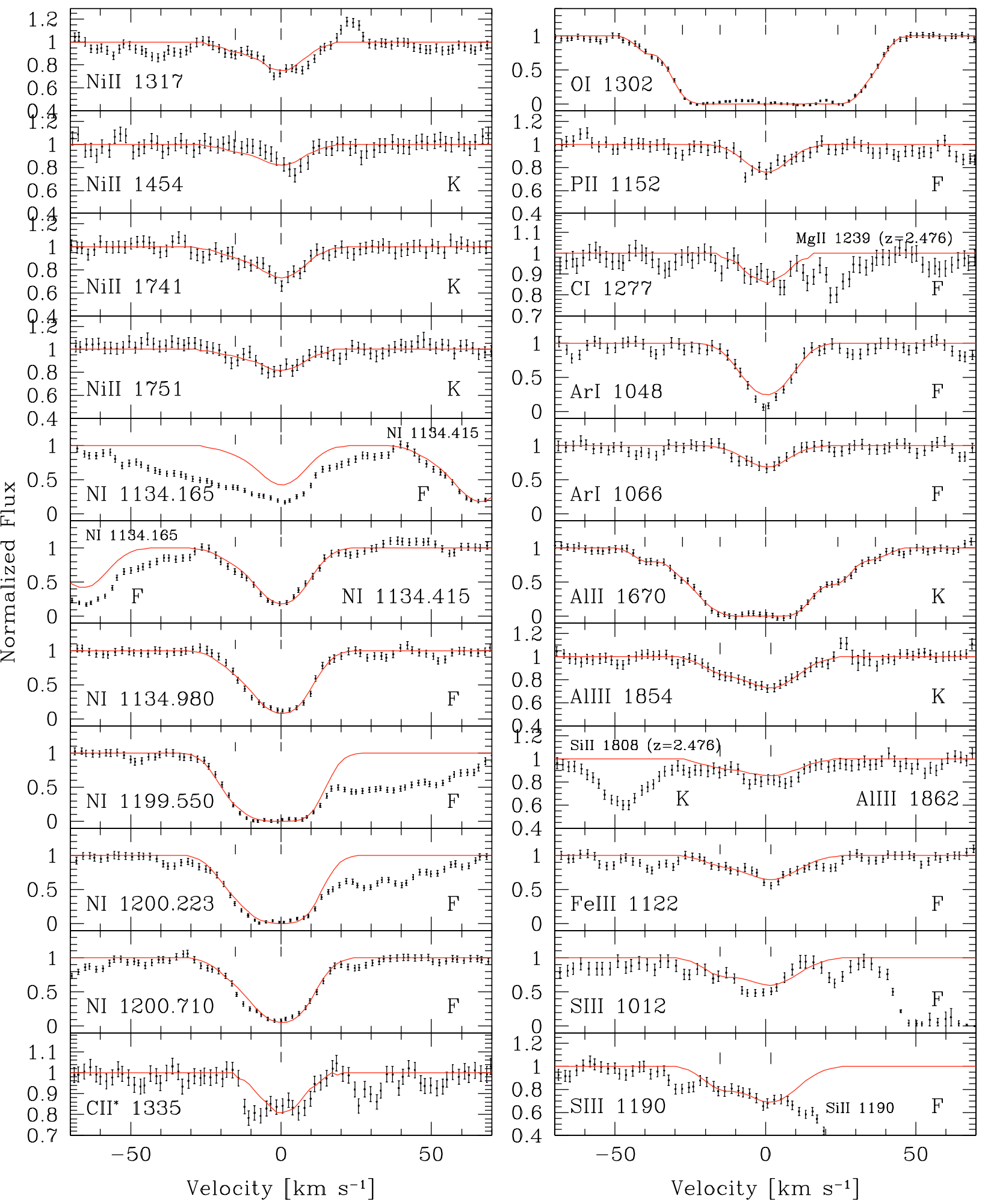

Fig. 4. continued. 
M. Dessauges-Zavadsky et al.: Data analysis and chemical variation studies, Online Material p 13

Table 8. Component structure of the $z_{\mathrm{abs}}=2.279$ DLA system toward Q2348-1444.

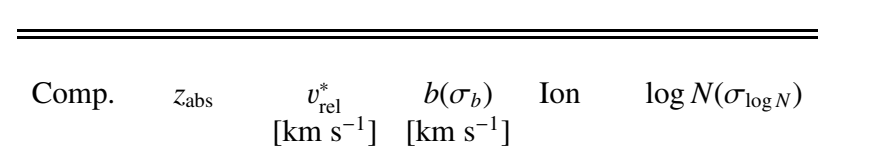

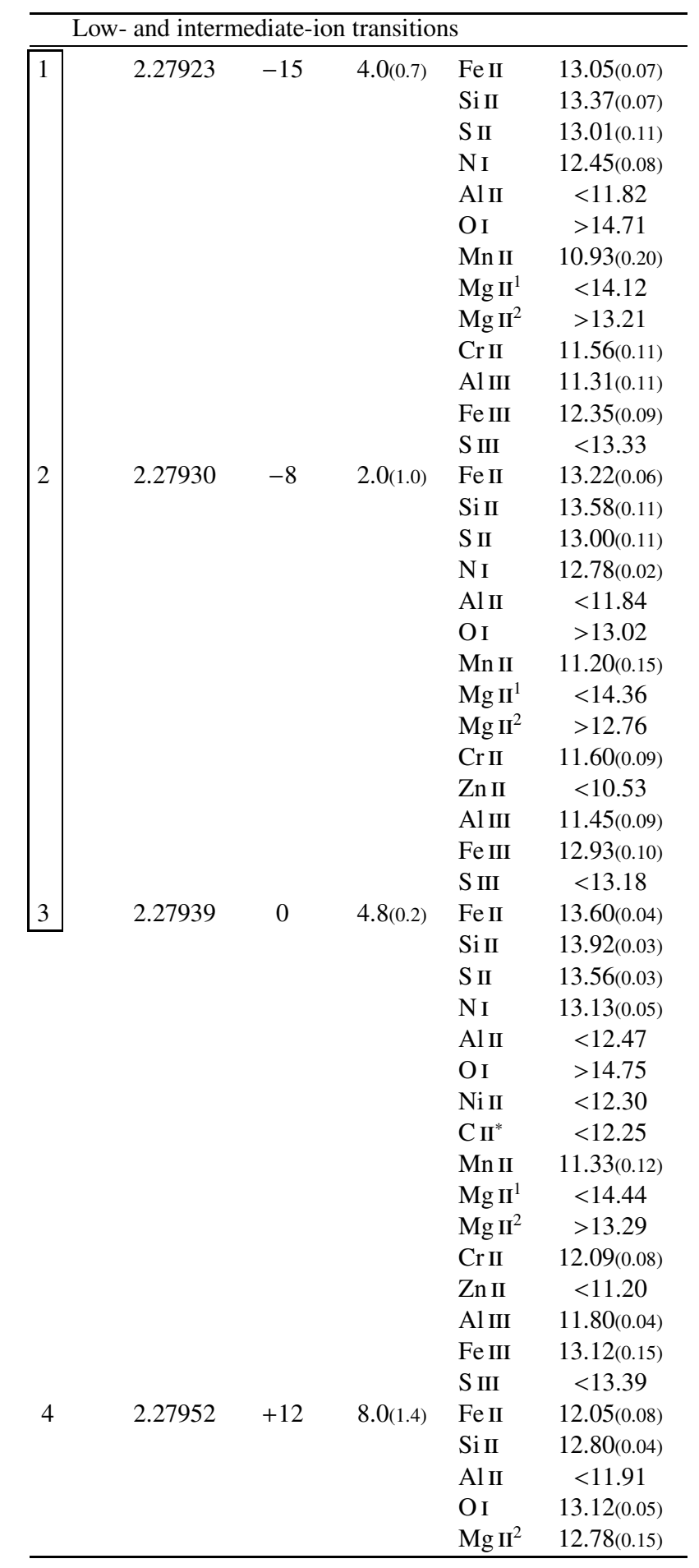

* Velocity relative to $z=2.27939$.

${ }^{1} \mathrm{Mg}$ II $\lambda 1239,1240$.

${ }^{2} \mathrm{Mg}$ II $\lambda 2793,2803$.

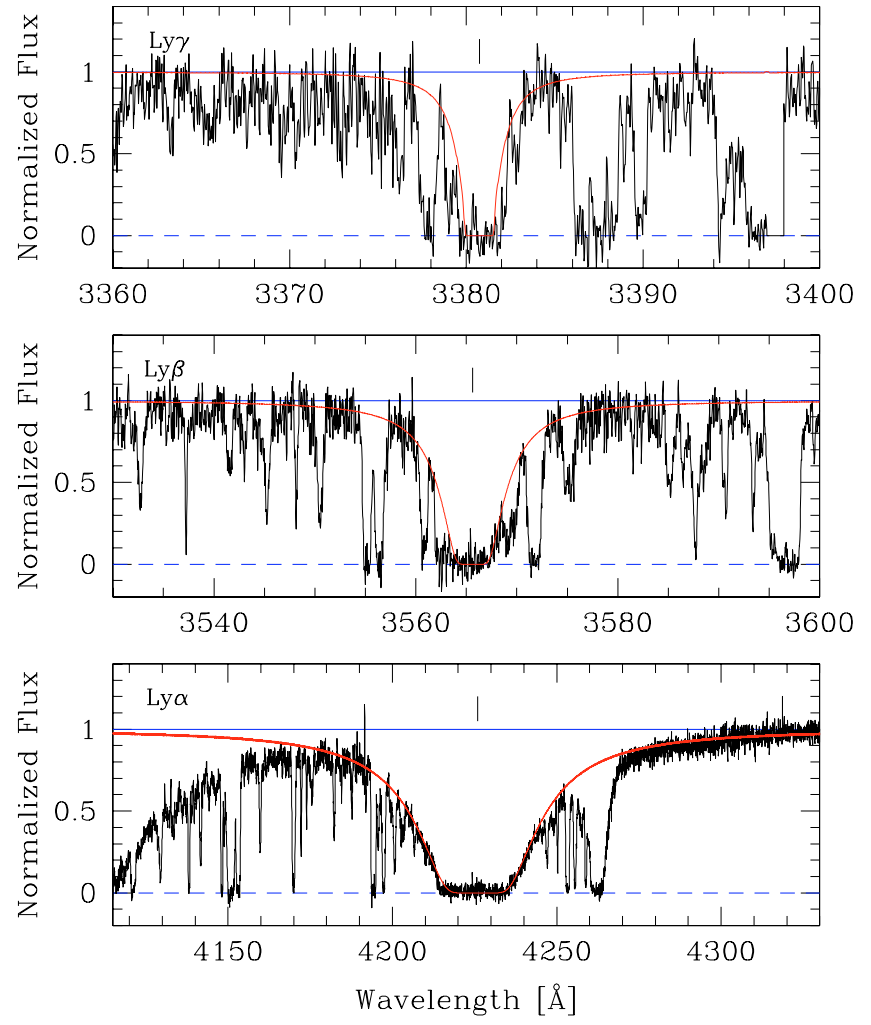

Fig. 5. Normalized UVES spectrum of Q $0841+129$ showing the $z_{\mathrm{abs}}=$ 2.476 DLA $\operatorname{Ly} \alpha, \operatorname{Ly} \beta$, and $\operatorname{Ly} \gamma$ line profiles with the Voigt profile fits. The vertical bar corresponds to the wavelength centroid of the component used for the best fit, $z=2.47621$. The measured H I column density is $\log N(\mathrm{HI})=20.78 \pm 0.08$. 
M. Dessauges-Zavadsky et al.: Data analysis and chemical variation studies, Online Material p 14
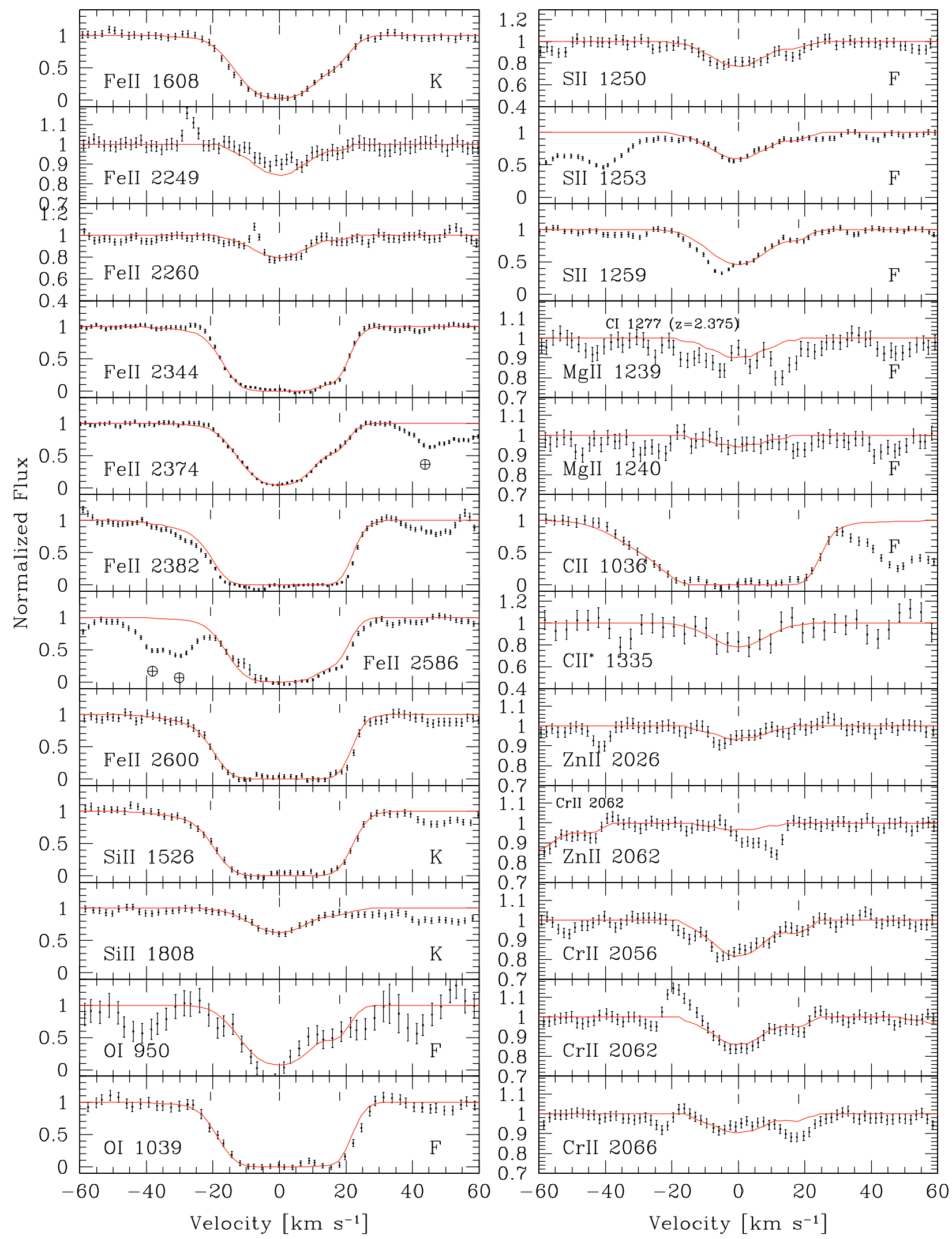

Fig. 6. Same as Fig. 2 for the second DLA toward Q $0841+129$ at $z_{\text {abs }}=2.476$. The zero velocity is fixed at $z=2.47621$. 
M. Dessauges-Zavadsky et al.: Data analysis and chemical variation studies, Online Material p 15
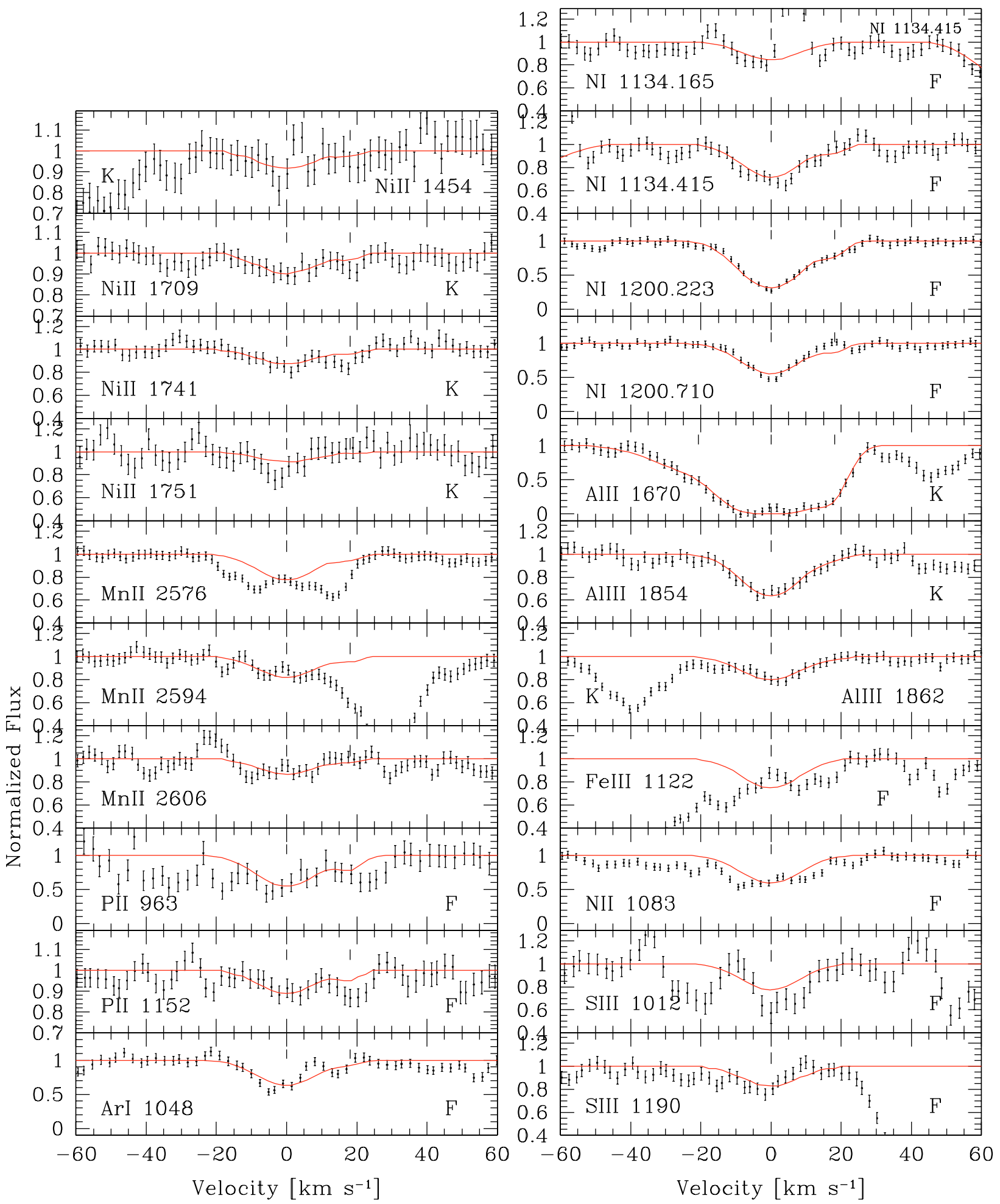

Fig. 6. continued. 
M. Dessauges-Zavadsky et al.: Data analysis and chemical variation studies, Online Material p 16

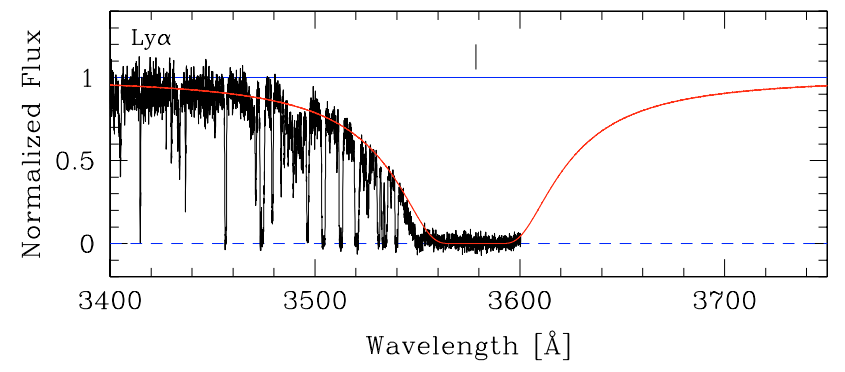

Fig. 7. Normalized UVES spectrum of Q1157+014 showing the DLA blue wing of the Ly $\alpha$ line profile with the Voigt profile fit. The vertical bar corresponds to the wavelength centroid of the component used for the best fit, $z=1.94366$. The measured H I column density is $\log N(\mathrm{HI})=21.60 \pm 0.10$. 
M. Dessauges-Zavadsky et al.: Data analysis and chemical variation studies, Online Material p 17
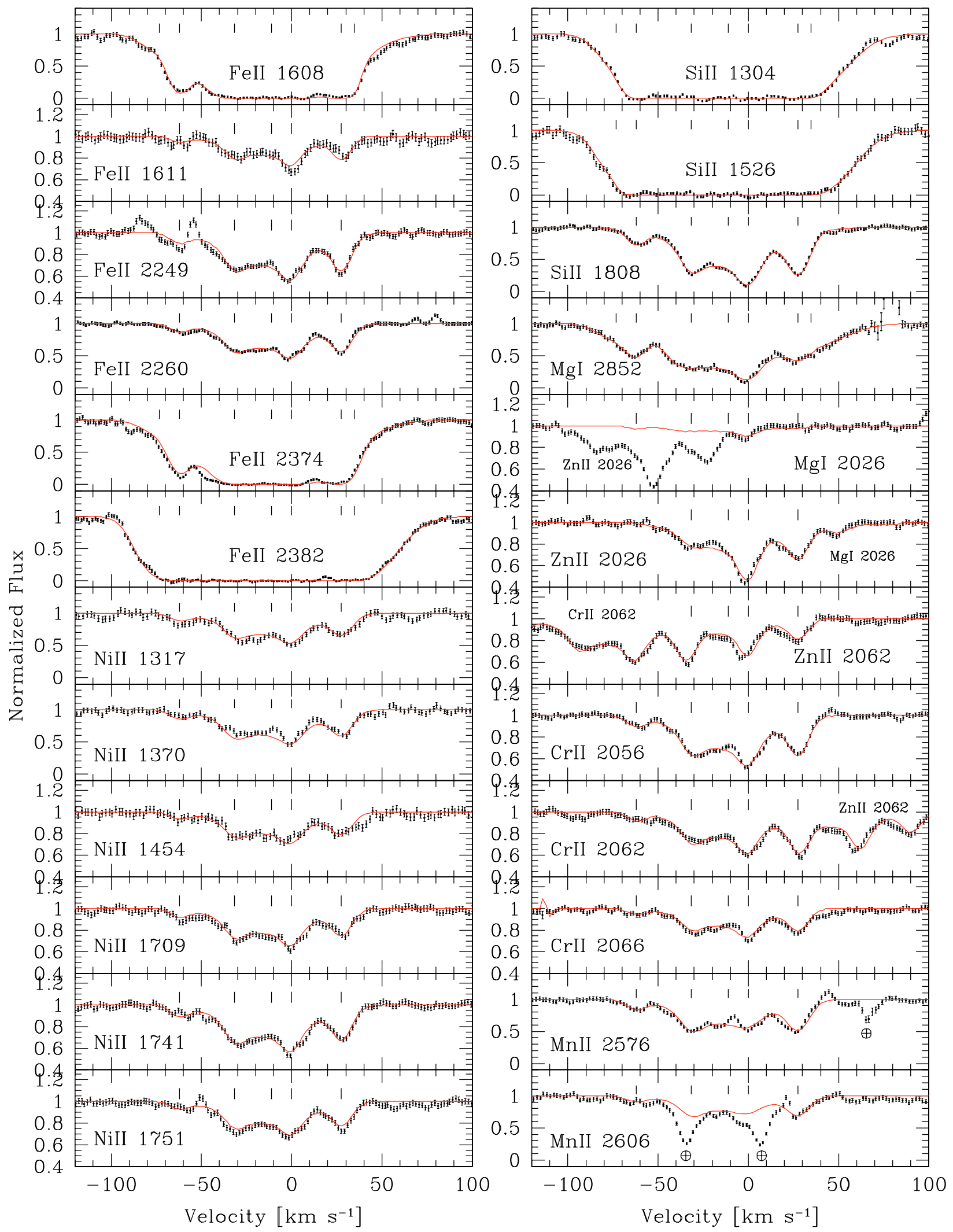

Fig. 8. Same as Fig. 2 for the DLA toward Q1157+014. The zero velocity is fixed at $z=1.94377$. 
M. Dessauges-Zavadsky et al.: Data analysis and chemical variation studies, Online Material p 18
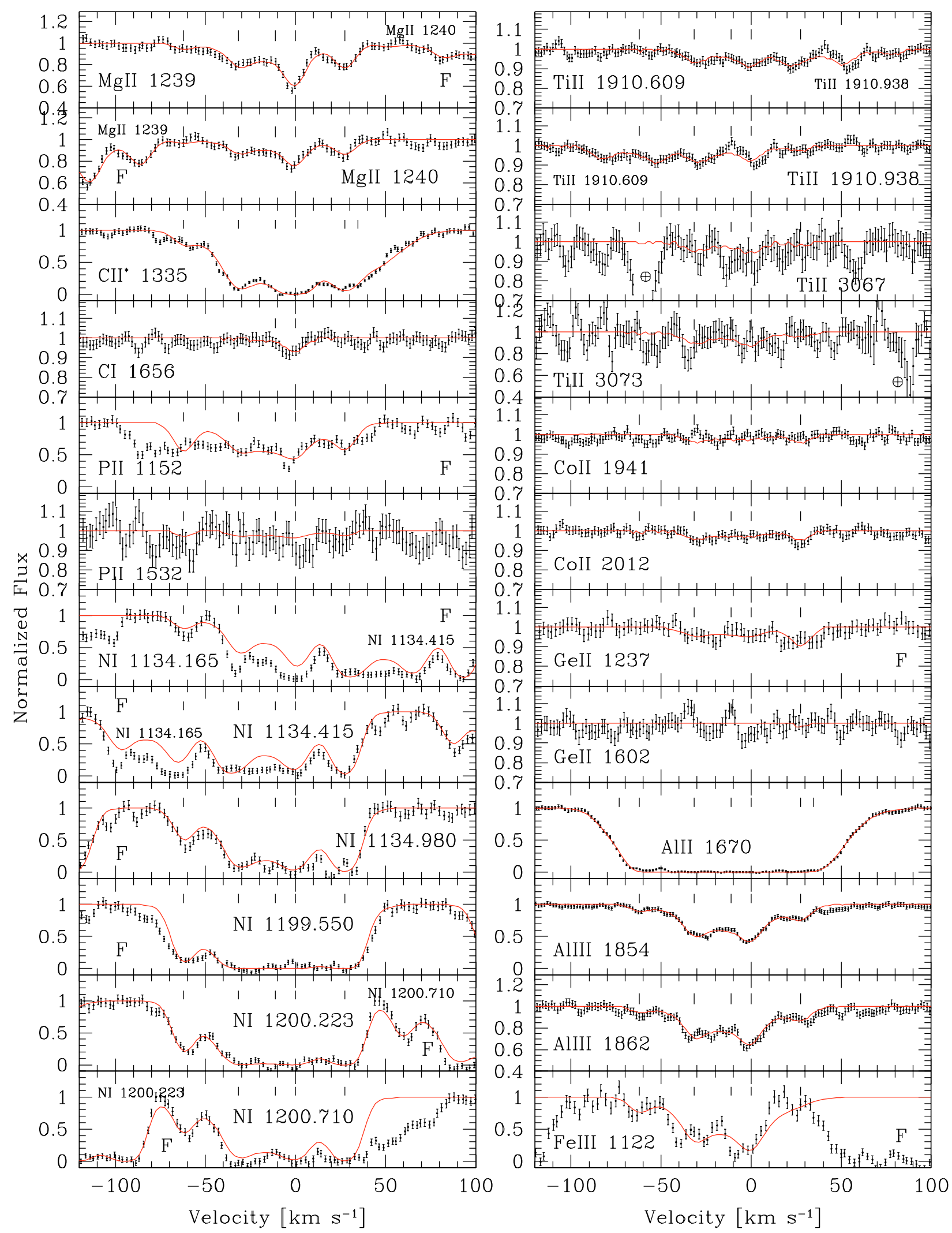

Fig. 8. continued. 
M. Dessauges-Zavadsky et al.: Data analysis and chemical variation studies, Online Material p 19

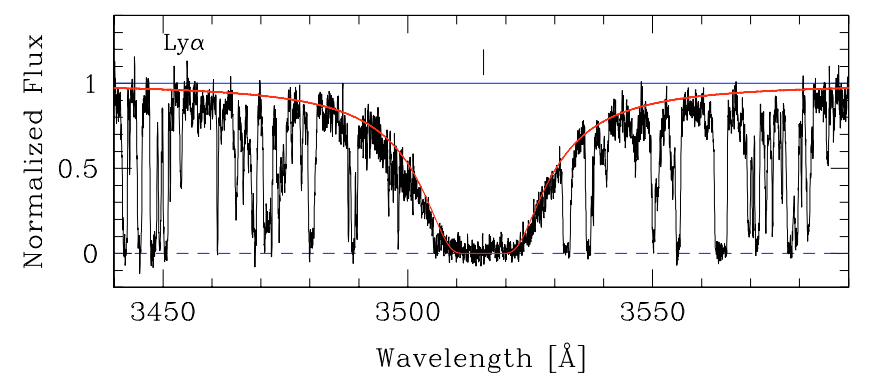

Fig. 9. Normalized UVES spectrum of Q1210+17 showing the DLA Ly $\alpha$ line profile with the Voigt profile fit. The vertical bar corresponds to the wavelength centroid of the component used for the best fit, $z=1.89177$. The measured HI column density is $\log N(\mathrm{H} \mathrm{I})=20.63 \pm 0.08$. 
M. Dessauges-Zavadsky et al.: Data analysis and chemical variation studies, Online Material p 20
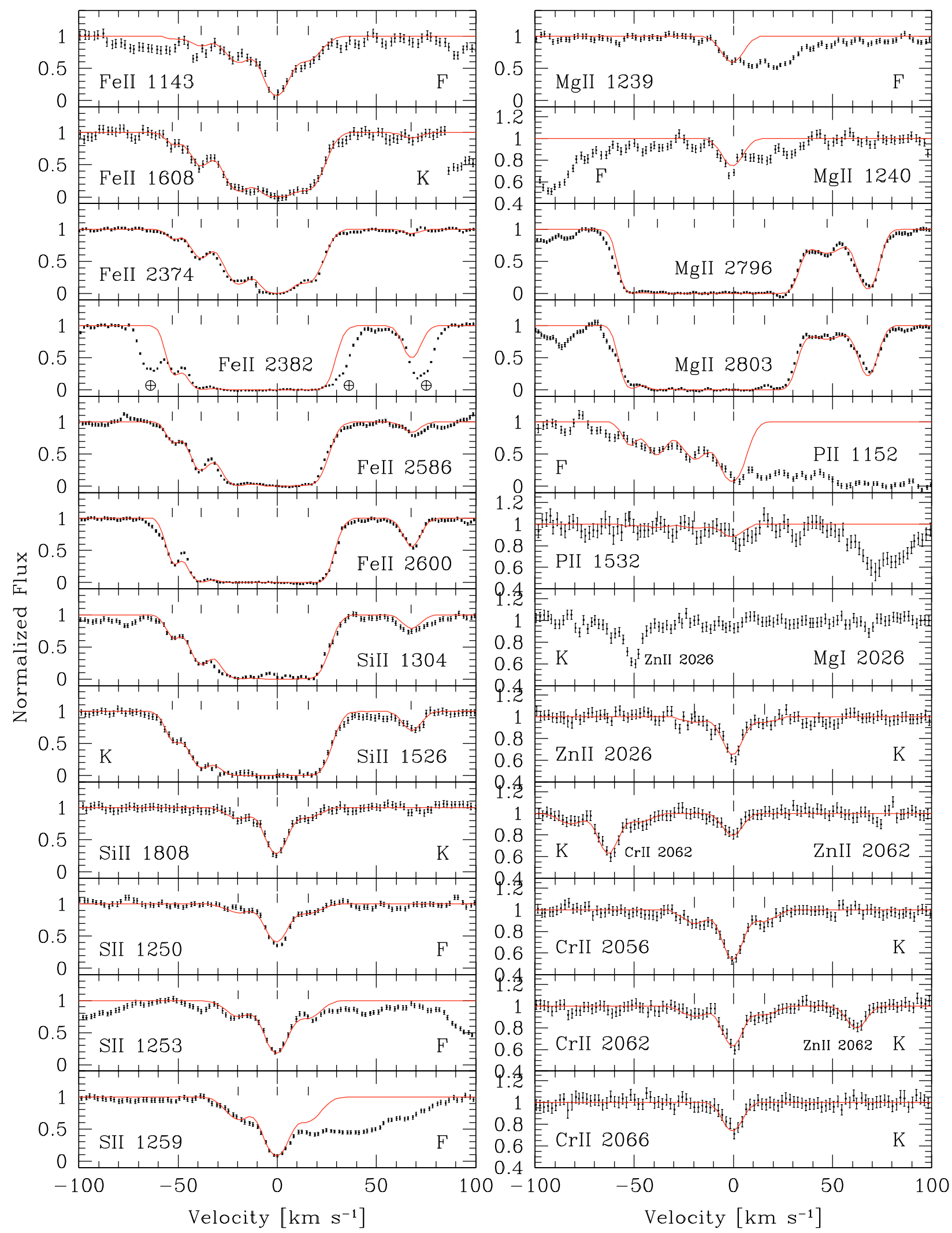

Fig. 10. Same as Fig. 2 for the DLA toward Q1210+17. The zero velocity is fixed at $z=1.89177$. 
M. Dessauges-Zavadsky et al.: Data analysis and chemical variation studies, Online Material p 21

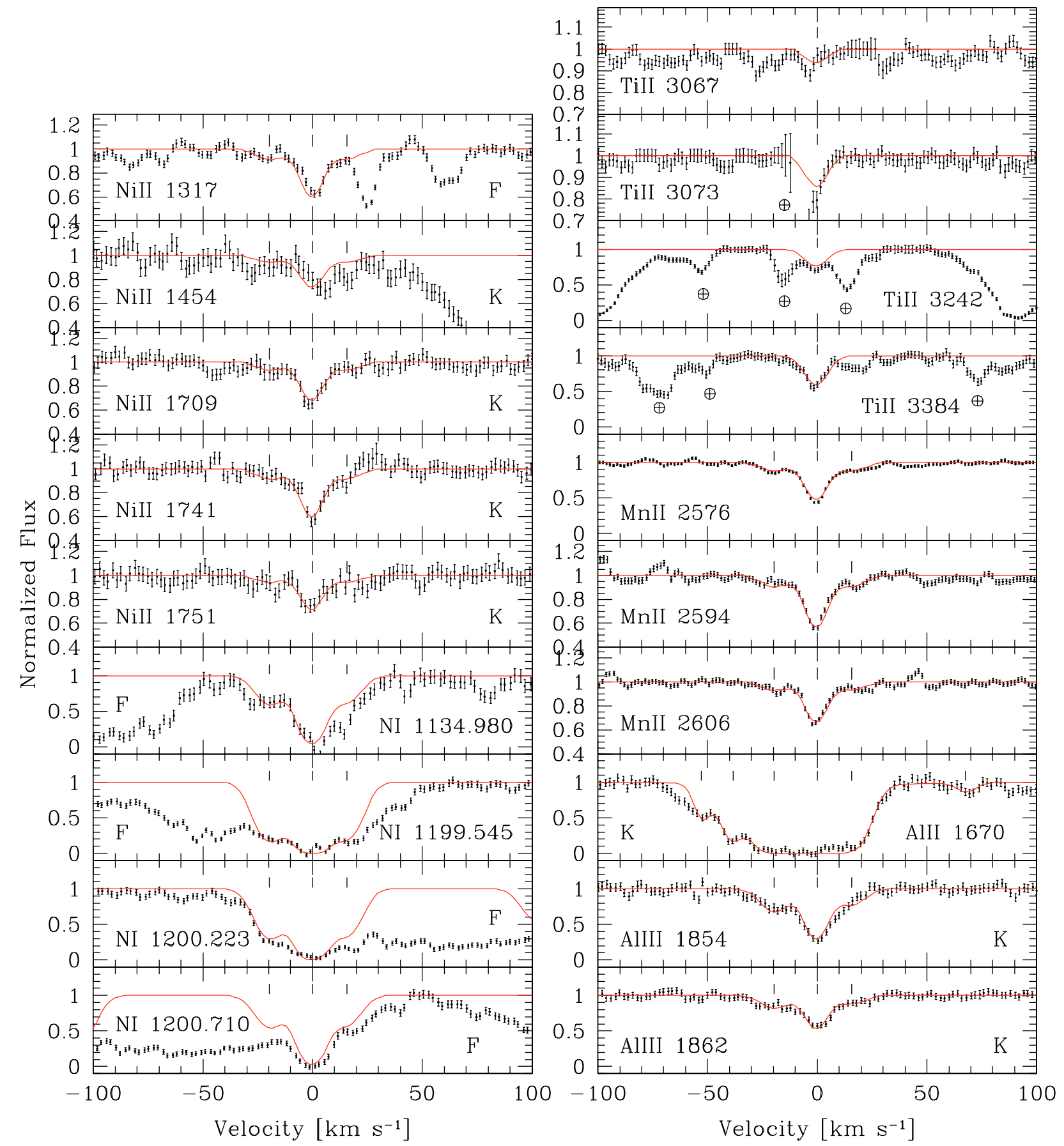

Fig. 10. continued. 
M. Dessauges-Zavadsky et al.: Data analysis and chemical variation studies, Online Material p 22

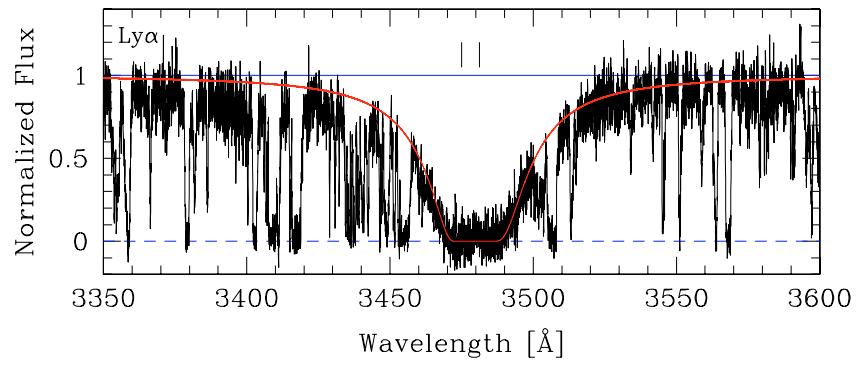

Fig. 11. Normalized UVES spectrum of Q2230+02 showing the DLA Ly $\alpha$ line profile with the Voigt profile fit. The vertical bars correspond to the wavelength centroids of the components used for the best fit, $z=1.86375$ and $z=1.85851$, referring to the DLA system and an additional absorber, respectively. The measured $\mathrm{H}$ I column densities are $\log N(\mathrm{HI})=20.83 \pm 0.05$ and $20.00 \pm 0.10$, respectively. 
M. Dessauges-Zavadsky et al.: Data analysis and chemical variation studies, Online Material p 23
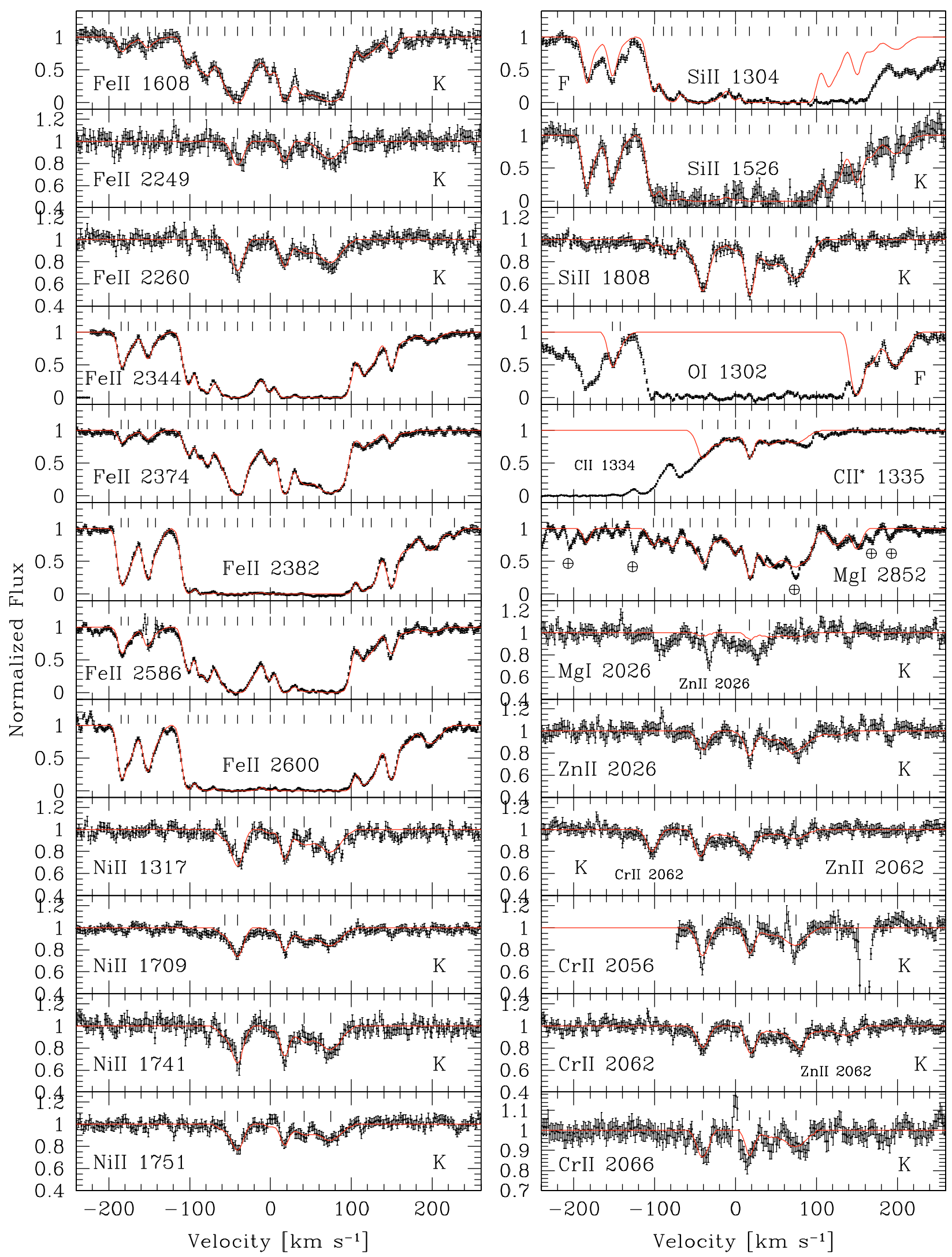

Fig. 12. Same as Fig. 2 for the DLA toward Q2230+02. The zero velocity is fixed at $z=1.86359$. 
M. Dessauges-Zavadsky et al.: Data analysis and chemical variation studies, Online Material p 24
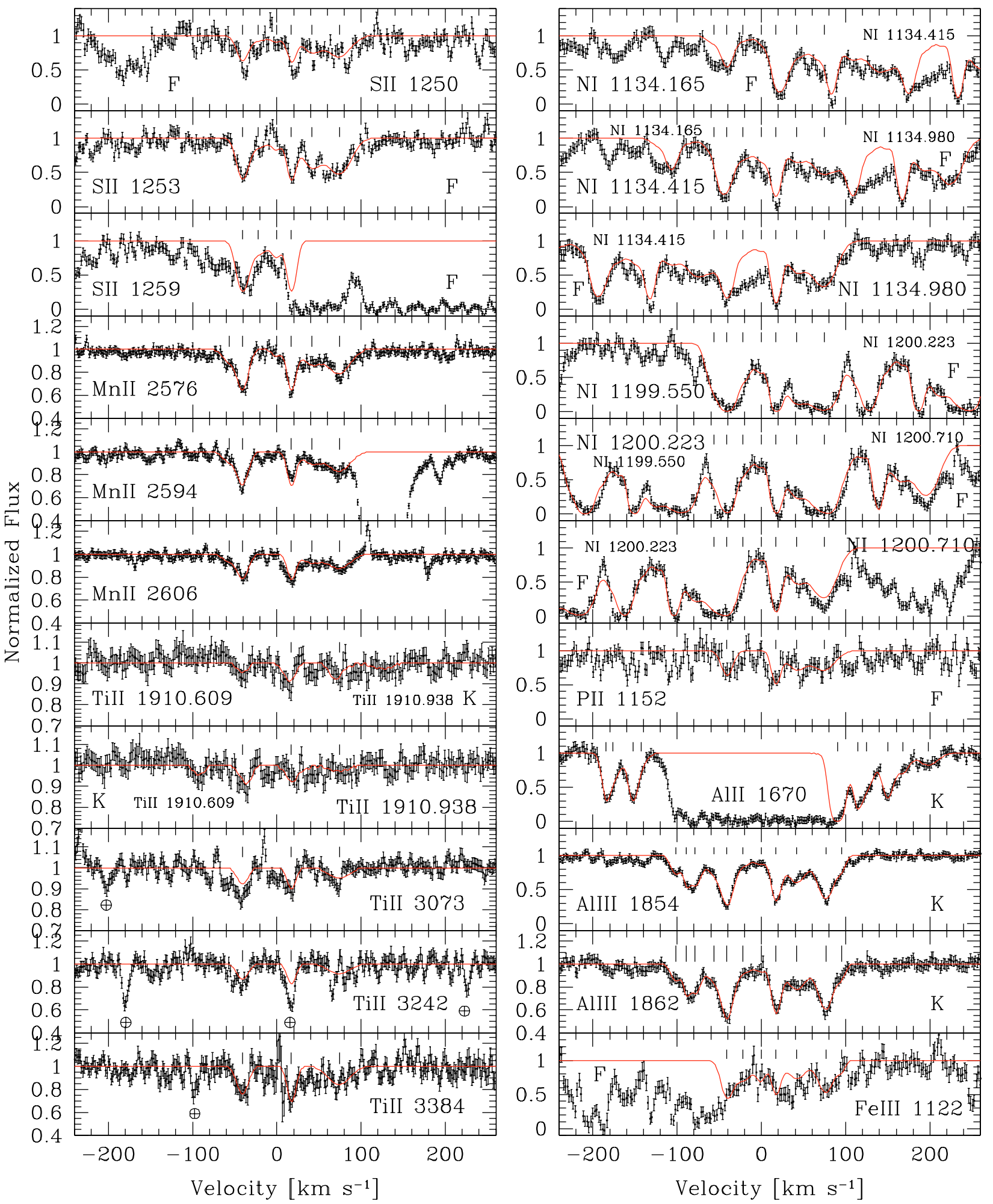

Fig. 12. continued. 
M. Dessauges-Zavadsky et al.: Data analysis and chemical variation studies, Online Material p 25

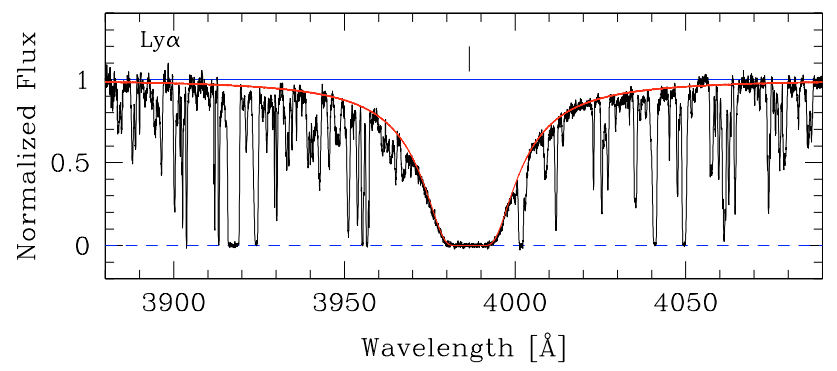

Fig. 13. Normalized UVES spectrum of Q2348-1444 showing the DLA Ly $\alpha$ line profile with the Voigt profile fit. The vertical bar corresponds to the wavelength centroid of the component used for the best fit, $z=2.27939$. The measured HI column density is $\log N(\mathrm{HI})=20.59 \pm 0.08$. 
M. Dessauges-Zavadsky et al.: Data analysis and chemical variation studies, Online Material p 26
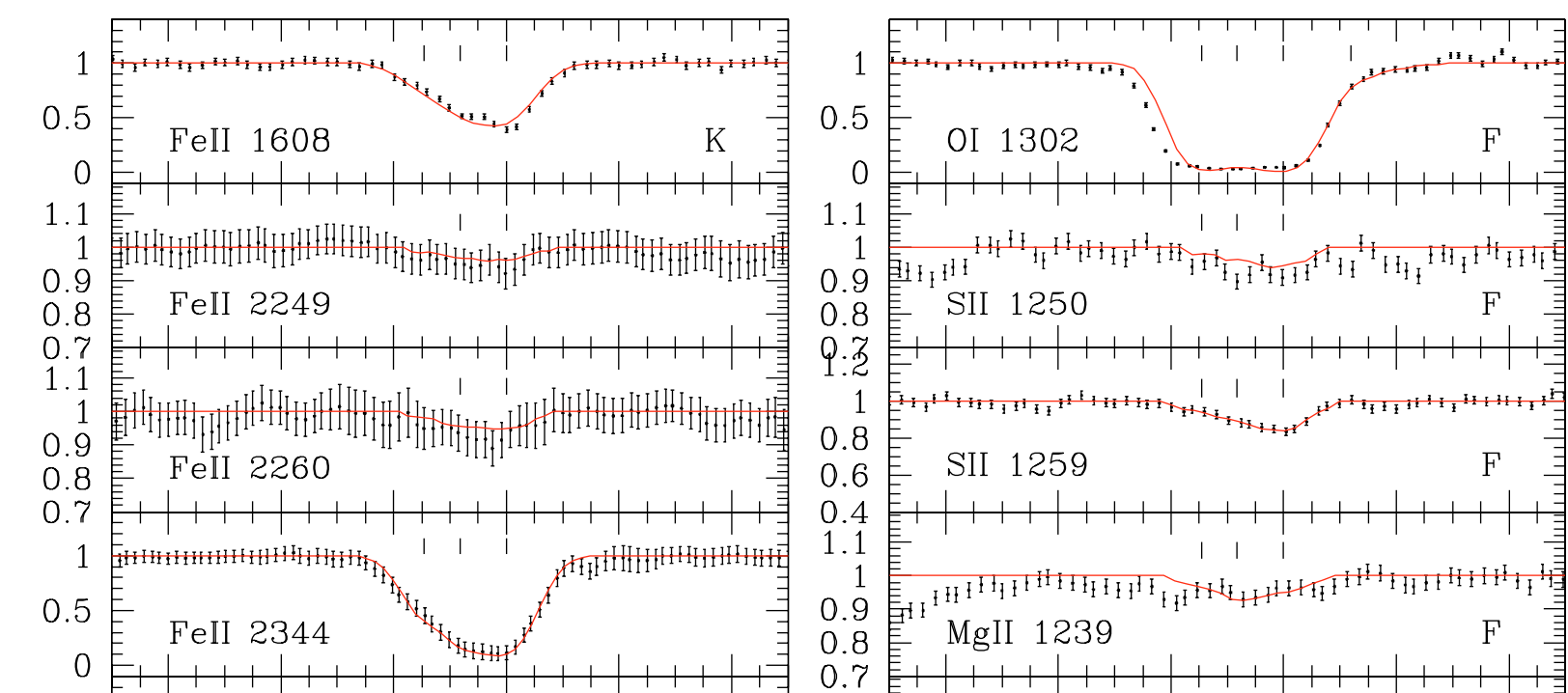

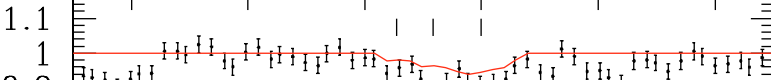

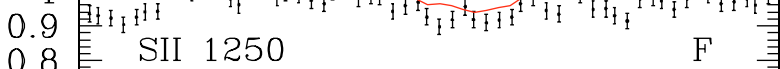

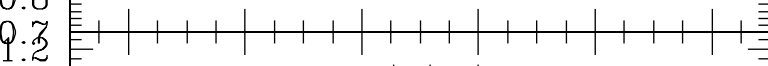

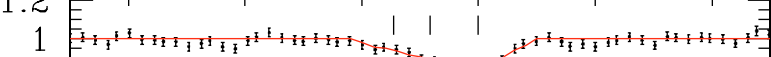

0.8 怎 SII 1259 F $\quad$ 毛

0.4 E E $+1+1+1+1+1+1+1+1+1+1|+|$

1.1 至 1 । ।

0.9 Fif $^{\mathrm{fH}}$ MgII 1239 F

0.7

1.

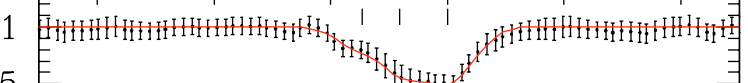

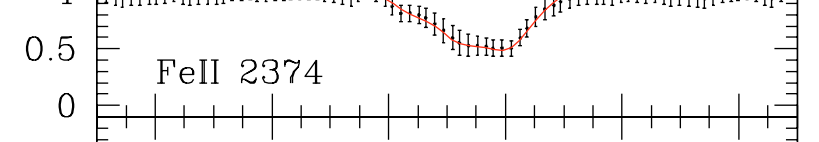

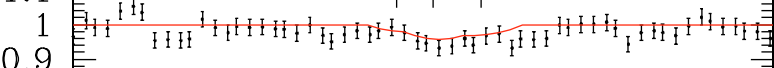

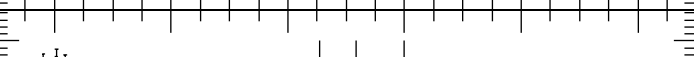

0.8 息 MgII $1240 \quad$ F

0.7

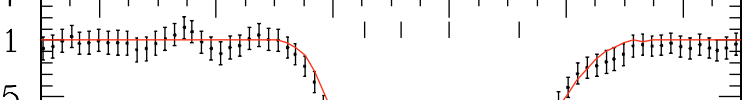

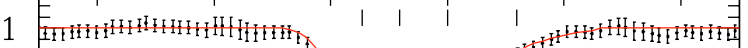

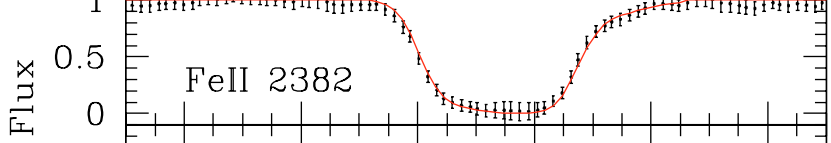

0.5

0

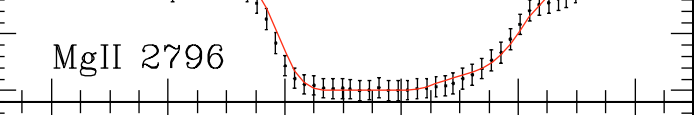

1

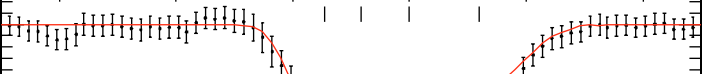

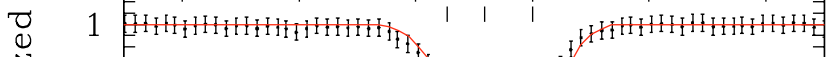
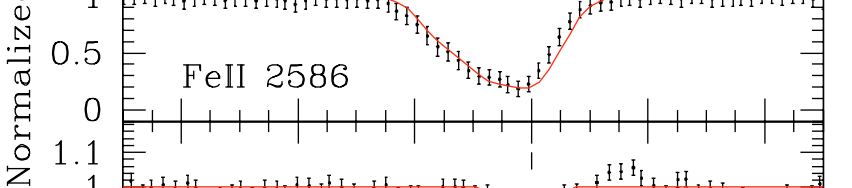

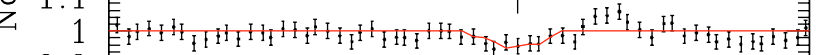

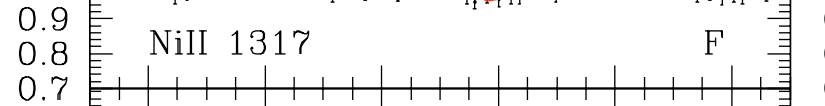

0.5

0

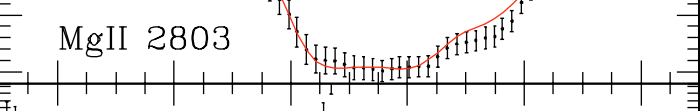

1.1 针

隹

0.9

.8

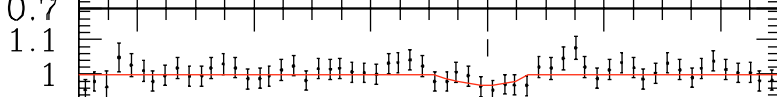

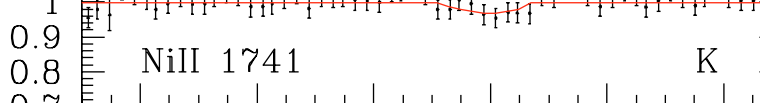

0.7

$+1++|+|+|+|+|+|+$

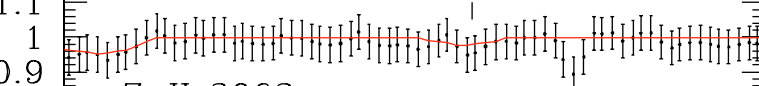

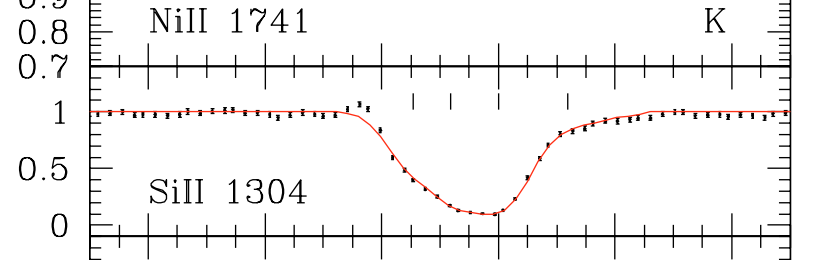

0.8 EnII 2062

0.7

1.1

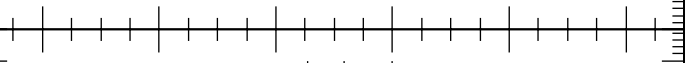

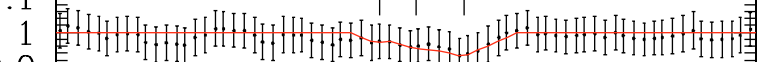

0.9 -

0.8 CrII 2056

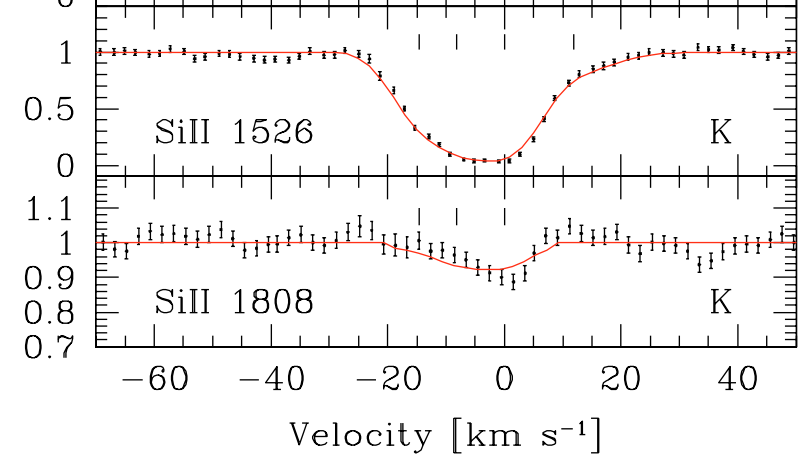

0.7

1.1

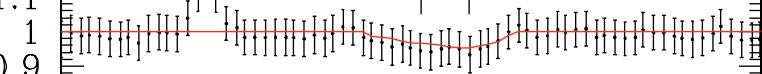

0.9 CrII 2062

0.8 E CrII 2062

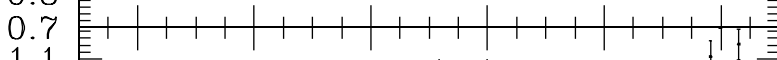

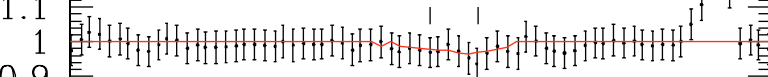

0.9 E $\quad$ CrII 2066

0.7

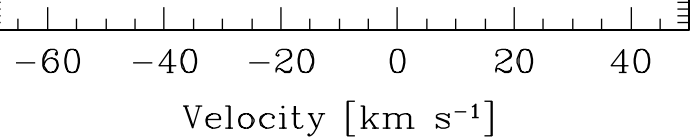

Fig. 14. Same as Fig. 2 for the DLA toward Q2348-1444. The zero velocity is fixed at $z=2.27939$. 
M. Dessauges-Zavadsky et al.: Data analysis and chemical variation studies, Online Material p 27
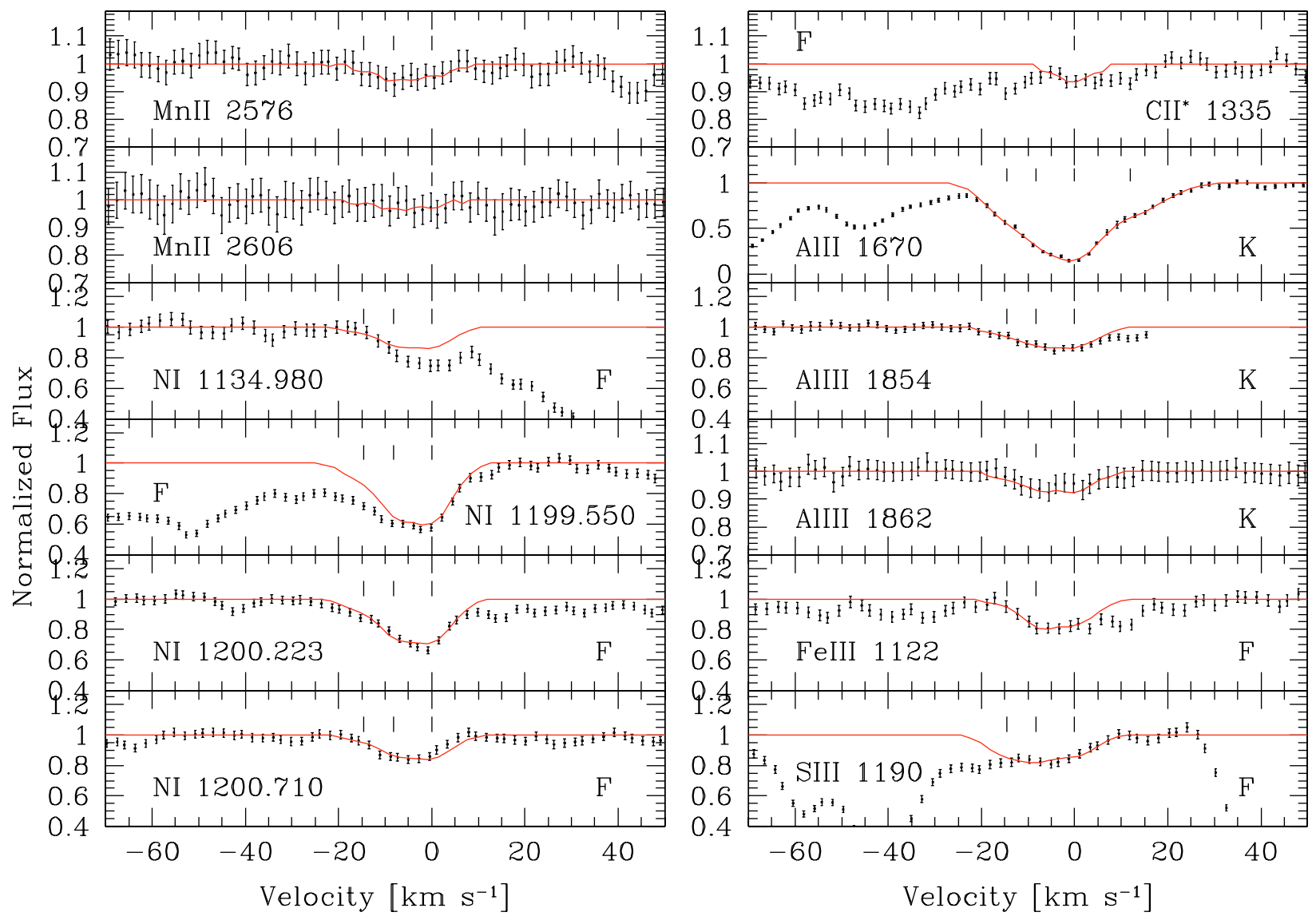

Fig. 14. continued. 


\section{Appendix A: An astrophysical determination of the Ni II $\lambda 1317$ oscillator strength}

In the analysis of ionic column densities of damped Ly $\alpha$ systems, we noticed that we derive a systematically lower $\mathrm{Ni}^{+}$column density measurement from the Ni II $\lambda 1317$ line than from the Ni II lines at $\lambda_{\text {rest }}=1454,1709,1741$, and $1751 \AA$, when all these lines are available in the same DLA system. The simplest way to explain this discrepancy is to call the reliability of the oscillator strength of the Ni II $\lambda 1317$ line into question. The most recent atomic data published for the Ni II transitions are summarized in Table A.1. In this table we see that Morton (1991) atomic data of the Ni II $\lambda 1454,1709,1741,1751$ lines have been updated by Fedchak et al. (2000), while no new measurement of the $f$-value exists for the Ni II $\lambda 1317$ line.

Our UVES/VLT spectra, combined with HIRES/Keck spectra, allowed us to observe the complete sample of Ni II UV transitions in several DLAs with a resolution and a signal-tonoise ratio sufficient for accurate analysis. We therefore decided to use these data to derive an astrophysical oscillator strength of the Ni II $\lambda 1317$ transition.

The adopted method to determine this $f$-value is relatively simple. We assumed that the component-to-component column densities derived from the Voigt profile fitting of the Ni II lines at $\lambda_{\text {rest }}=1454,1709,1741$, and $1751 \AA$ have to be the same as those derived from the Ni II $\lambda 1317$ line, and we varied the NiII $\lambda 1317 f$-value by requiring that this line yield the same component-to-component column densities as the other Ni II lines. More concretely, we determined the best value of the Ni II $\lambda 1317$ oscillator strength via a $\chi^{2}$ minimization of theoretical Voigt profile fits to the observed absorption Ni II $\lambda 1317$ line. We fixed the fitting parameters, the component-to-component $b$-values and redshifts, to the values of the best-fitting solution obtained for the low-ion lines, and the component-to-component $\mathrm{Ni}^{+}$column densities to the values obtained from the Voigt profile fitting of Ni II lines other than the Ni II $\lambda 1317$ line (see Tables 2-8).

We performed this analysis in five DLA systems, in which we detect, with high accuracy, at least two Ni II lines besides the NiII $\lambda 1317$ line. We do not consider the Ni II $\lambda 1370$ line in this analysis, since it also seems to show some discrepancy to the best-fit solution obtained from the Ni II $\lambda 1454,1709,1741,1751$ lines. The five selected DLAs are the DLA at $z_{\mathrm{abs}}=2.375$ toward Q0841+129, the DLA toward Q1157+014, the DLA toward Q1210+17, the DLA toward Q2230+02, and the DLA toward Q2231-00 studied in our first sample of DLAs (see Paper I). Their accessible Ni II lines, with their corresponding profiles, can be found in Figs. 4 , 8, 10, and 12, and Fig. 6 of Paper I, respectively.

The new Ni II $\lambda 1317$ oscillator strength results are illustrated in Fig. A.1 and summarized in Table A.2. Instead of showing the derived $f$-values through the best Voigt profile fitting solutions obtained for the Ni II lines, we present the results by plotting the apparent column density $N_{\mathrm{a}}(v)$ profiles for the analyzed Ni II lines. The apparent ionic column density for each pixel, $N_{\mathrm{a}}(v)$, is defined by:

$N_{\mathrm{a}}(v)=\frac{m_{\mathrm{e}} c}{\pi \mathrm{e}^{2}} \frac{\tau_{\mathrm{a}}(v)}{f \lambda}$,
Table A.1. Atomic data of the Ni II transitions.

\begin{tabular}{lccl}
\hline \hline Transition & $\lambda_{\text {rest }}$ & $f$ & Reference \\
\hline Ni II $\lambda 1317$ & 1317.2170 & 0.07786 & Morton (1991) \\
Ni II $\lambda 1370$ & 1370.1310 & 0.07690 & Fedchak \& Lawler (1999) \\
Ni II $\lambda 1454$ & 1454.8420 & 0.03230 & Fedchak et al. (2000) \\
Ni II $\lambda 1709$ & 1709.6042 & 0.03240 & Fedchak et al. (2000) \\
Ni II $\lambda 1741$ & 1741.5531 & 0.04270 & Fedchak et al. (2000) \\
Ni II $\lambda 1751$ & 1751.9156 & 0.02770 & Fedchak et al. (2000) \\
\hline
\end{tabular}

Table A.2. The new Ni II $\lambda 1317$ oscillator strength.

\begin{tabular}{lc}
\hline \hline DLA system & New $f$ \\
\hline$z_{\text {abs }}=2.375$ toward Q0841+129 & $0.052 \pm 0.010$ \\
$z_{\text {abs }}=1.944$ toward Q1157+014 & $0.064 \pm 0.007$ \\
$z_{\text {abs }}=1.892$ toward Q1210+17 & $0.052 \pm 0.007$ \\
$z_{\text {abs }}=1.864$ toward Q2230+02 & $0.054 \pm 0.006$ \\
$z_{\text {abs }}=2.066$ toward Q2231-00 & $0.064 \pm 0.008$ \\
\hline \multicolumn{2}{c}{$\left\langle f_{\text {new }}(\right.$ Ni II $\left.\lambda 1317)\right\rangle=0.057 \pm 0.006$} \\
\hline
\end{tabular}

$\dagger$ DLA studied in Paper I.

where $\tau_{\mathrm{a}}(v)=\ln \left[I_{\mathrm{i}}(v) / I_{\mathrm{a}}(v)\right], f$ is the oscillator strength, $\lambda$ the rest-wavelength, and $I_{\mathrm{i}}$ and $I_{\mathrm{a}}$ the incident intensity or, in other words, the continuum intensity $\left(I_{\mathrm{i}}(v)=1\right.$ in normalized spectra) and the measured intensity, respectively. For each of the five selected DLAs, we show the apparent column density $N_{\mathrm{a}}(v)$ profiles of the Ni II $\lambda 1709,1741,1751$ lines overplotted by a shaded profile (see Fig. A.1). This shaded profile corresponds to the Ni II $\lambda 1317$ apparent column density profile which was computed with the best result derived in each DLA individually for the new Ni II $\lambda 1317$ oscillator strength and its $1 \sigma$ error. We can observe that this shaded profile overlaps the apparent column density profiles of the Ni II lines at $\lambda_{\text {rest }}=1709,1741$, and $1751 \AA$ very well with exceptions for a few velocity components: the component at $v=-20 \mathrm{~km} \mathrm{~s}^{-1}$ in Fig. A.1 b), the components at $v=+42$ and $+74 \mathrm{~km} \mathrm{~s}^{-1}$ in Fig. A.1 d), and the component at $v=+11 \mathrm{~km} \mathrm{~s}^{-1}$ in Fig. A.1 e). The thick solid profile corresponds to the $\mathrm{Ni}$ II $\lambda 1317 N_{\mathrm{a}}(v)$ profile obtained with the old $f$-value from Morton (1991). It clearly shows the disagreement of this old $f$-value with the $N_{\mathrm{a}}(v)$ profiles of the Ni II $\lambda 1709,1741,1751$ lines.

The derived final new Ni II $\lambda 1317$ oscillator strength is:

$f_{\text {new }}($ Ni II $\lambda 1317)=0.057 \pm 0.006$.

It was obtained by computing a weighted mean of the five $f$-values derived in the five selected DLA systems (see Table A.2). The error corresponds to the rms dispersion of the five $f$-values. 
M. Dessauges-Zavadsky et al.: Data analysis and chemical variation studies, Online Material p 29
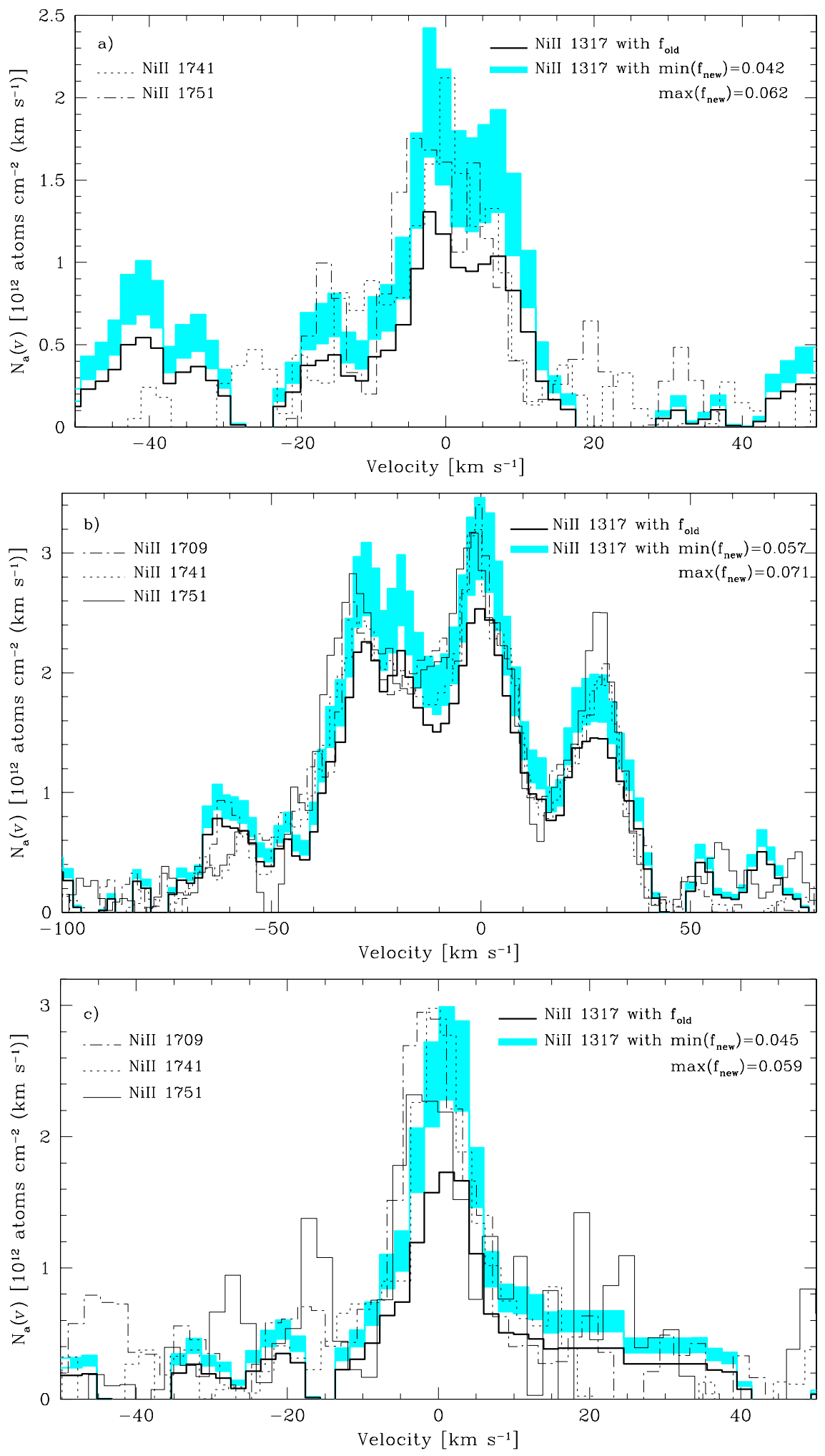

Fig. A.1. Apparent column density $N_{\mathrm{a}}(v)$ profiles for the Ni II $\lambda 1709,1741,1751$ lines (thin solid, dotted, and dashed-dotted lines). The shaded profile corresponds to the Ni II $\lambda 1317$ apparent column density profile computed with the best result derived in each DLA individually for the new Ni II $\lambda 1317$ oscillator strength and its $1 \sigma$ error. The thick solid profile corresponds to the Ni II $\lambda 1317 N_{\mathrm{a}}(v)$ profile obtained with the old $f$-value from Morton (1991). It is clearly lower than the apparent column density profiles for the Ni II $\lambda$ 1709, 1741, 1751 lines. The panels a), b), c), d), and e) correspond to the DLA at $z_{\text {abs }}=2.375$ toward Q0841+129, the DLA toward Q1157+014, the DLA toward Q1210+17, the DLA toward Q2230+02, and the DLA toward Q2231-00 studied in our first sample of DLAs (see Paper I), respectively. 
M. Dessauges-Zavadsky et al.: Data analysis and chemical variation studies, Online Material p 30
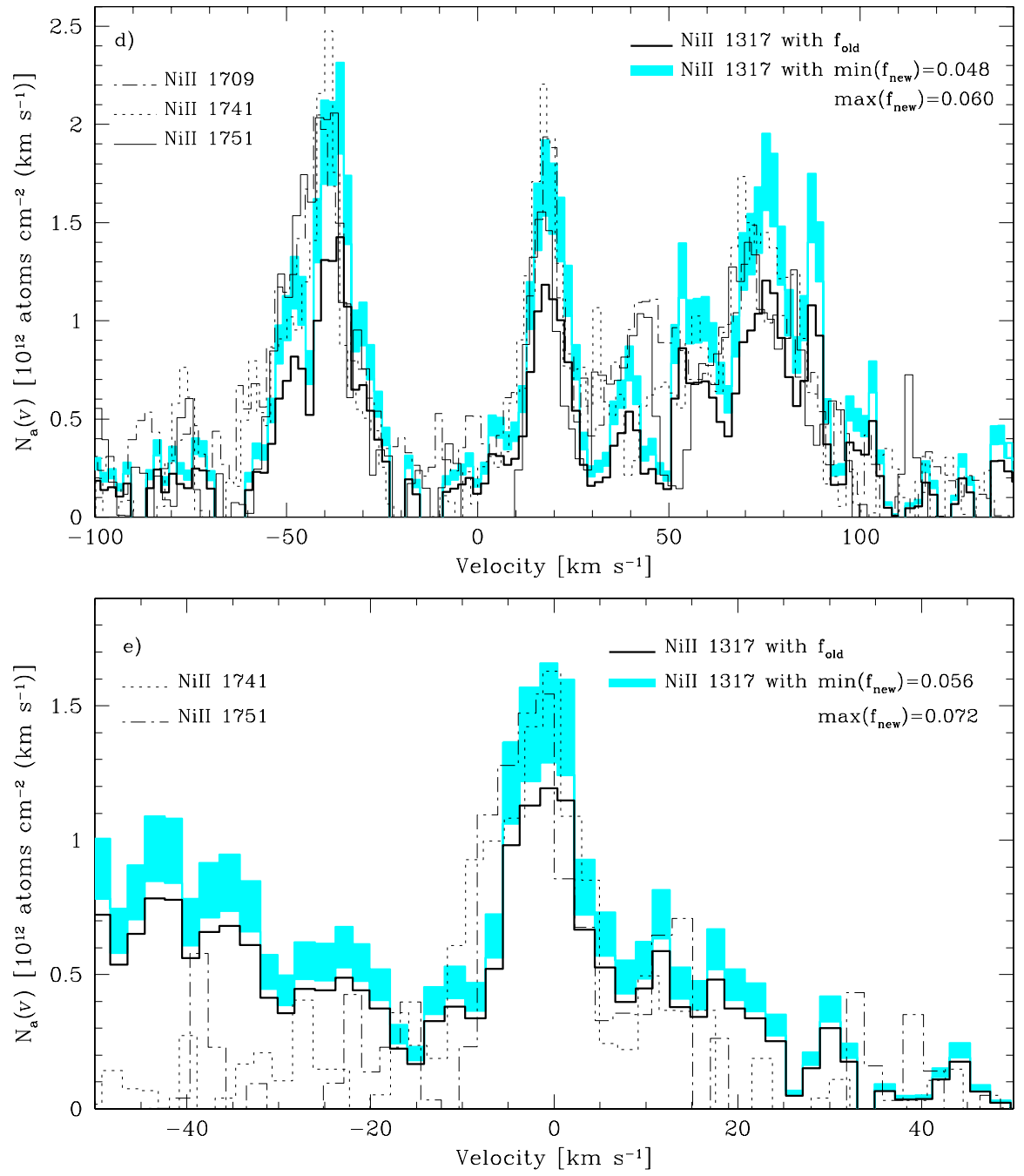

Fig. A.1. continued. 